\title{
Synthesis of 4'/5'-Spirocyclopropanated Uridine and D-Xylouridine Derivatives and their Activity against the Human Respiratory Syncytial Virus
}

\author{
Christoph Köllmann, Svenja M. Wiechert, Peter G. Jones, \\ Thomas Pietschmann, and Daniel B. Werz*
}

Supporting Information

Table of Contents

1. General Information

S2

2. Spectroscopic Data

S3

3. X-ray Structure Determinations

S61

4. Antiviral Investigations

S68

5. Conformational Investigations

S70

6. References

S71 


\section{General Information}

All solvents were purchased as synthetic grade solvents and stored over molecular sieves. All reactions were carried out in oven-dried glassware, septum-capped and under atmospheric pressure of argon. Commercially available compounds were used without further purification unless otherwise stated. Proton $\left({ }^{1} \mathrm{H}\right)$ and carbon $\left({ }^{13} \mathrm{C}\right)$ NMR spectra were recorded on a Bruker Avance II 300, a Bruker DRX 400, a Bruker Avance II 400 and a Bruker Avance II 600 spectrometer using the signals from tetramethylsilane as internal references for ${ }^{1} \mathrm{H}$ and ${ }^{13} \mathrm{C}$ chemical shifts, respectively. Abbreviations for multiplicities were used as following $s=$ singlet, $d$ = doublet, $\mathrm{t}=$ triplet, $\mathrm{q}=$ quartet, $\mathrm{p}=$ quintet, hept $=$ septet, $\mathrm{m}=$ multiplet. ESI-HRMS mass spectrometry was carried out on a FTICR instrument and IR spectra received from an ATR spectrometer. Optical rotations were measured on a Dr. Kernchen polarimeter at $20{ }^{\circ} \mathrm{C}$. HPLC separations were performed using an Agilent 1260 Infinity Series II system and an Agilent C18 column (Pursuit XRs 5 C18, 250.10.0 mm, flow rate at $4.50 \mathrm{ml} \cdot \mathrm{min}^{-1}$, detection at $254 \mathrm{~nm}$ ). 


\section{Spectroscopic Data}

\section{Compound 1}

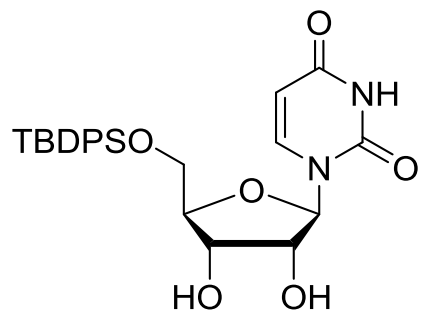

Compound 1 was prepared according to the procedure reported by van Otterlo et al. ${ }^{[1]}$

${ }^{1} \mathrm{H}$ NMR $\left(300 \mathrm{MHz}, \mathrm{CDCl}_{3}\right) \delta(\mathrm{ppm}) 7.98(\mathrm{~d}, J=8.1 \mathrm{~Hz}, 1 \mathrm{H}), 7.75-7.58(\mathrm{~m}, 4 \mathrm{H}), 7.50-7.33(\mathrm{~m}$, $6 \mathrm{H}), 5.94(\mathrm{~d}, J=2.7 \mathrm{~Hz}, 1 \mathrm{H}), 5.36(\mathrm{~d}, J=8.1 \mathrm{~Hz}, 1 \mathrm{H}), 4.43-4.34(\mathrm{~m}, 1 \mathrm{H}), 4.31$ (dd, $J=5.2,2.7$ $\mathrm{Hz}, 1 \mathrm{H}), 4.17-4.03(\mathrm{~m}, 2 \mathrm{H}), 3.95-3.81(\mathrm{~m}, 1 \mathrm{H}), 1.08(\mathrm{~s}, 9 \mathrm{H})$.

${ }^{13} \mathrm{C}$ NMR (151 MHz, $\left.\mathrm{CDCl}_{3}\right) \delta$ (ppm) 163.6, 150.9, 139.9, 135.4, 135.1, 132.6, 131.9, 129.9, 129.9, 127.8, 127.8, 102.1, 90.2, 84.4, 75.3, 68.9, 62.2, 26.8, 19.1. 


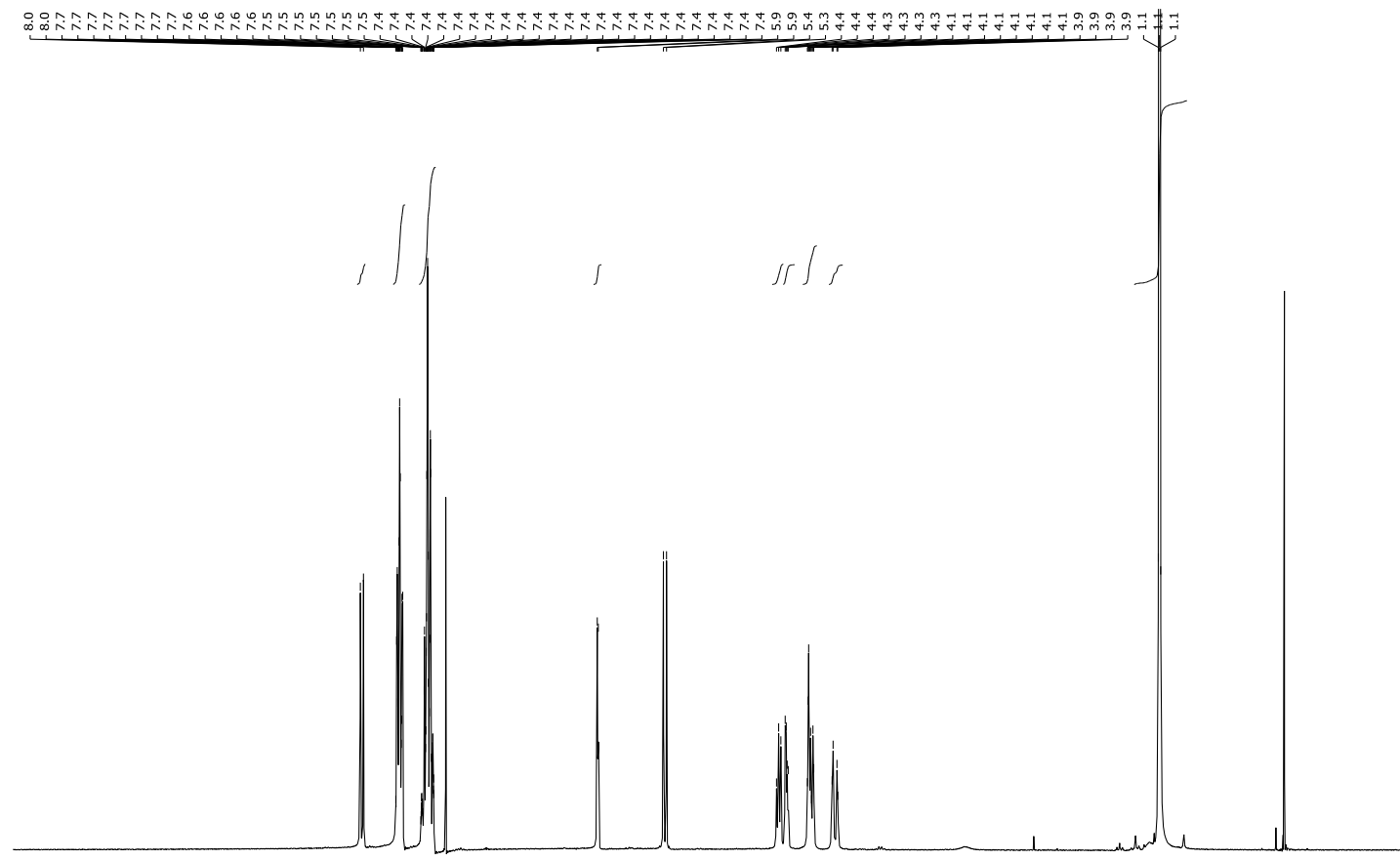

\begin{tabular}{lllllllllllllllllllllllllllll}
\hline & 1.0 & 10.5 & 10.0 & 9.5 & 9.0 & 8.5 & 8.0 & 7.5 & 7.0 & 6.5 & 6.0 & 5.5 & $\begin{array}{c}1 \\
\mathrm{f} 1(\mathrm{ppm})\end{array}$ & 4.5 & 4.0 & 3.5 & 3.0 & 2.5 & 2.0 & 1.5 & 1.0 & 0.5 & 0.0 & -0.5 & -1
\end{tabular}

Figure S1. ${ }^{1} \mathrm{H} \mathrm{NMR} \mathrm{(300} \mathrm{MHz,} \mathrm{CDCl}_{3}$ ) spectrum of compound 1.

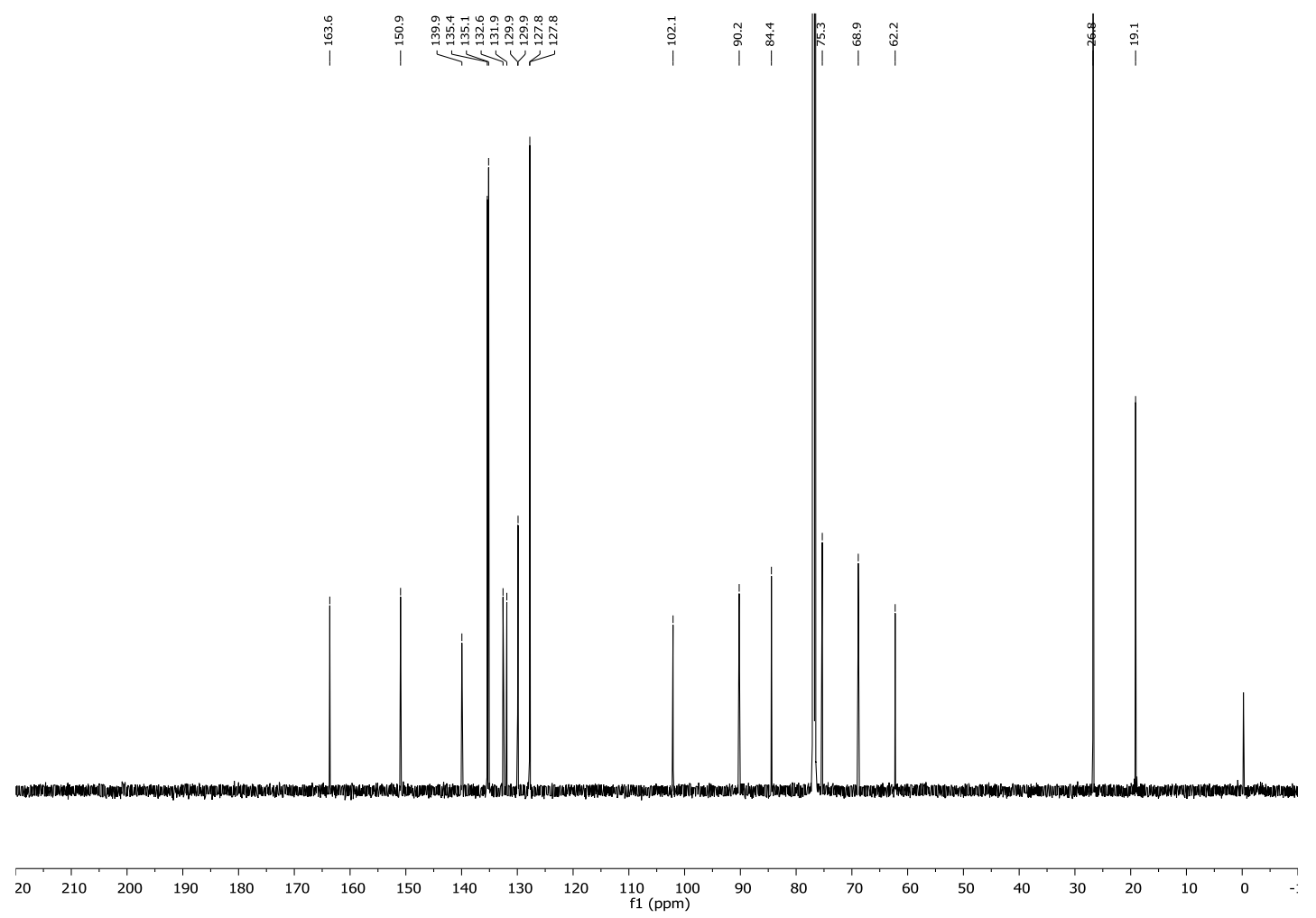

Figure S2. ${ }^{13} \mathrm{C}$ NMR $\left(151 \mathrm{MHz}, \mathrm{CDCl}_{3}\right)$ spectrum of compound 1. 


\section{Compound 2}

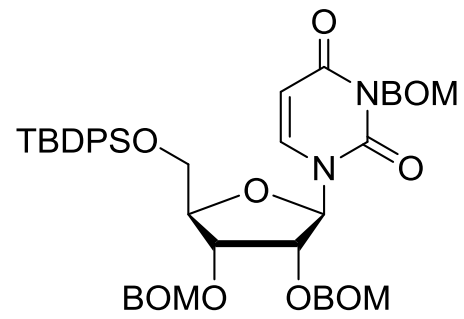

To a solution of compound 1 (6.77 g, $14.0 \mathrm{mmol}, 1.00$ eq.) in dichloromethane (100 mL) was successively added $\mathrm{NEt}^{\prime} \mathrm{Pr}_{2}(11.9 \mathrm{~mL}, 70.0 \mathrm{mmol}, 5.0$ eq.) and $\mathrm{BOMCl}(11.5 \mathrm{ml}, 84.0 \mathrm{mmol}$, 6.0 eq.) at $0{ }^{\circ} \mathrm{C}$. The mixture was stirred for $2 \mathrm{~d}$ at room temperature and finally one further equivalent of $\mathrm{BOMCl}$ was added (1.93 mL, $14.0 \mathrm{mmol}, 1.00$ eq.). After further stirring for $1 \mathrm{~d}$ and consumption of all remaining starting material, the mixture was diluted with dichloromethane $(200 \mathrm{~mL})$ and a saturated, aqueous solution of $\mathrm{NaHCO}_{3}(200 \mathrm{~mL})$. The aqueous layer was extracted with dichloromethane $(3 \mathrm{x}, 100 \mathrm{~mL})$, the combined organic phases dried over $\mathrm{Na}_{2} \mathrm{SO}_{4}$, filtered and evaporated to dryness. Purification by column chromatography $\left(\mathrm{SiO}_{2}\right.$, pentane/ethyl acetate, 10:1 $\rightarrow 1: 1)$ afforded the protected nucleoside 2 (9.92 $\mathrm{g}, 11.8 \mathrm{mmol}, 84 \%)$ as a yellowish oil.

${ }^{1} \mathrm{H}$ NMR $\left(600 \mathrm{MHz}, \mathrm{CDCl}_{3}\right) \delta(\mathrm{ppm}) 7.83(\mathrm{~d}, J=8.2 \mathrm{~Hz}, 1 \mathrm{H}), 7.69-7.64(\mathrm{~m}, 2 \mathrm{H}), 7.62-7.58(\mathrm{~m}$, $2 \mathrm{H}), 7.47-7.42(\mathrm{~m}, 2 \mathrm{H}), 7.41-7.33(\mathrm{~m}, 6 \mathrm{H}), 7.32-7.21(\mathrm{~m}, 13 \mathrm{H}), 6.09(\mathrm{~d}, J=3.0 \mathrm{~Hz}, 1 \mathrm{H}), 5.43$ $(\mathrm{d}, J=9.7 \mathrm{~Hz}, 1 \mathrm{H}), 5.40(\mathrm{~d}, J=9.7 \mathrm{~Hz}, 1 \mathrm{H}), 5.26(\mathrm{~d}, J=8.2 \mathrm{~Hz}, 1 \mathrm{H}), 5.02(\mathrm{~d}, J=6.9 \mathrm{~Hz}, 1 \mathrm{H})$, $4.91(\mathrm{~d}, J=6.8 \mathrm{~Hz}, 1 \mathrm{H}), 4.82(\mathrm{~d}, J=6.9 \mathrm{~Hz}, 1 \mathrm{H}), 4.80(\mathrm{~d}, J=6.9 \mathrm{~Hz}, 1 \mathrm{H}), 4.67$ (dd, $J=1.3,0.6$ $\mathrm{Hz}, 2 \mathrm{H}$ ), 4.65 (d, J=1.3 Hz, 2H), 4.59 (d, J=1.0 Hz, 2H), 4.38 (dd, J=6.7, $4.9 \mathrm{~Hz}, 1 \mathrm{H}$ ), 4.34 (dd, $J=4.9,3.0 \mathrm{~Hz}, 1 \mathrm{H}), 4.21(\mathrm{dt}, J=6.7,2.1 \mathrm{~Hz}, 1 \mathrm{H}), 4.13(\mathrm{dd}, J=12.0,2.1 \mathrm{~Hz}, 1 \mathrm{H}), 3.84(\mathrm{dd}, J=$ 12.1, $2.1 \mathrm{~Hz}, 1 \mathrm{H}), 1.09(\mathrm{~s}, 9 \mathrm{H})$.

${ }^{13} \mathrm{C} \mathrm{NMR}\left(151 \mathrm{MHz}, \mathrm{CDCl}_{3}\right) \delta$ (ppm) 162.5, 150.9, 138.3, 137.9, 137.4, 137.3, 135.6, 135.3, 132.7, 132.0, 130.2, 130.1, 128.5, 128.4, 128.3, 128.1, 128.0, 127.9, 127.7, 127.7, 127.6, 127.6, 127.6, 101.8, 94.4, 94.1, 88.6, 82.5, 78.6, 72.8, 72.2, 70.2, 70.0, 69.9, 62.4, 27.0, 19.4 .

IR (ATR): $\tilde{v}\left(\mathrm{~cm}^{-1}\right)=3067,3030,2956,2932,2895,2561,1715,1664,1495,1453,1428,1357$, 1274, 1216, 1171, 1108, 1066, 1024.

HR-MS (ESI): $m / z$ calcd for $\mathrm{C}_{49} \mathrm{H}_{54} \mathrm{~N}_{2} \mathrm{O}_{9} \mathrm{Na}^{+}:$865.34908; found: 865.34920 .

$[\alpha]_{\mathrm{D}}^{20}=440.3^{\circ}\left(\mathrm{c}=4.5, \mathrm{CHCl}_{3}\right)$ 


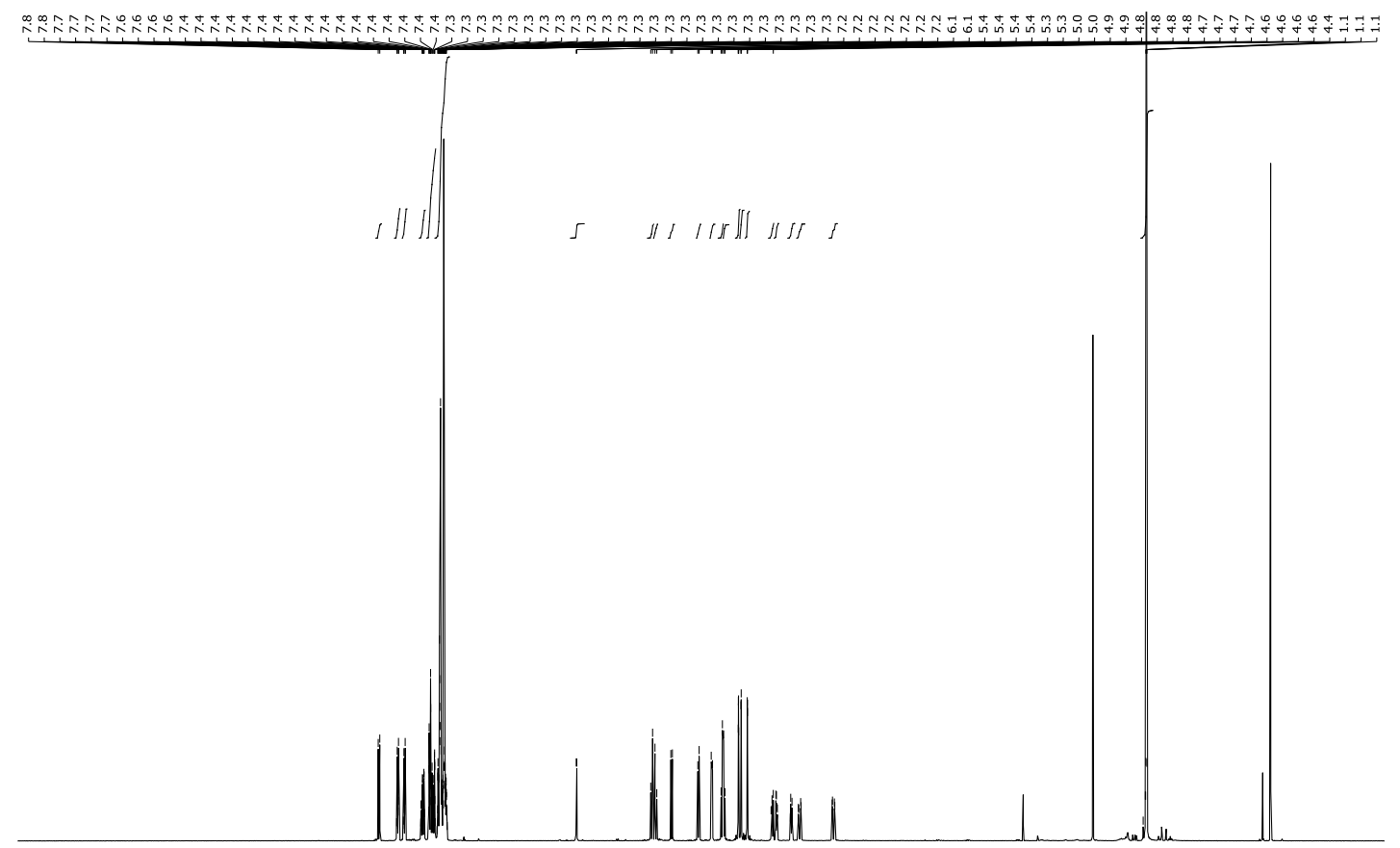

\begin{tabular}{lllllllllllllllllllllllllllll}
\hline & 1.0 & 10.5 & 10.0 & 9.5 & 9.0 & 8.5 & 8.0 & 7.5 & 7.0 & 6.5 & 6.0 & 5.5 & $\begin{array}{c}1 \\
\mathrm{f} 1(\mathrm{ppm})\end{array}$ & 4.5 & 4.0 & 3.5 & 3.0 & 2.5 & 2.0 & 1.5 & 1.0 & 0.5 & 0.0 & -0.5 & -1
\end{tabular}

Figure S3. ${ }^{1} \mathrm{H} \mathrm{NMR}\left(600 \mathrm{MHz}, \mathrm{CDCl}_{3}\right)$ spectrum of compound 2.

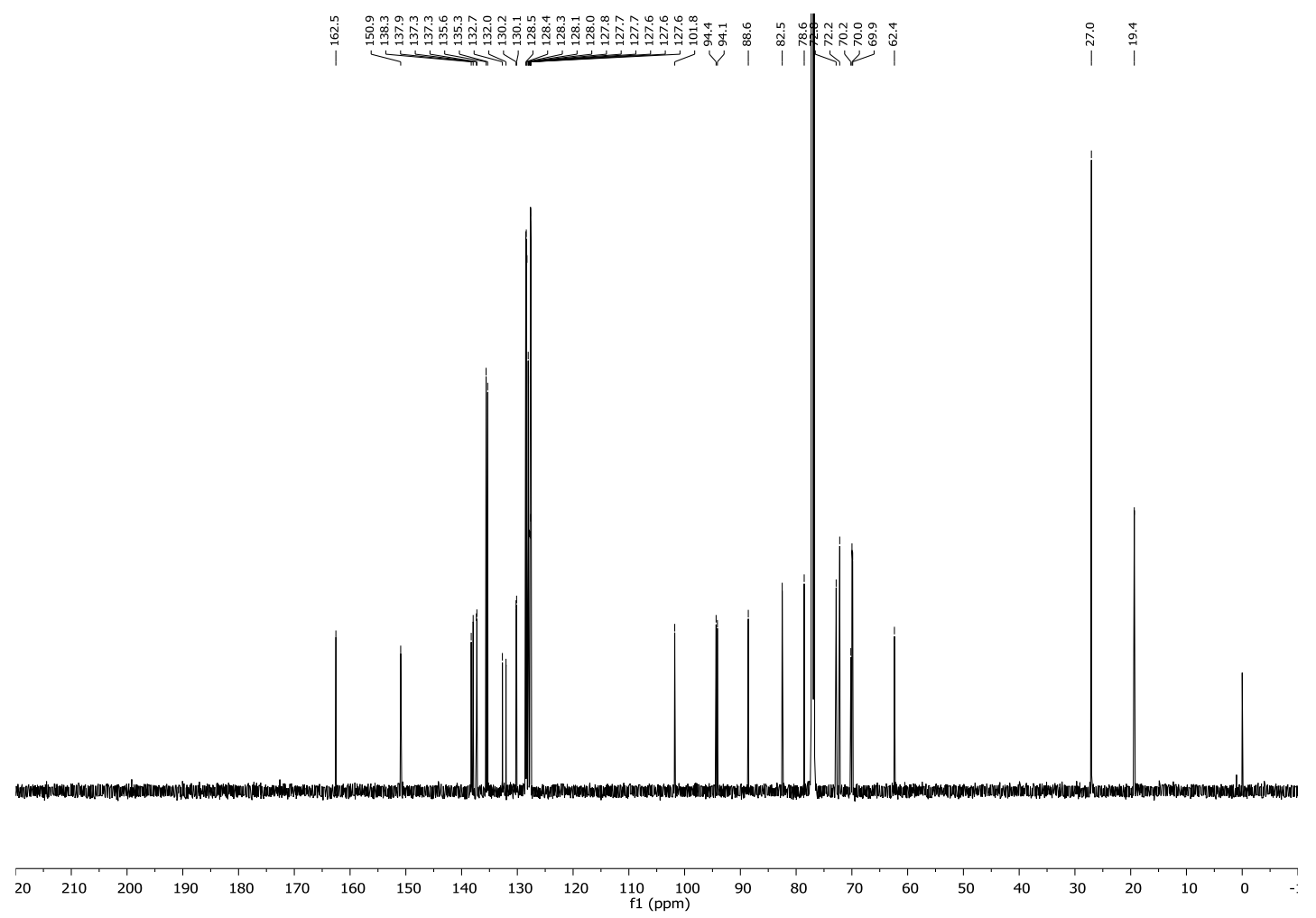

Figure S4. ${ }^{13} \mathrm{C}$ NMR $\left(151 \mathrm{MHz}, \mathrm{CDCl}_{3}\right)$ spectrum of compound 2. 


\section{Compound 3}

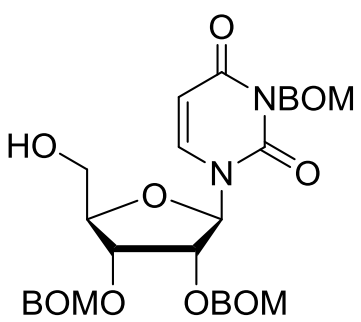

Silylated compound 2 (12.5 g, $14.8 \mathrm{mmol}, 1.0$ eq.) was dissolved in THF (100 mL) and TBAF $3 \mathrm{H}_{2} \mathrm{O}(7.00 \mathrm{~g}, 22.2 \mathrm{mmol}, 1.50$ eq.) was added at room temperature. The reaction mixture was stirred for $1 \mathrm{~h}$, diluted with ethyl acetate $(100 \mathrm{~mL})$ and the reaction terminated by the addition of a saturated $\mathrm{NaHCO}_{3}$ solution $(100 \mathrm{~mL})$. The aqueous layer was extracted with ethyl acetate $(3 \mathrm{x}, 100 \mathrm{~mL})$. The combined organic phases were washed with a saturated $\mathrm{NaCl}$ solution $(100 \mathrm{~mL})$, dried over $\mathrm{Na}_{2} \mathrm{SO}_{4}$, filtered and evaporated to dryness. Purification by column chromatography (neutralised $\mathrm{SiO}_{2}$, pentane/ethyl acetate, 1:1) afforded the primary alcohol 3 (7.76 g, $12.8 \mathrm{mmol}, 87 \%$ yield) as a colorless syrup.

${ }^{1} \mathbf{H}$ NMR $\left(400 \mathrm{MHz}, \mathrm{CDCl}_{3}\right) \delta(\mathrm{ppm}) 7.63(\mathrm{~d}, J=8.2 \mathrm{~Hz}, 1 \mathrm{H}), 7.42-7.18(\mathrm{~m}, 15 \mathrm{H}), 5.82(\mathrm{~d}, J=$ $3.5 \mathrm{~Hz}, 1 \mathrm{H}), 5.66(\mathrm{~d}, J=8.1 \mathrm{~Hz}, 1 \mathrm{H}), 5.49-5.30(\mathrm{~m}, 2 \mathrm{H}), 4.98(\mathrm{~d}, J=6.8 \mathrm{~Hz}, 1 \mathrm{H}), 4.91-4.78$ $(\mathrm{m}, 3 \mathrm{H}), 4.71-4.59(\mathrm{~m}, 6 \mathrm{H}), 4.50(\mathrm{dd}, J=5.2,3.5 \mathrm{~Hz}, 1 \mathrm{H}), 4.36(\mathrm{dd}, J=6.2,5.2 \mathrm{~Hz}, 1 \mathrm{H}), 4.23$ (dt, $J=6.2,2.2 \mathrm{~Hz}, 1 \mathrm{H}$ ), 3.98 (ddd, $J=12.3,4.2,2.1 \mathrm{~Hz}, 1 \mathrm{H}$ ), 3.76 (ddd, $J=12.3,6.0,2.3 \mathrm{~Hz}$, $1 \mathrm{H}), 2.53(\mathrm{dd}, J=6.0,4.2 \mathrm{~Hz}, 1 \mathrm{H})$.

${ }^{13} \mathrm{C}$ NMR (101 MHz, $\left.\mathrm{CDCl}_{3}\right) \delta$ (ppm) 162.5, 150.9, 139.8, 137.8, 137.2, 128.5, 128.4, 128.3, 128.0, 127.8, 127.7, 127.6, 127.5, 101.8, 94.7, 94.5, 91.4, 83.0, 78.0, 73.4, 72.2, 70.2, 70.2, 69.9, 61.1. IR (ATR): $\tilde{v}\left(\mathrm{~cm}^{-1}\right)=3475,3090,3064,3030,2940,2896,1712,1659,1496,1454,1411,1357$, 1273, 1214, 1170, 1064, 1024.

HR-MS (ESI): $m / z$ calcd for $\mathrm{C}_{33} \mathrm{H}_{36} \mathrm{~N}_{2} \mathrm{O}_{9} \mathrm{Na}^{+}:$627.23130; found: 627.23132.

$[\alpha]_{\mathrm{D}}^{20}=50.4^{\circ}\left(\mathrm{c}=0.76, \mathrm{CHCl}_{3}\right)$. 


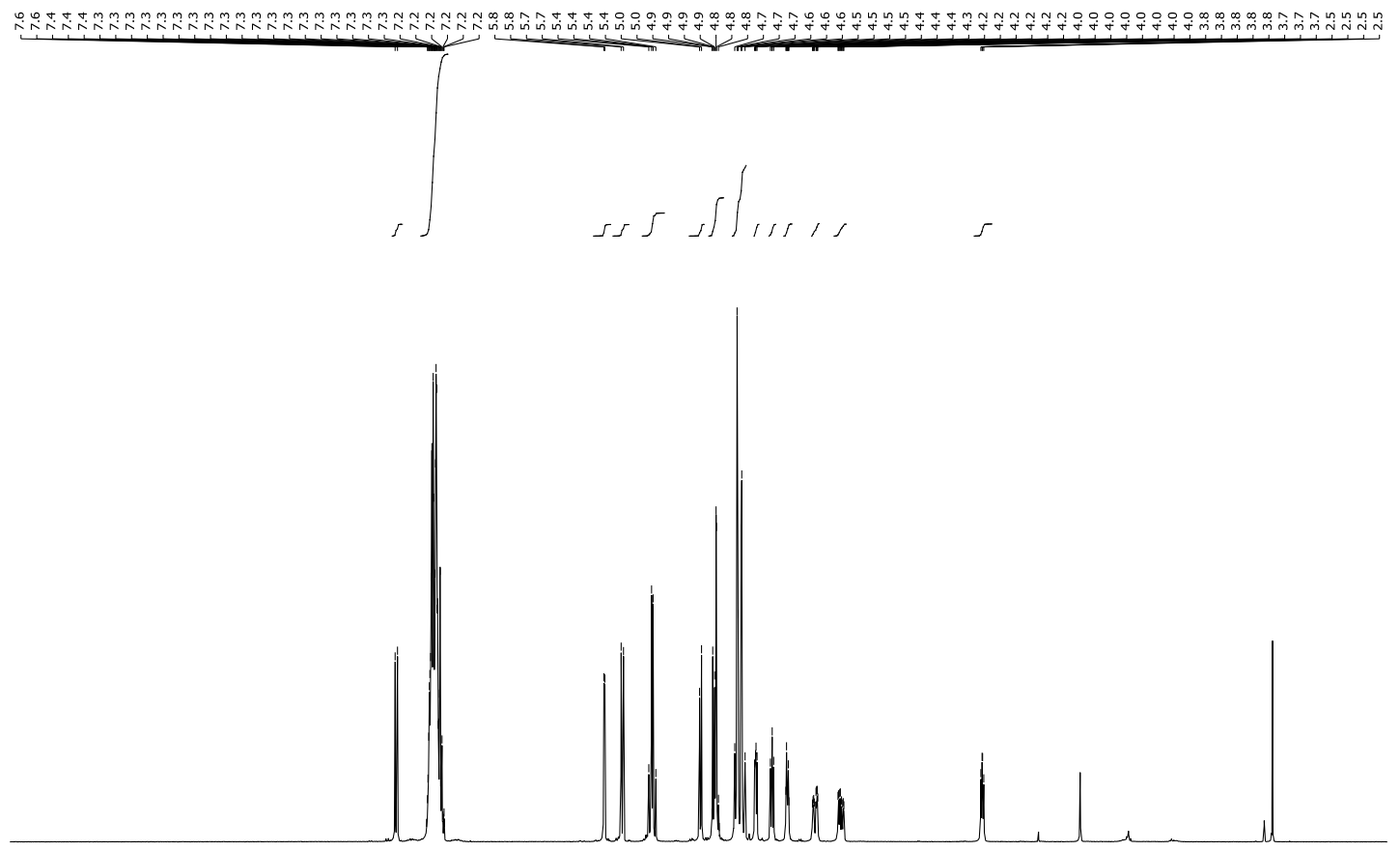

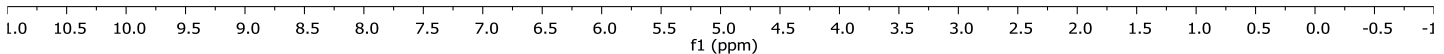

Figure S5. ${ }^{1} \mathrm{H}$ NMR $\left(400 \mathrm{MHz}, \mathrm{CDCl}_{3}\right)$ spectrum of compound 3.

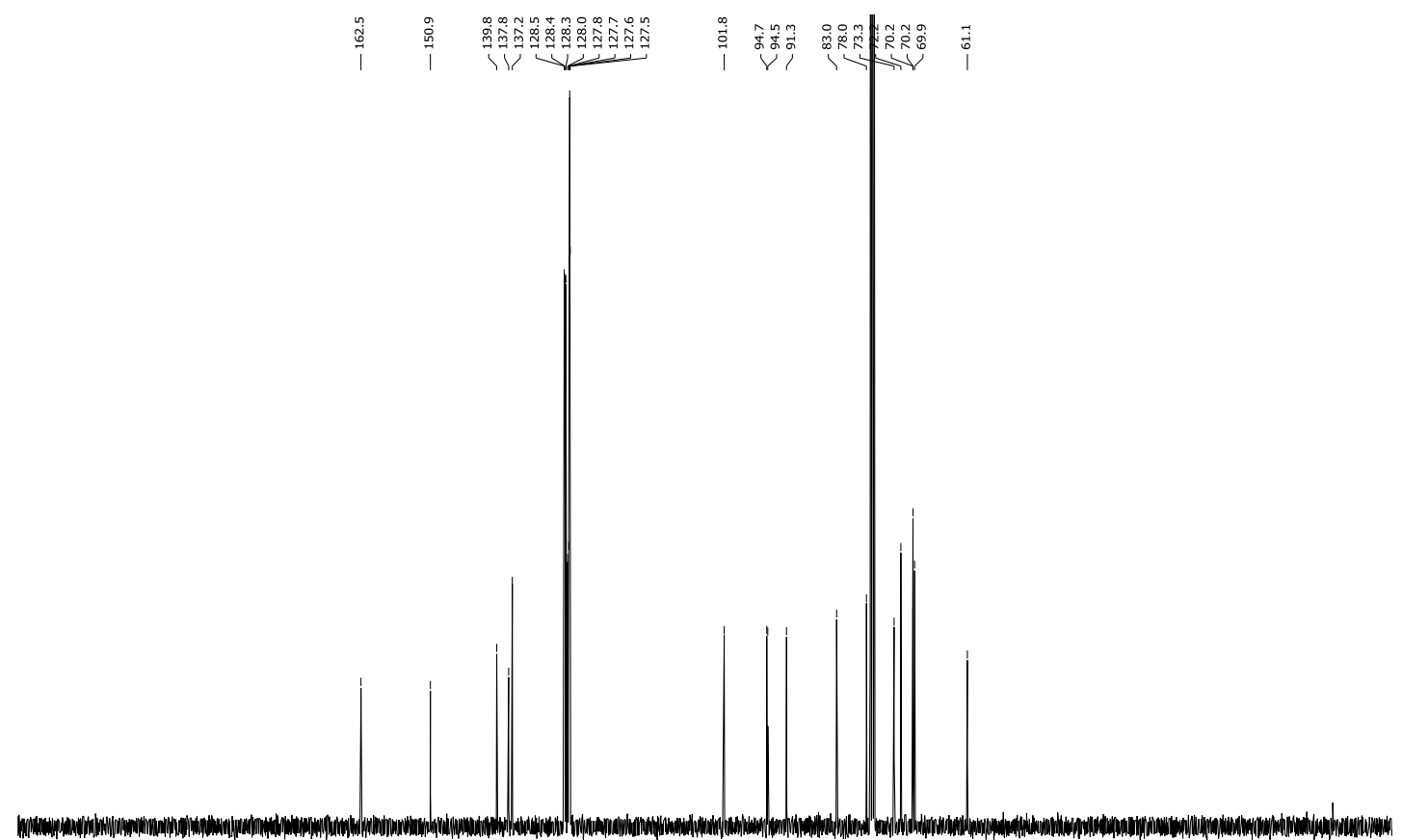

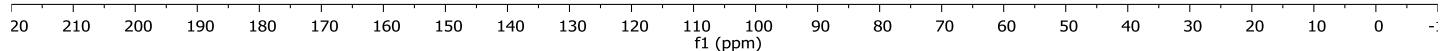

Figure S6. ${ }^{13} \mathrm{C}$ NMR $\left(101 \mathrm{MHz}, \mathrm{CDCl}_{3}\right)$ spectrum of compound 3. 


\section{Compound 4}

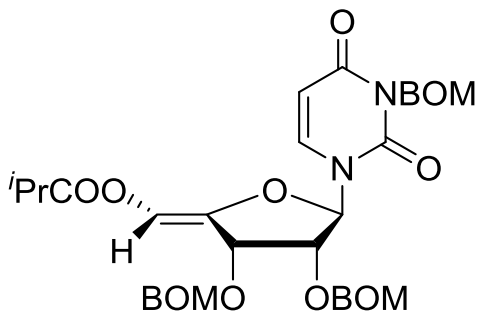

IBX (5.74 g, $20.5 \mathrm{mmol}, 2.50$ eq.) was added to a solution of alcohol $3(5.02 \mathrm{~g}, 8.30 \mathrm{mmol}$, 1.00 eq.) in acetonitrile $(100 \mathrm{~mL})$. The suspension was refluxed for $1 \mathrm{~h}$ and finally filtered through a plug of Celite $545 \AA$. The plug was rinsed with ethyl acetate $(3 \mathrm{x}, 50.0 \mathrm{~mL})$ and the solvent evaporated. The remaining aldehyde was received as a colorless foam and immediately dissolved with acetonitrile $(100 \mathrm{~mL})$. Potassium carbonate $(6.88 \mathrm{~g}, 49.8 \mathrm{mmol}, 6.00 \mathrm{eq}$.) and isobutyric anhydride (8.26 mL, $49.8 \mathrm{mmol}, 6.00$ eq.) were added and the reaction mixture refluxed for additional $3 \mathrm{~h}$. Finally, the reaction was terminated by the addition of ethyl acetate $(100 \mathrm{~mL})$ and water $(100 \mathrm{~mL})$. The aqueous layer was extracted with ethyl acetate $(3 \mathrm{x}, 100 \mathrm{~mL}$,) and the combined organic phases were dried over $\mathrm{Na}_{2} \mathrm{SO}_{4}$, filtered and evaporated to dryness. Purification by column chromatography $\left(\mathrm{SiO}_{2}\right.$, pentane/ethyl acetate, 10:1 to 1:1) afforded the exocyclic enol ester 4 as a single diastereomer (3.60 g, $5.35 \mathrm{mmol}, 54 \%$ yield).

${ }^{1} \mathbf{H}$ NMR $\left(500 \mathrm{MHz} \mathrm{CDCl}_{3}\right) \delta(\mathrm{ppm}) 7.38-7.26(\mathrm{~m}, 12 \mathrm{H}), 7.26-7.20(\mathrm{~m}, 3 \mathrm{H}), 7.06(\mathrm{~d}, J=8.1$ $\mathrm{Hz}, 1 \mathrm{H}), 6.82(\mathrm{~d}, J=0.6 \mathrm{~Hz}, 1 \mathrm{H}), 6.43(\mathrm{~d}, J=6.4 \mathrm{~Hz}, 1 \mathrm{H}), 5.75(\mathrm{~d}, J=8.1 \mathrm{~Hz}, 1 \mathrm{H}), 5.41(\mathrm{~d}, J=$ $9.7 \mathrm{~Hz}, 1 \mathrm{H}), 5.37(\mathrm{~d}, J=9.7 \mathrm{~Hz}, 1 \mathrm{H}), 4.96(\mathrm{~d}, J=7.1 \mathrm{~Hz}, 1 \mathrm{H}), 4.89-4.84(\mathrm{~m}, 2 \mathrm{H}), 4.80(\mathrm{~d}, J=$ $7.1 \mathrm{~Hz}, 1 \mathrm{H}), 4.74(\mathrm{~d}, J=4.8 \mathrm{~Hz}, 1 \mathrm{H}), 4.69-4.62(\mathrm{~m}, 4 \mathrm{H}), 4.60(\mathrm{~d}, J=12.0 \mathrm{~Hz}, 1 \mathrm{H}), 4.55(\mathrm{~d}, J=$ $12.0 \mathrm{~Hz}, 1 \mathrm{H}$ ), 4.49 (dd, $J=6.4,4.9 \mathrm{~Hz}, 1 \mathrm{H}$ ), 2.68 (hept, $J=7.0 \mathrm{~Hz}, 1 \mathrm{H}), 1.21$ (d, $J=7.0 \mathrm{~Hz}, 3 \mathrm{H}$ ), $1.21(\mathrm{~d}, J=7.0 \mathrm{~Hz}, 3 \mathrm{H})$.

${ }^{13} \mathrm{C}$ NMR (126 MHz, $\left.\mathrm{CDCl}_{3}\right) \delta(\mathrm{ppm})$ 173.5, 162.0, 150.9, 138.9, 137.9, 137.7, 137.2, 136.8, 128.5, 128.3, 128.0, 127.9, 127.7, 127.7, 127.6, 127.4, 116.4, 103.2, 94.4, 92.4, 89.5, 72.2, 71.2, 70.3, $70.2,70.1,33.7,18.9,18.8$.

IR (ATR): $\tilde{v}\left(\mathrm{~cm}^{-1}\right)=3092,3064,3032,2973,2941,2896,1747,1722,1674,1496,1452,1409$, 1353, 1265, 1237, 1176, 1134, 1092, 1067, 1021.

HR-MS (ESI): $m / z$ calcd for $\mathrm{C}_{37} \mathrm{H}_{40} \mathrm{~N}_{2} \mathrm{O}_{10} \mathrm{Na}^{+}:$: 695.25752; found: 695.25745 .

$[\alpha]_{\mathrm{D}}^{20}=-45.1^{\circ}\left(\mathrm{c}=0.26, \mathrm{CHCl}_{3}\right)$. 


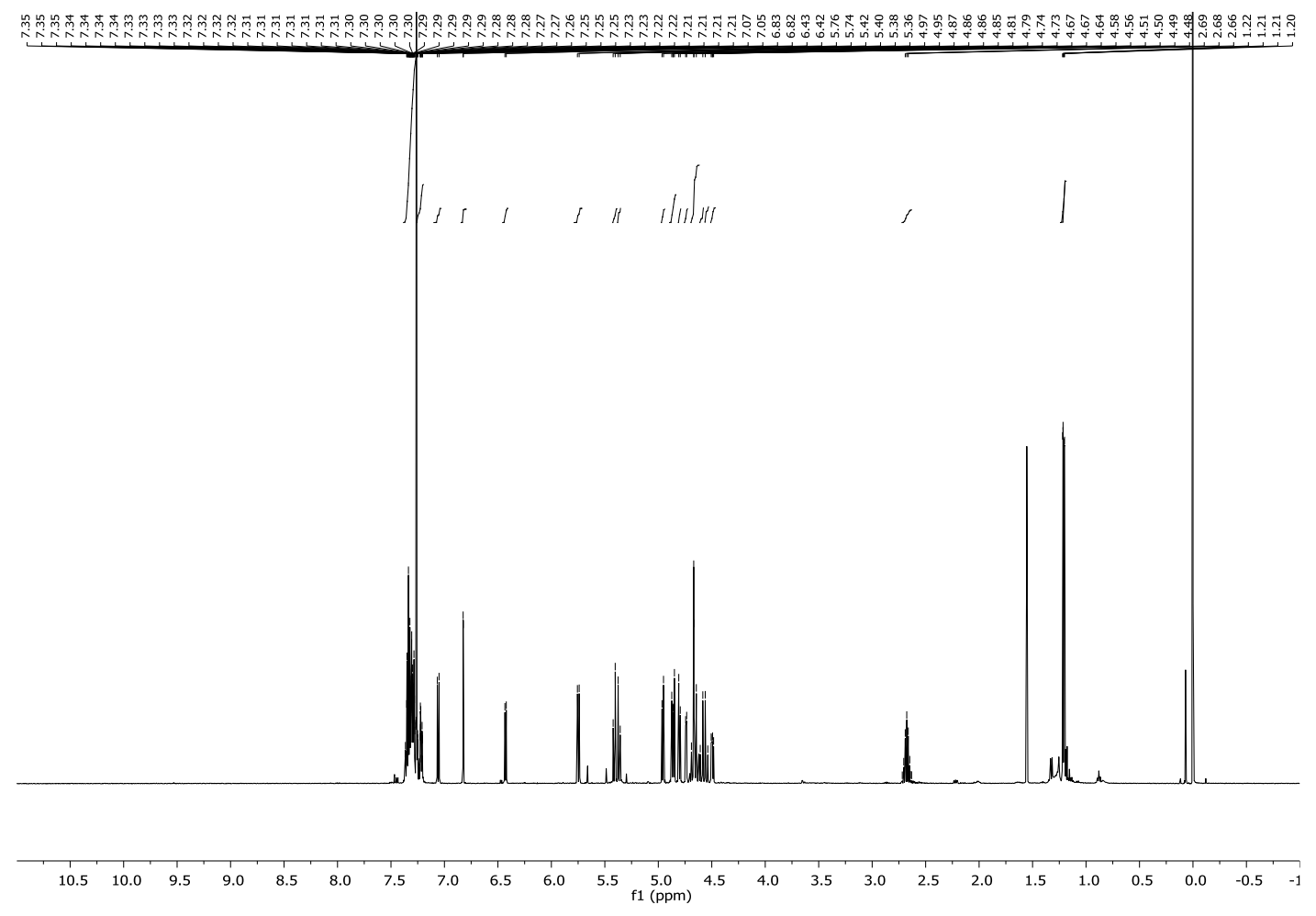

Figure S7. ${ }^{1} \mathrm{H}$ NMR $\left(500 \mathrm{MHz}, \mathrm{CDCl}_{3}\right)$ spectrum of compound 4.

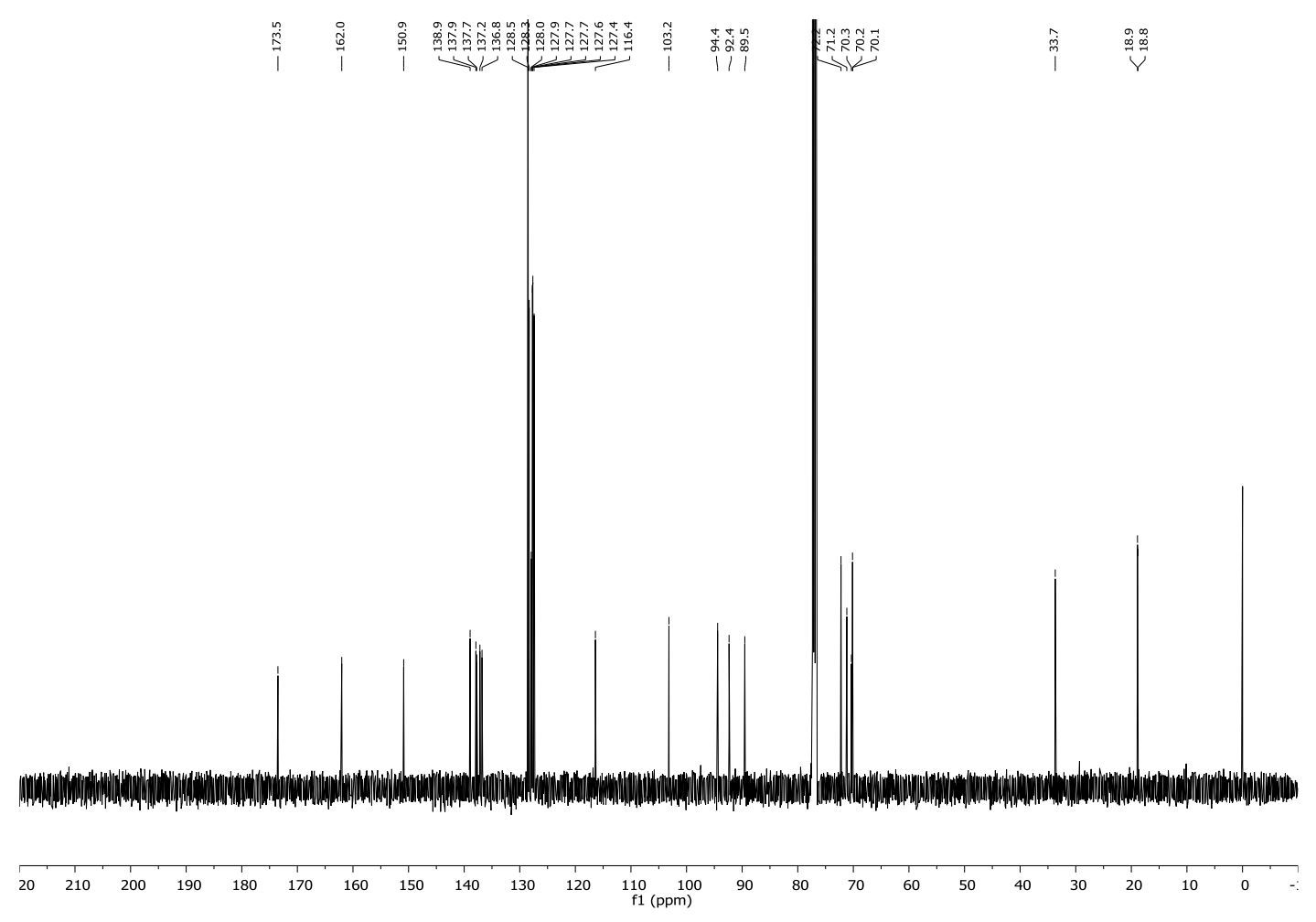

Figure S8. ${ }^{13} \mathrm{C}$ NMR (126 MHz, $\left.\mathrm{CDCl}_{3}\right)$ spectrum of compound 4. 


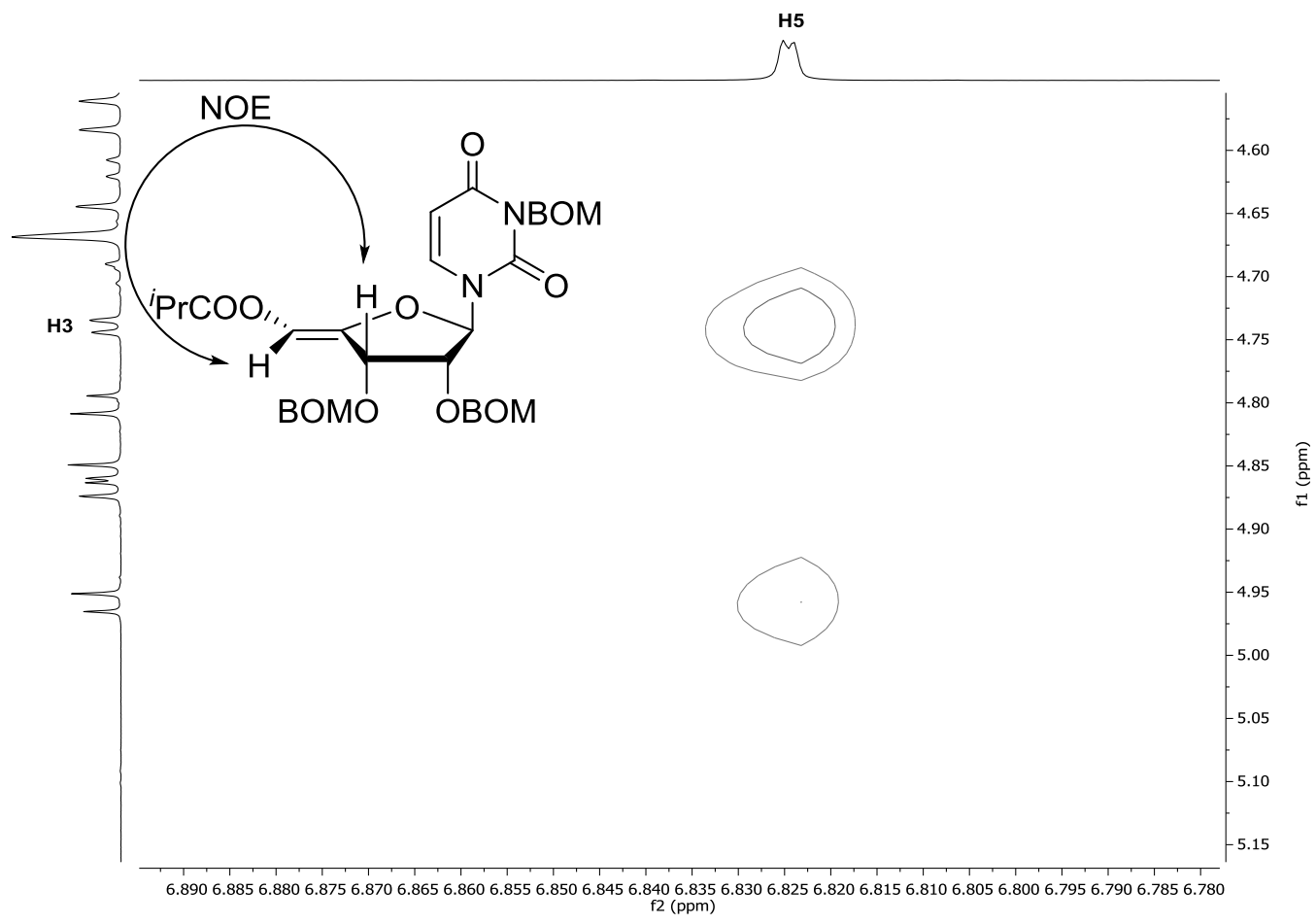

Figure S9. Partial NOESY (500 MHz, $\left.\mathrm{CDCl}_{3}\right)$ of compound 4. 


\section{Compound 5}

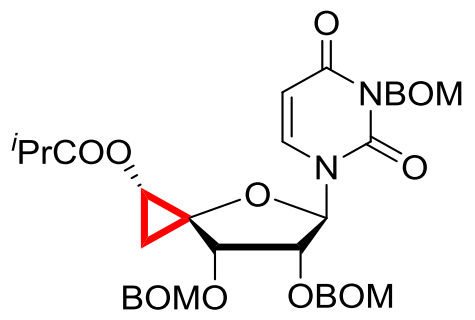

Before use, enol ester 4 was azeotropically distilled with toluene $(3 \mathrm{x}, 40.0 \mathrm{~mL})$ and dried for $0.5 \mathrm{~h}$ at high vacuum. To a solution of enol ester 4 (18.6 g, $27.6 \mathrm{mmol}, 1.00 \mathrm{eq}$.) in 1,2-dichloroethane (270 mL) was added molecular sieve (4 Å) and diiodomethane (22.3 mL, 277 mmol, 10.0 eq.). The suspension was stirred at room temperature for $0.5 \mathrm{~h}$ and finally a solution of diethylzinc ( $1 \mathrm{M}$ in hexane, $138 \mathrm{~mL}, 138 \mathrm{mmol}, 5.0$ eq.) was added dropwise. The reaction mixture was heated to $50{ }^{\circ} \mathrm{C}$ and stirred for $2 \mathrm{~h}$. Finally, reaction mixture was diluted with dichloromethane $(500 \mathrm{~mL})$, filtered and quenched with a saturated, aqueous solution of $\mathrm{Na}_{2} \mathrm{CO}_{3}(500 \mathrm{~mL})$. The aqueous layer was extracted with dichloromethane $(3 \mathrm{x}, 200 \mathrm{~mL})$, the combined organic phases dried over $\mathrm{Na}_{2} \mathrm{SO}_{4}$ and evaporated to dryness. Purification by column chromatography $\left(\mathrm{SiO}_{2}\right.$, pentane/ethyl acetate, $10: 1$ to $1: 1$ ) afforded the spirocyclopropanated uridine derivative 5 (10.3 g, $15.0 \mathrm{mmol}$, $54 \%$ yield) as a yellowish oil. For yet unknown reasons the afforded yields for different batches showed a range from $33 \%$ to $54 \%$.

${ }^{1} \mathrm{H}$ NMR $\left(500 \mathrm{MHz}, \mathrm{C}_{6} \mathrm{D}_{6}\right) \delta(\mathrm{ppm}) 7.38(\mathrm{~d}, J=8.2 \mathrm{~Hz}, 1 \mathrm{H}), 7.27(\mathrm{~m}, 2 \mathrm{H}), 7.22(\mathrm{~m}, 2 \mathrm{H}), 7.20-$ $7.17(\mathrm{~m}, 3 \mathrm{H}), 7.15-6.99(\mathrm{~m}, 8 \mathrm{H}), 6.86(\mathrm{~d}, J=7.2 \mathrm{~Hz}, 1 \mathrm{H}), 5.64(\mathrm{dd}, J=8.2,0.4 \mathrm{~Hz}, 1 \mathrm{H}), 5.38$ $5.32(\mathrm{~m}, 2 \mathrm{H}), 4.65(\mathrm{~d}, J=7.1 \mathrm{~Hz}, 1 \mathrm{H}), 4.62(\mathrm{~s}, 2 \mathrm{H}), 4.56(\mathrm{~d}, J=7.1 \mathrm{~Hz}, 1 \mathrm{H}), 4.54-4.41(\mathrm{~m}, 5 \mathrm{H})$, $4.36(\mathrm{~d}, J=12.1 \mathrm{~Hz}, 1 \mathrm{H}), 4.33(\mathrm{~d}, J=12.3 \mathrm{~Hz}, 1 \mathrm{H}), 3.78(\mathrm{~d}, J=4.5 \mathrm{~Hz}, 1 \mathrm{H}$ ), 3.50 (dd, $J=7.5,5.1$ $\mathrm{Hz}, 1 \mathrm{H}), 2.30$ (hept, $J=7.0 \mathrm{~Hz}, 1 \mathrm{H}), 1.20-1.11(\mathrm{~m}, 2 \mathrm{H}), 0.98$ (d, J = 7.0 Hz, 3H), 0.95 (d, J= 7.0 $\mathrm{Hz}, 3 \mathrm{H})$.

${ }^{13} \mathrm{C}$ NMR $\left(126 \mathrm{MHz}, \mathrm{C}_{6} \mathrm{D}_{6}\right) \delta 179.5,162.8,152.8,139.9,139.2,139.1,138.7,129.7,129.7,129.4$, 129.3, 128.7, 128.5, 103.7, 95.8, 95.3, 87.8, 80.0, 78.5, 73.2, 71.7, 70.8, 70.8, 67.9, 54.6, 34.8, $19.9,19.8,14.1,1.0$.

IR (ATR): $\tilde{v}\left(\mathrm{~cm}^{-1}\right)=3090,3064,3032,2972,2940,2896,1721,1672,1496,1455,1410,1354$, 1291, 1256, 1210, 1158, 1068, 1024.

HR-MS (ESI): $m / z$ calcd for $\mathrm{C}_{38} \mathrm{H}_{42} \mathrm{~N}_{2} \mathrm{O}_{10} \mathrm{Na}^{+}:$709.27317; found: 709.27332 .

$[\alpha]_{\mathrm{D}}^{20}=22.7^{\circ}\left(\mathrm{c}=0.66, \mathrm{CHCl}_{3}\right)$. 

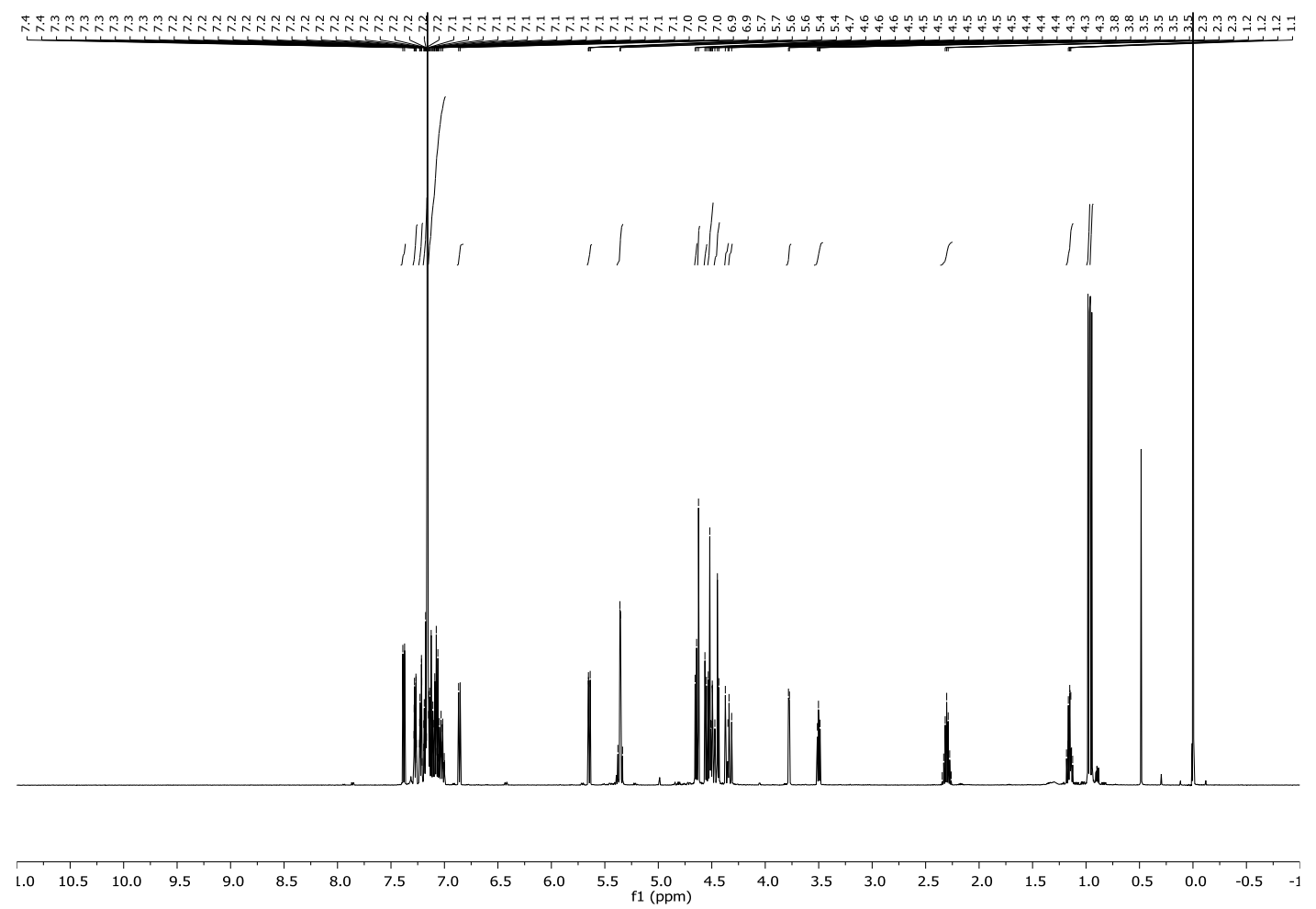

Figure S10. ${ }^{1} \mathrm{H}$ NMR $\left(500 \mathrm{MHz}, \mathrm{C}_{6} \mathrm{D}_{6}\right)$ spectrum of compound 5.

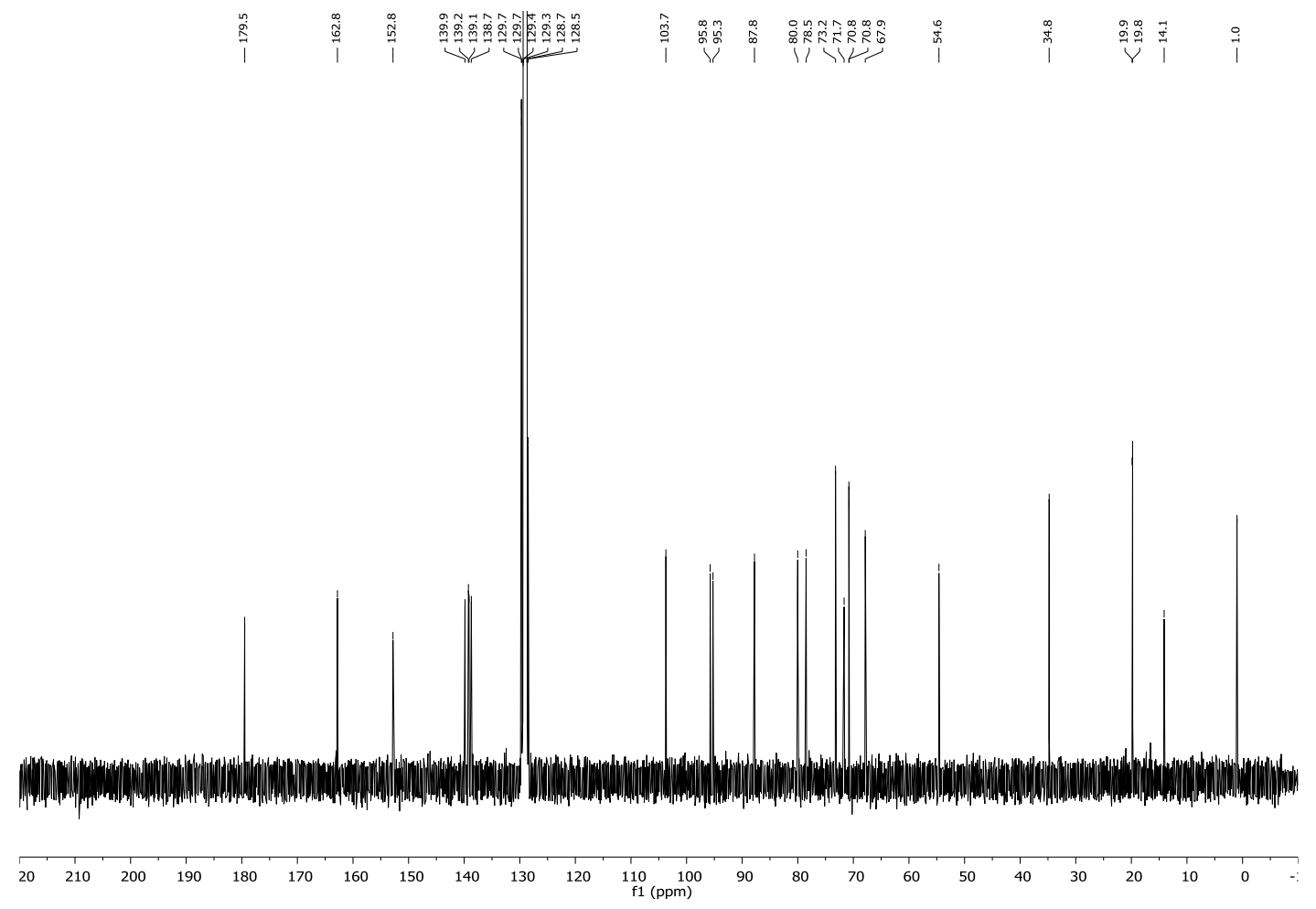

Figure S11. ${ }^{13} \mathrm{C}$ NMR (126 MHz, $\left.\mathrm{C}_{6} \mathrm{D}_{6}\right)$ spectrum of compound 5. 


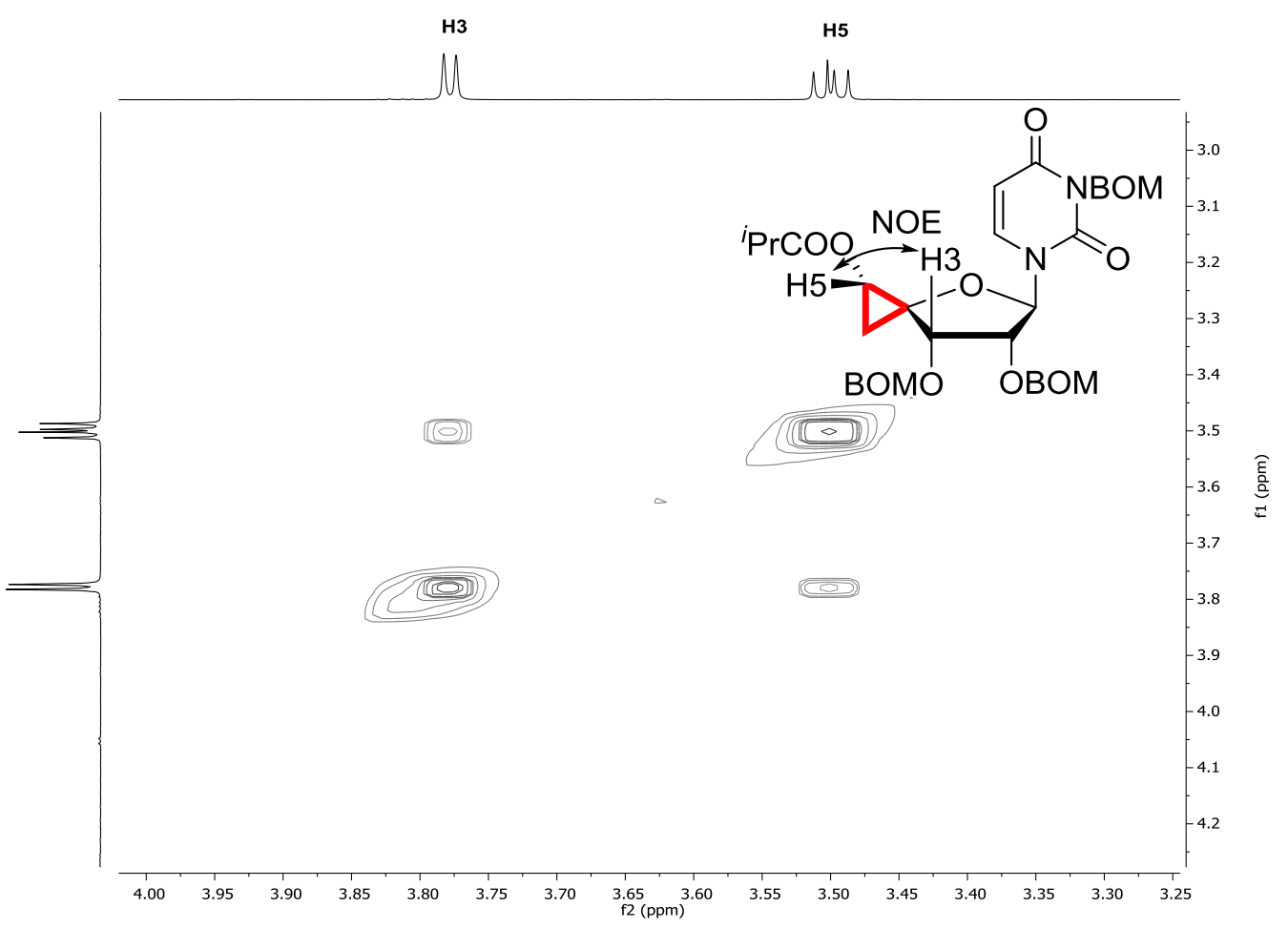

Figure S12. Partial NOESY $\left(500 \mathrm{MHz}, \mathrm{C}_{6} \mathrm{D}_{6}\right)$ of compound $\mathbf{5}$. 


\section{Compound 6}

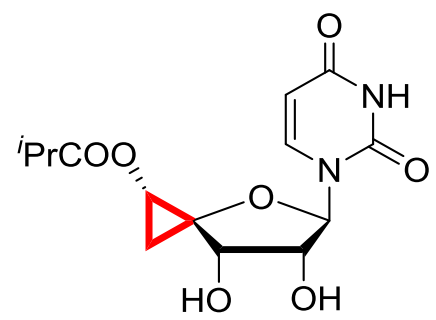

Under an argon atmosphere, derivative 5 (42.0 mg, $61.2 \mu \mathrm{mol}, 1.00$ eq.) was dissolved in methanol $(0.60 \mathrm{~mL})$. Palladium on charcoal (10 wt.\% on activated carbon, $13.0 \mathrm{mg}, 12.2 \mu \mathrm{mol}$, 0.2 eq.) was added and the argon atmosphere exchanged by a hydrogen atmosphere. The suspension was stirred at room temperature for $3.5 \mathrm{~h}$ and the reaction mixture finally filtered through Celite $\AA 545$. The Celite $\AA$ plug was rinsed with methanol $(3 x, 1.00 \mathrm{~mL})$ and evaporation of the solvent afforded ester 6 (19.9 mg, $60.9 \mu \mathrm{mol}$, quant) as a colorless foam.

For biological investigations compound 6 was previously purified by HPLC (C18, isocratic, $\left.\mathrm{CH}_{3} \mathrm{CN}: \mathrm{H}_{2} \mathrm{O}, 20: 80, t_{\mathrm{R}}=10.29 \mathrm{~min}\right)$.

${ }^{1} \mathrm{H}$ NMR (300 MHz, CD $\left.{ }_{3} \mathrm{OD}\right) \delta(\mathrm{ppm}) 7.55(\mathrm{~d}, J=8.1 \mathrm{~Hz}, 1 \mathrm{H}), 6.24(\mathrm{~d}, J=7.7 \mathrm{~Hz}, 1 \mathrm{H}), 5.73$ (dd, $J=8.1,0.6 \mathrm{~Hz}, 1 \mathrm{H}), 4.48$ (dd, $J=7.7,4.8 \mathrm{~Hz}, 1 \mathrm{H}), 3.91$ (d, $J=4.8 \mathrm{~Hz}, 1 \mathrm{H}), 3.89$ (dd, $J=7.7,4.5$ $\mathrm{Hz}, 1 \mathrm{H}), 2.59$ (hept, $J=7.0 \mathrm{~Hz}, 1 \mathrm{H}), 1.40$ (dd, $J=8.6,7.8 \mathrm{~Hz}, 1 \mathrm{H}), 1.16(\mathrm{~d}, J=7.0 \mathrm{~Hz}, 3 \mathrm{H}), 1.12$ (d, $J=7.0 \mathrm{~Hz}, 3 \mathrm{H}), 1.09$ (dd, $J=8.8,4.7 \mathrm{~Hz}, 1 \mathrm{H}$ ).

${ }^{13} \mathrm{C}$ NMR (151 MHz, CD $\left.{ }_{3} \mathrm{OD}\right) \delta(\mathrm{ppm}) 180.0,165.8,152.8,142.1,103.4,88.2,75.0,74.4,69.4$, 54.7, 34.8, 19.3, 19.2, 12.8 .

IR (ATR): $\tilde{v}\left(\mathrm{~cm}^{-1}\right)=3354,3182,3103,3071,2983,2939,2913,2882,2836,1744,1688,1659$, 1473, 1395, 1252, 1187, 1045.

HR-MS (ESI): $m / z$ calcd for $\mathrm{C}_{14} \mathrm{H}_{18} \mathrm{~N}_{2} \mathrm{O}_{7} \mathrm{Na}^{+}: 349.10062$; found: 349.10067 .

$[\alpha]_{\mathrm{D}}^{20}=-24.1^{\circ}(\mathrm{c}=0.71, \mathrm{MeOH})$. 


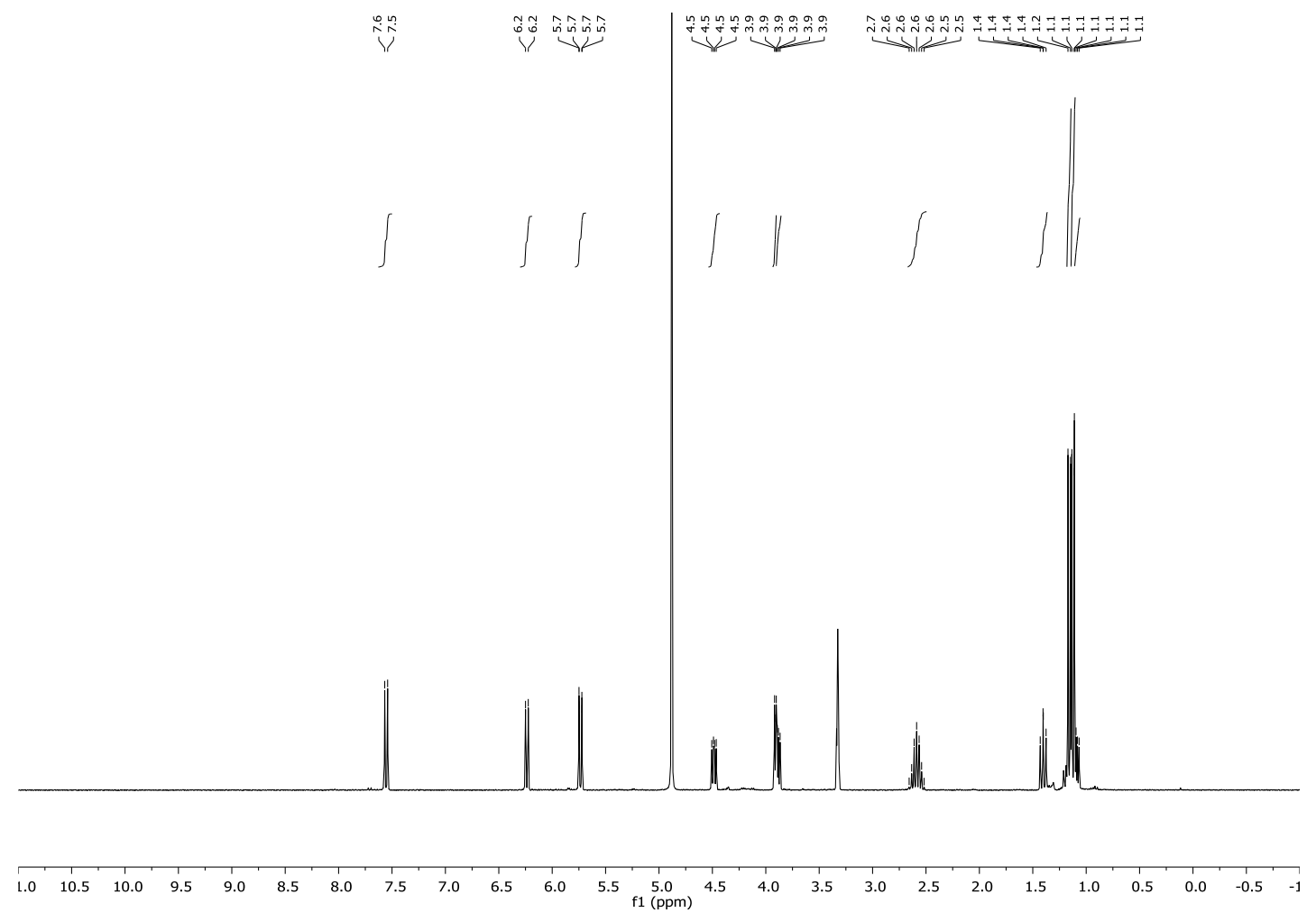

Figure S13. ${ }^{1} \mathrm{H} \mathrm{NMR}\left(300 \mathrm{MHz}, \mathrm{CD}_{3} \mathrm{OD}\right)$ spectrum of compound 6.

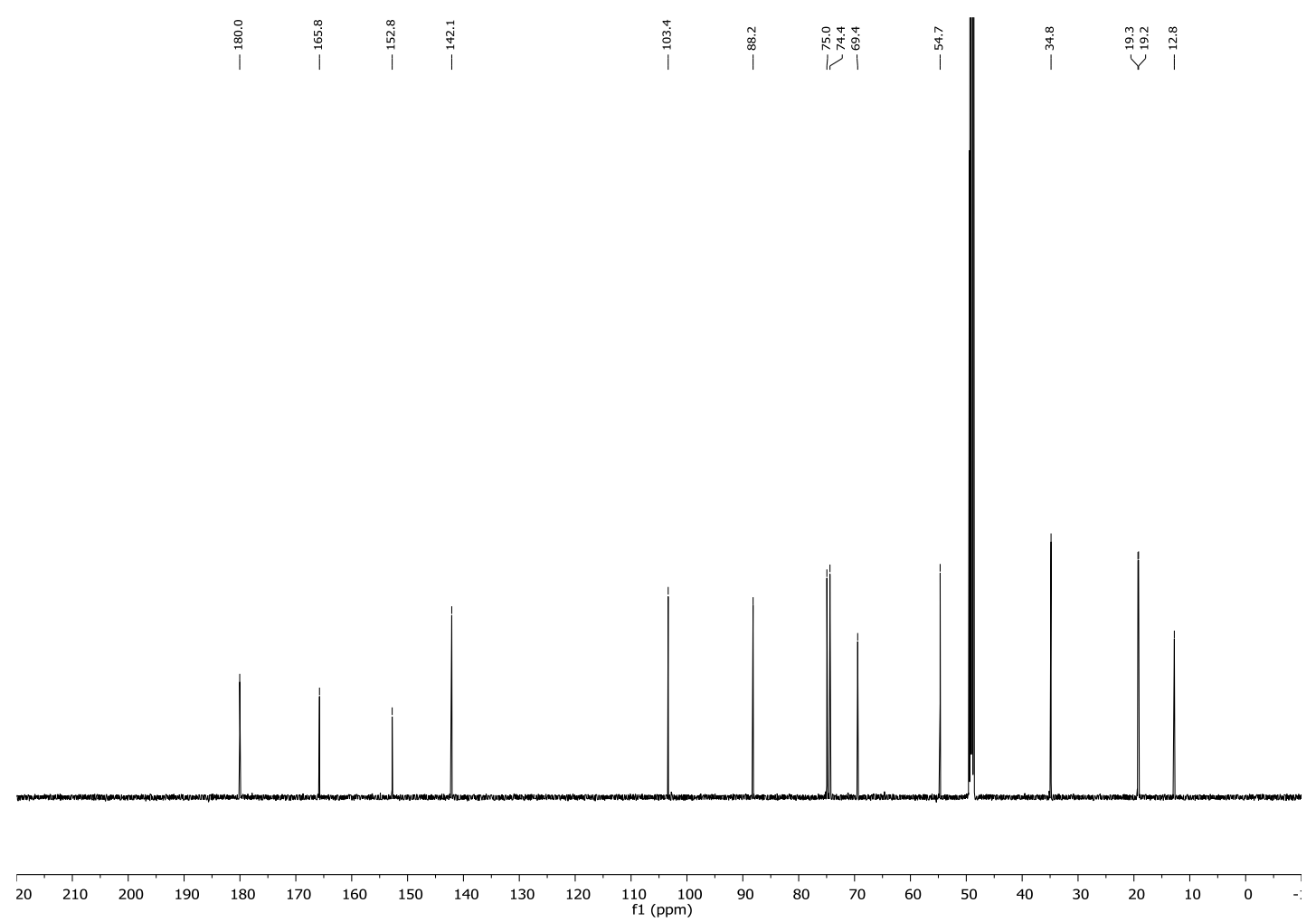

Figure S14. ${ }^{13} \mathrm{C}$ NMR (151 MHz, $\left.\mathrm{CD}_{3} \mathrm{OD}\right)$ spectrum of compound 6. 


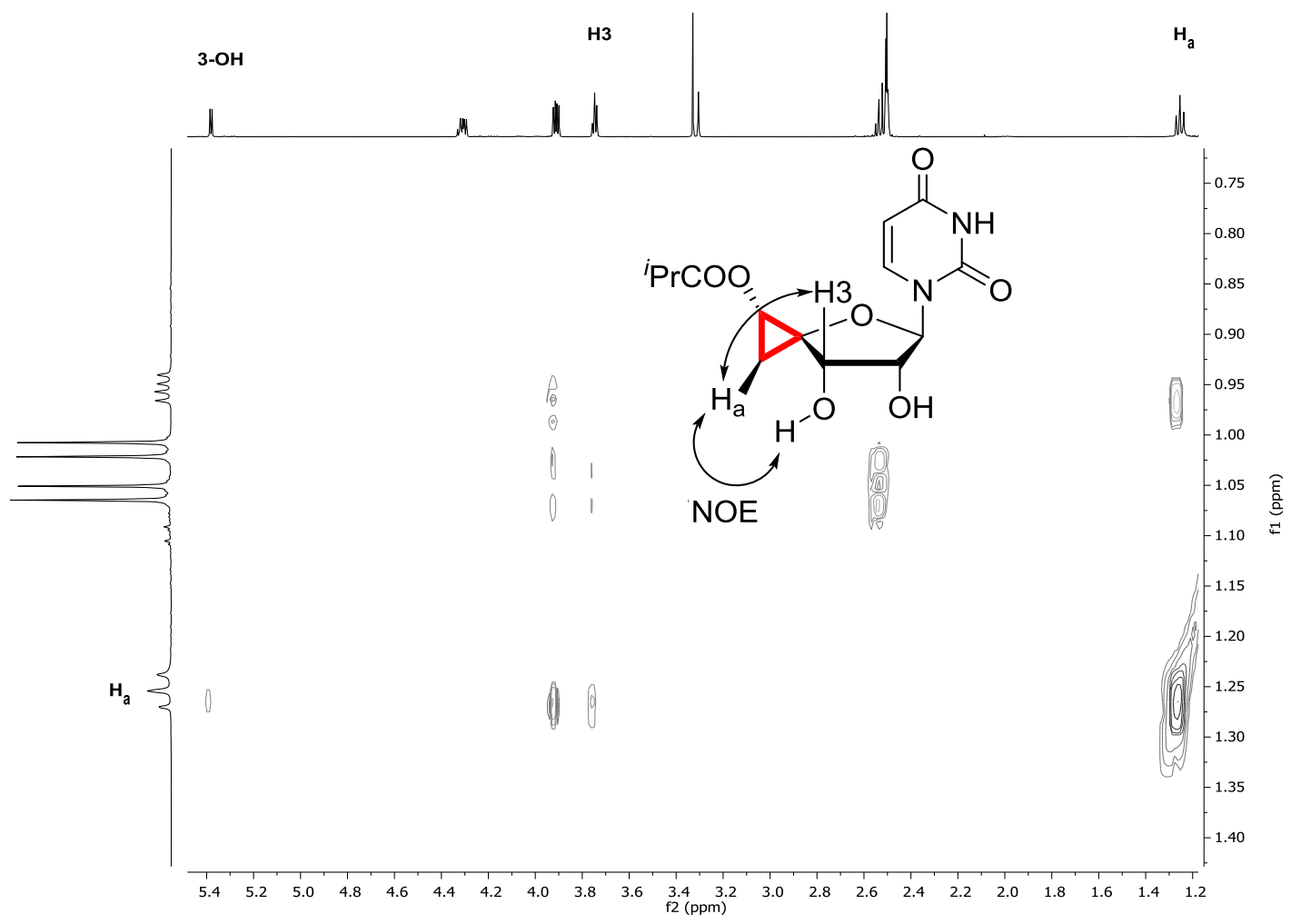

Figure S15. Partial NOESY (500 MHz, DMSO-d6) of compound 6.

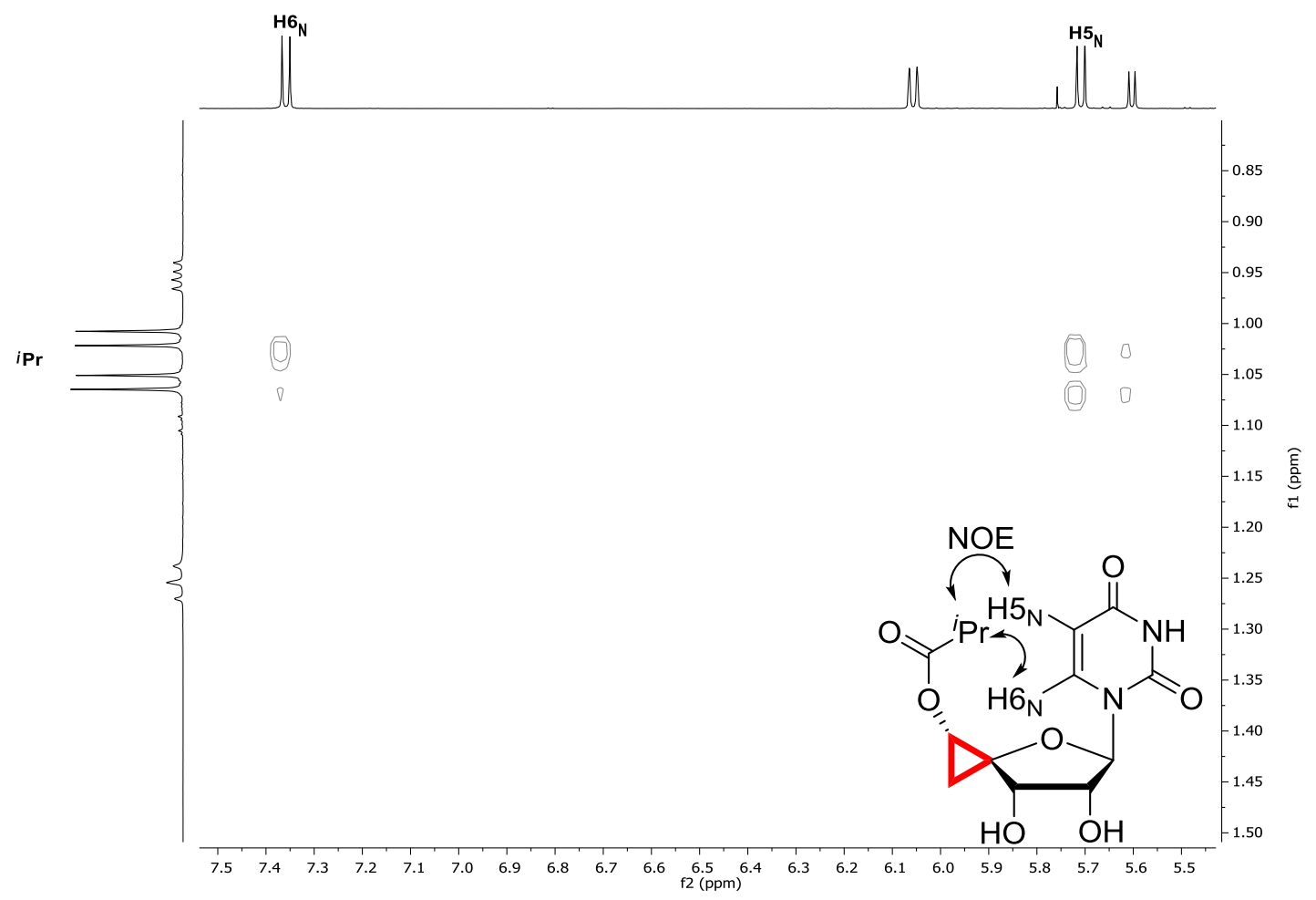

Figure 16. Partial NOESY $\left(300 \mathrm{MHz}, \mathrm{CD}_{3} \mathrm{OD}\right)$ of compound 6 . 


\section{Compound 7}

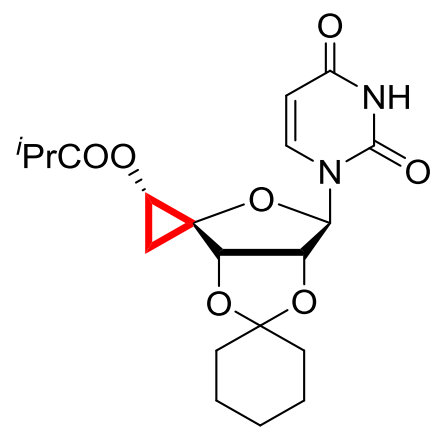

To a solution of derivative 6 (1.30 g, $3.98 \mathrm{mmol}, 1.00$ eq.) in 1,2-dichloroethane $(78.0 \mathrm{~mL})$ was added cyclohexanone dimethyl ketal $(2.12 \mathrm{~mL} 13.9 \mathrm{mmol}, 3.50 \mathrm{eq}$.) and p-toluenesulfonic acid (68.5 mg, $0.39 \mu \mathrm{mol}, 0.10 \mathrm{eq}$.). The reaction mixture was heated to $50^{\circ} \mathrm{C}$ and stirred for $3 \mathrm{~h}$. The reaction was terminated by the addition of $\mathrm{NEt}_{3}(0.30 \mathrm{~mL})$ and the solvent was evaporated to dryness. Purification of the remaining residue by column chromatography $\left(\mathrm{SiO}_{2}\right.$, pentane/ethyl acetate, 5:1 to 1:1) afforded the cyclohexylidene protected uridine derivative 7 (1.23 g, $3.03 \mathrm{mmol}$, $76 \%)$ as a colorless solid.

${ }^{1} \mathrm{H}$ NMR $\left(500 \mathrm{MHz}, \mathrm{CD}_{3} \mathrm{CN}\right) \delta(\mathrm{ppm}) 9.03(\mathrm{~s}, 1 \mathrm{H}), 7.32(\mathrm{~d}, J=8.1 \mathrm{~Hz}, 1 \mathrm{H}), 5.76(\mathrm{~d}, J=0.6 \mathrm{~Hz}$, $1 \mathrm{H}), 5.57(\mathrm{dd}, J=8.1,2.2 \mathrm{~Hz}, 1 \mathrm{H}), 5.25(\mathrm{dd}, J=5.9,0.7 \mathrm{~Hz}, 1 \mathrm{H}), 4.71(\mathrm{~d}, J=5.8 \mathrm{~Hz}, 1 \mathrm{H}), 4.03$ (dd, $J=7.6,4.6 \mathrm{~Hz}, 1 \mathrm{H}$ ), 2.40 (hept, $J=7.0 \mathrm{~Hz}, 1 \mathrm{H}), 1.77-1.70(\mathrm{~m}, 2 \mathrm{H}), 1.66-1.49(\mathrm{~m}, 6 \mathrm{H})$, $1.43-1.35$ (m, 3H), 1.07 (ddd, $J=8.5,4.6,0.5 \mathrm{~Hz}, 1 \mathrm{H}), 1.03$ (d, J=7.0 Hz, 3H), 1.01 (d, $J=7.0$ $\mathrm{Hz}, 3 \mathrm{H})$.

${ }^{13} \mathrm{C}$ NMR $\left(126 \mathrm{MHz}, \mathrm{CD}_{3} \mathrm{CN}\right) \delta$ (ppm) 177.6, 163.9, 151.5, 144.5, 114.4, 102.8, 94.9, 85.0, 84.4, $72.2,55.4,36.8,35.7,34.6,25.7,24.8,24.7,19.1,19.0,14.1$.

IR (ATR): $\tilde{v}\left(\mathrm{~cm}^{-1}\right)=3206,3092,3066,2935,2860,1685,1451,1372,1334,1257,1214,1157$, 1100, 1048.

HR-MS (ESI): $m / z$ calcd for $\mathrm{C}_{20} \mathrm{H}_{26} \mathrm{~N}_{2} \mathrm{O}_{7} \mathrm{Na}^{+}:$429.16322; found: 429.16350 .

$[\alpha]_{\mathrm{D}}^{20}=118.2^{\circ}\left(\mathrm{C}=0.44, \mathrm{CHCl}_{3}\right)$. 


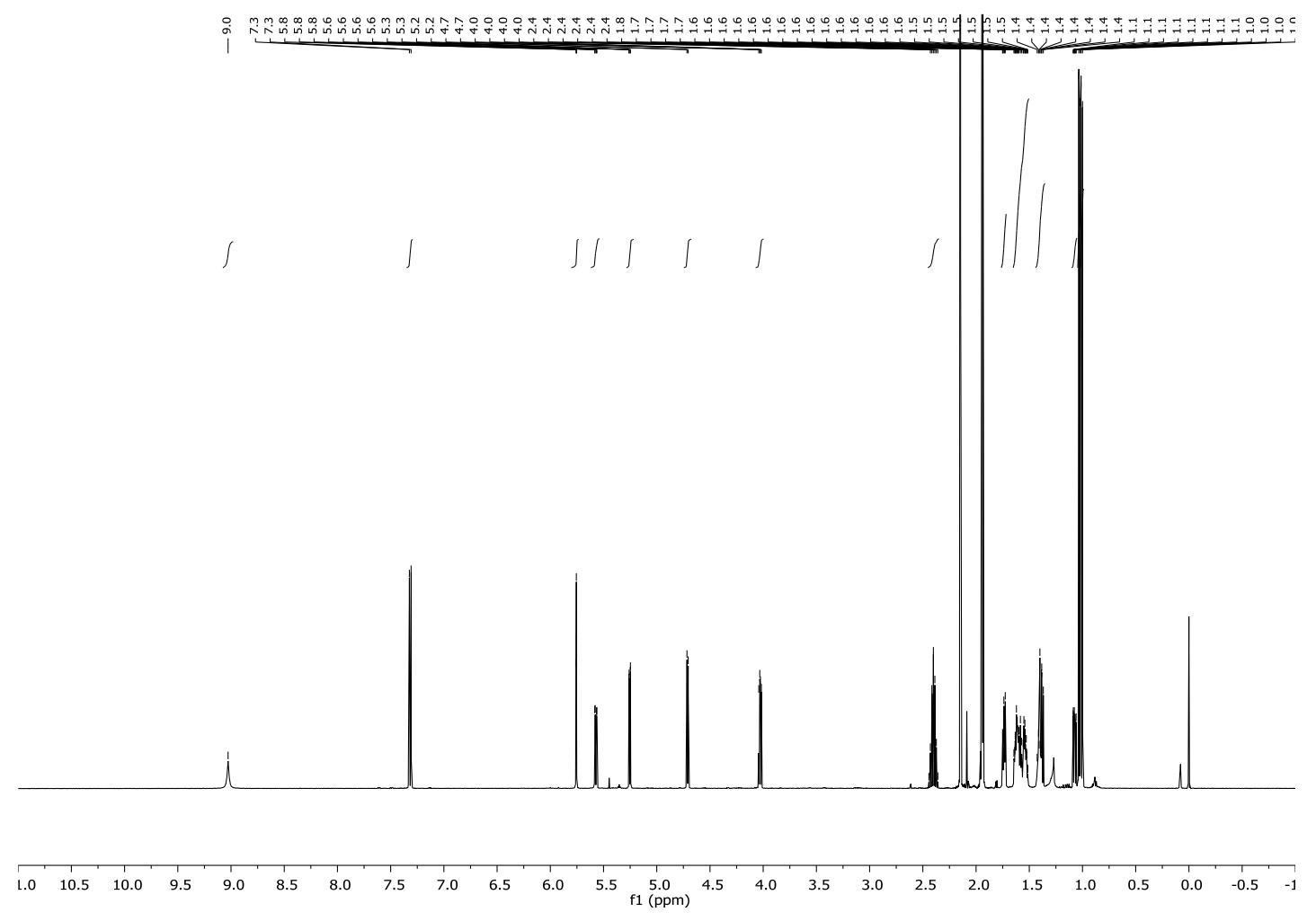

Figure S17. ${ }^{1} \mathrm{H}$ NMR $\left(500 \mathrm{MHz}, \mathrm{CD}_{3} \mathrm{CN}\right)$ spectrum of compound 7.

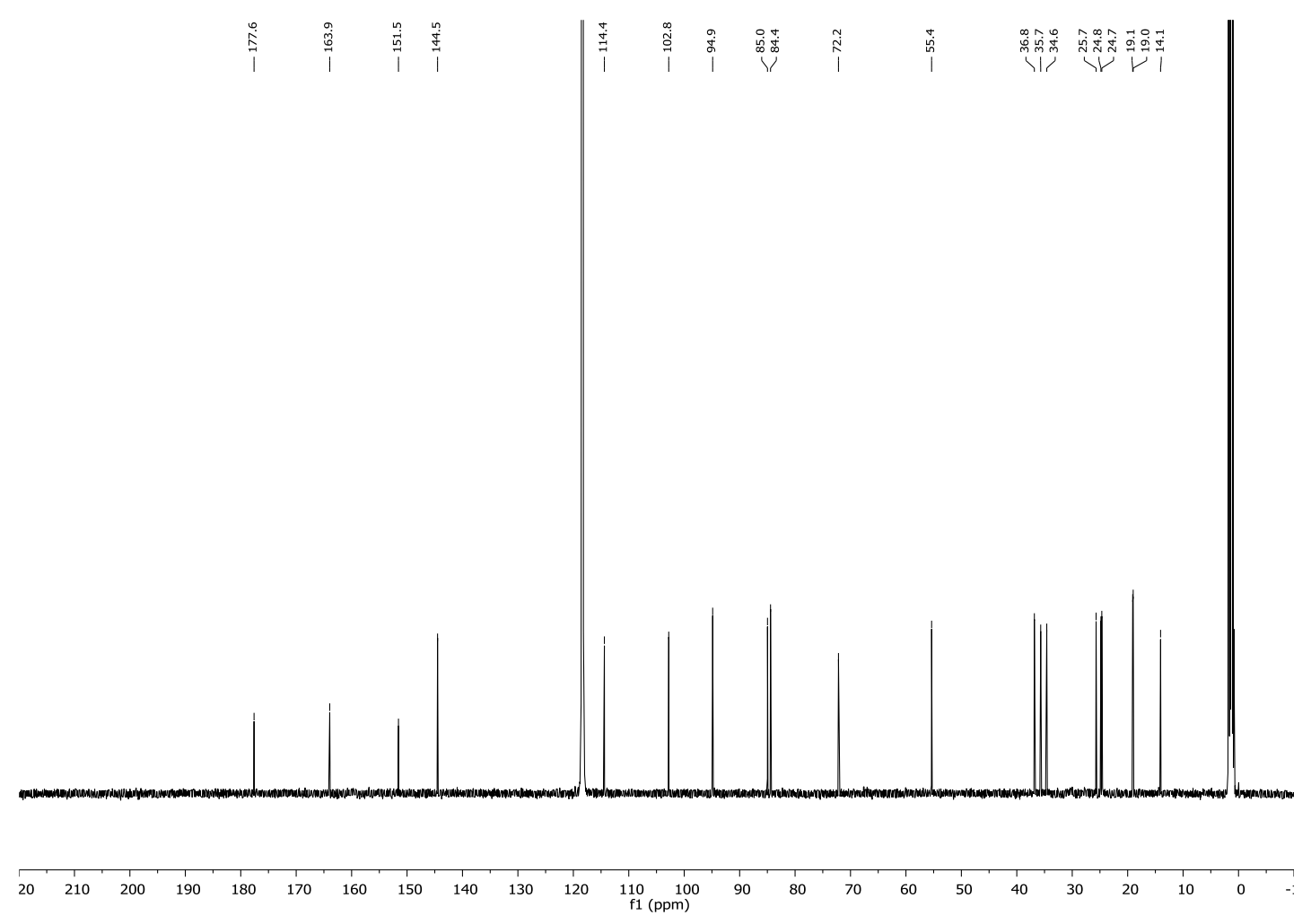

Figure S18. ${ }^{13} \mathrm{C}$ NMR (126 MHz, $\mathrm{CD}_{3} \mathrm{CN}$ ) spectrum of compound 7. 


\section{Compound 8}

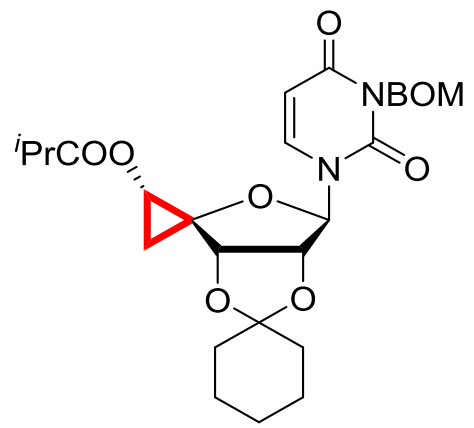

To a solution of compound 7 (1.23 $\mathrm{mg}, 3.03 \mathrm{mmol}, 1.00$ eq.) in dichloromethane $(40.0 \mathrm{~mL})$ was added NEt'Pr $\mathrm{Pr}_{2}\left(2.57 \mathrm{~mL}, 15.2 \mathrm{~mol}, 5.00\right.$ eq.) at $0{ }^{\circ} \mathrm{C}$. The mixture was stirred for $5 \mathrm{~min}$ and finally $\mathrm{BOMCl}(1.04,7.53 \mathrm{mmol}, 2.5 \mathrm{eq}$.) was added. The reaction was thawed overnight and quenched in a cooling bath with dichloromethane $(40.0 \mathrm{~mL})$ and water $(50.0 \mathrm{~mL})$. The aqueous layer was extracted with dichloromethane $(3 \mathrm{x}, 40.0 \mathrm{~mL})$. The combined organic layers were washed with brine $(100 \mathrm{~mL})$, dried over $\mathrm{Na}_{2} \mathrm{SO}_{4}$, filtered and evaporated to dryness. Purification by column chromatography (neutralized $\mathrm{SiO}_{2}$, pentane/ethyl acetate, 7:1 to 2:1) afforded the protected uridine derivative 8 (1.42 g, $2.70 \mathrm{mmol}, 89 \%$ yield) as a yellowish oil.

${ }^{1} \mathbf{H}$ NMR $\left(500 \mathrm{MHz}, \mathrm{CDCl}_{3}\right) \delta(\mathrm{ppm}) 7.40-7.27(\mathrm{~m}, 5 \mathrm{H}), 7.18(\mathrm{~d}, J=8.1 \mathrm{~Hz}, 1 \mathrm{H}), 5.75-5.71(\mathrm{~m}$, 2H), $5.46(\mathrm{~d}, J=9.7 \mathrm{~Hz}, 1 \mathrm{H}), 5.40(\mathrm{~d}, J=9.7 \mathrm{~Hz}, 1 \mathrm{H}), 5.18(\mathrm{dd}, J=6.0,1.2 \mathrm{~Hz}, 1 \mathrm{H}), 4.73(\mathrm{~d}, J=$ $6.0 \mathrm{~Hz}, 1 \mathrm{H}$ ), 4.68 (s, 2H), 4.02 (dd, $J=7.7,4.7 \mathrm{~Hz}, 1 \mathrm{H}$ ), 2.42 (hept, $J=7.0 \mathrm{~Hz}, 1 \mathrm{H}$ ), $1.78(\mathrm{~m}, 2 \mathrm{H}$ ), $1.68-1.54(\mathrm{~m}, 6 \mathrm{H}), 1.51(\mathrm{dd}, J=8.8,7.7 \mathrm{~Hz}, 1 \mathrm{H}), 1.41(\mathrm{~m}, 2 \mathrm{H}), 1.16(\mathrm{dd}, J=9.0,4.7 \mathrm{~Hz}, 1 \mathrm{H})$, $1.05(\mathrm{~d}, J=7.0 \mathrm{~Hz}, 3 \mathrm{H}), 1.01(\mathrm{~d}, J=7.0 \mathrm{~Hz}, 3 \mathrm{H})$.

${ }^{13} \mathrm{C}$ NMR (126 MHz, $\left.\mathrm{CDCl}_{3}\right) \delta(\mathrm{ppm})$ 177.1, 162.5, 150.9, 141.2, 137.8, 128.3, 127.7, 127.6, 114.4, 102.3, 95.1, 84.4, 83.0, 72.3, 71.8, 70.3, 54.5, 36.1, 35.1, 33.7, 24.9, 23.9, 23.7, 18.7, 18.7, 13.8. IR $(A T R): \tilde{v}\left(\mathrm{~cm}^{-1}\right)=2935,2860,1722,1669,1635,1540,1450,1353,1307,1257,1191,1157$, 1096.

HR-MS (ESI): $m / z$ calcd for $\mathrm{C}_{28} \mathrm{H}_{34} \mathrm{~N}_{2} \mathrm{O}_{8} \mathrm{Na}^{+}:$549.22074; found: 549.22084.

$[\alpha]_{\mathrm{D}}^{20}=110.7^{\circ}\left(\mathrm{c}=0.81, \mathrm{CHCl}_{3}\right)$. 

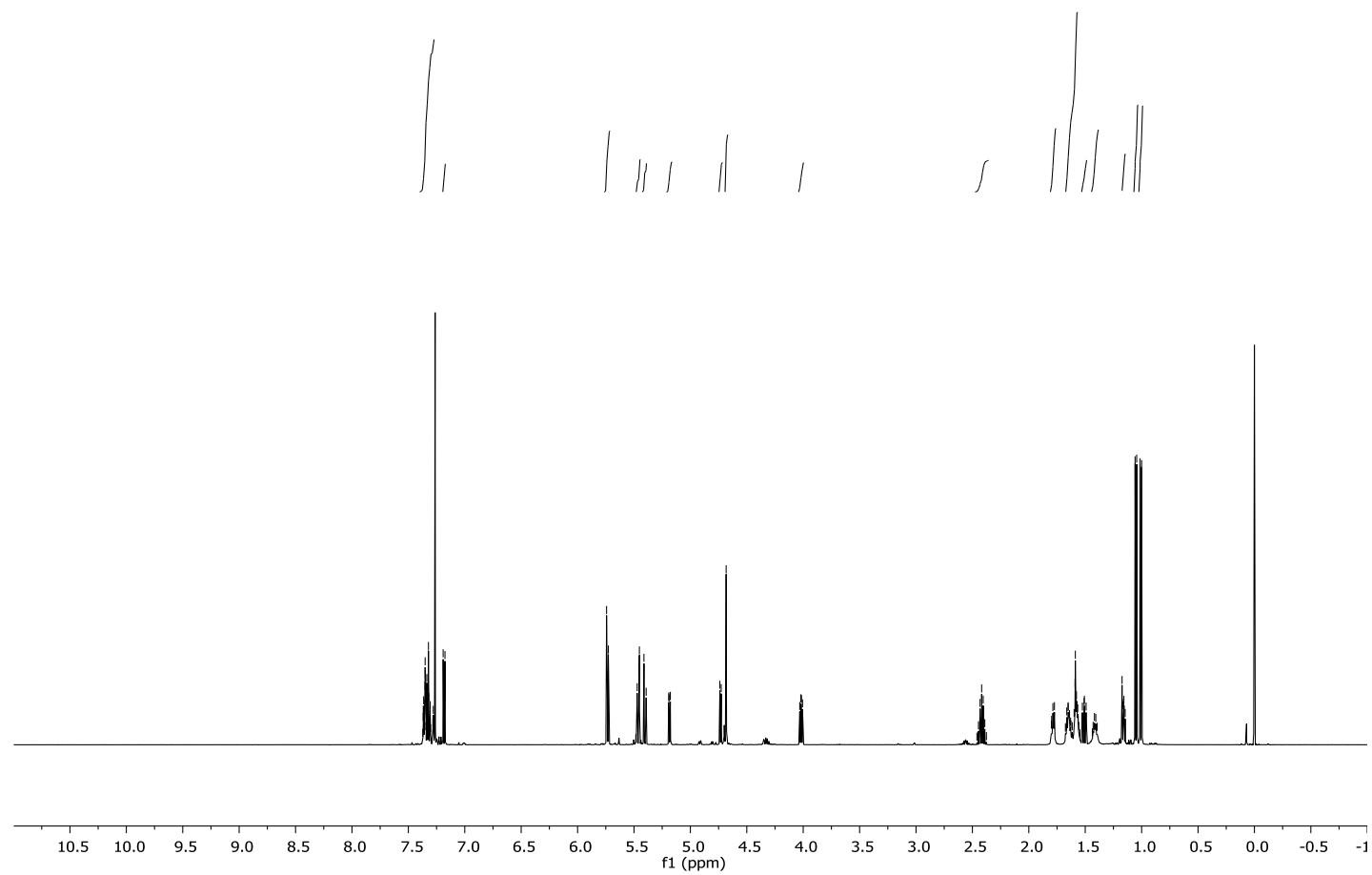

Figure S19. ${ }^{1} \mathrm{H}$ NMR $\left(500 \mathrm{MHz}, \mathrm{CDCl}_{3}\right)$ spectrum of compound 8.

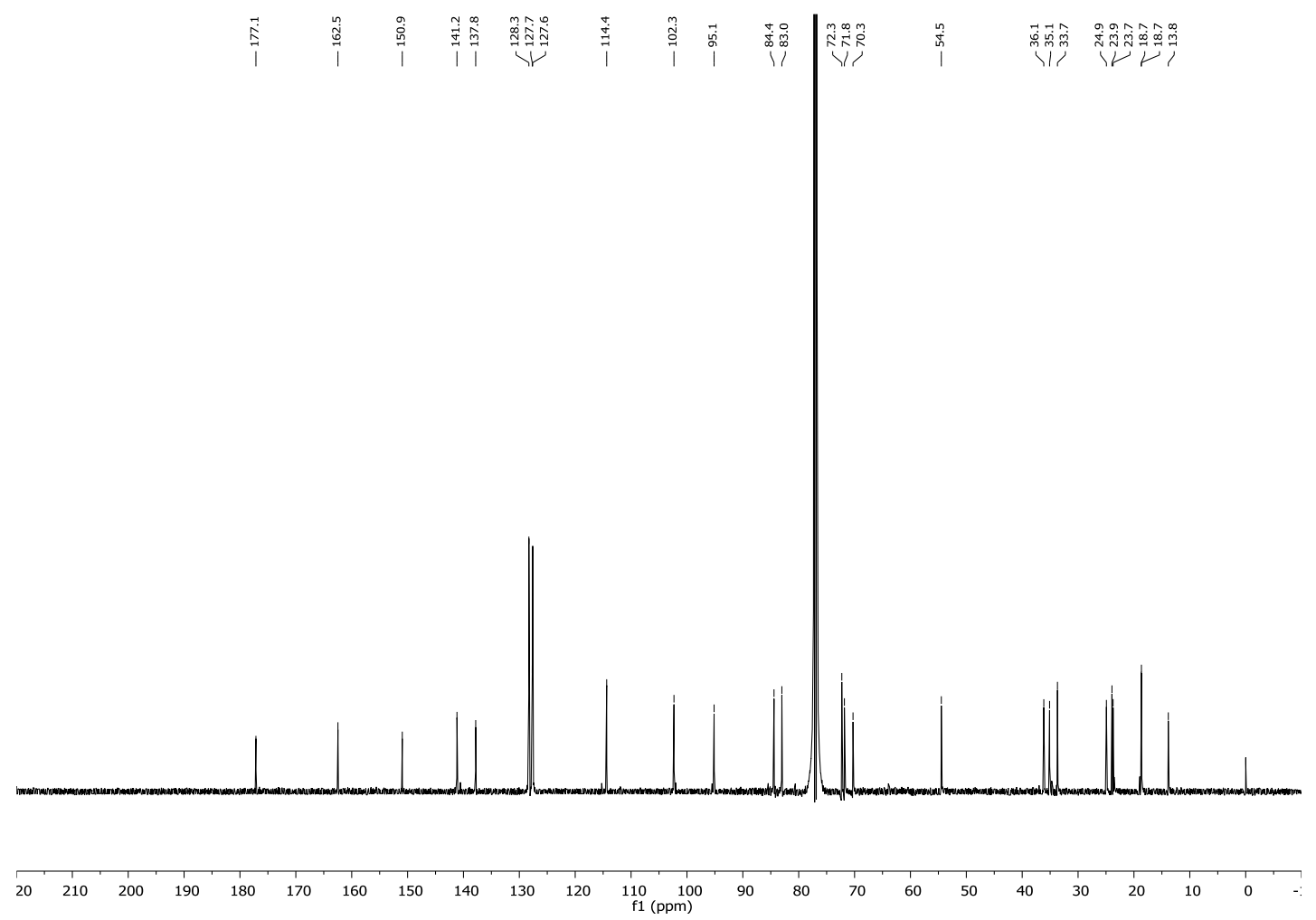

Figure S17. ${ }^{13} \mathrm{C}$ NMR (126 MHz, $\mathrm{CDCl}_{3}$ ) spectrum of compound 8. 


\section{Compound 9}

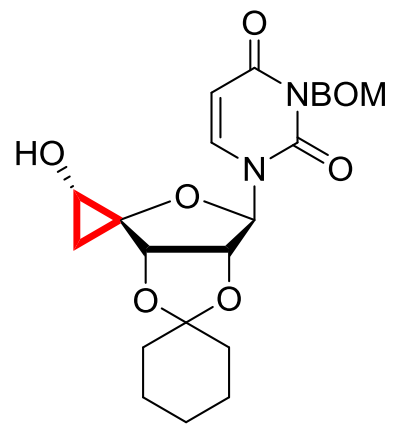

To a solution of compound 8 (1.42 g, $2.70 \mathrm{mmol}, 1.00 \mathrm{eq}$. ) in methanol (120 mL) was added $\mathrm{NaOMe}\left(5.40 \mathrm{M}\right.$ in methanol, $0.15 \mathrm{~mL}, 0.81 \mathrm{mmol}, 0.30 \mathrm{eq}$ ) at $0^{\circ} \mathrm{C}$. The reaction mixture was stirred for $10 \mathrm{~h}$ and after full consumption of starting material Amberlite® IR120 (hydrogen form) was added until neutralization was achieved. The polymer was removed by filtration, washed with methanol $(3 \mathrm{x}, 10.0 \mathrm{~mL})$ and the solvent removed at ambient temperature under reduced pressure. Cyclopropanol 9 was afforded as a colorless foam $(1.23 \mathrm{~g}, 2.69 \mathrm{mmol}$, quant.) and used for the next step without further purification. Column purification $\left(\mathrm{SiO}_{2}\right.$, pentane/ethyl acetate, $\left.1: 1\right)$ led to diminished yield of the desired spirocyclopropanol.

${ }^{1} \mathrm{H}$ NMR $\left(400 \mathrm{MHz}, \mathrm{CDCl}_{3}\right) \delta(\mathrm{ppm}) 7.40-7.26(\mathrm{~m}, 5 \mathrm{H}), 7.23(\mathrm{~d}, J=8.1 \mathrm{~Hz}, 1 \mathrm{H}), 5.75(\mathrm{~d}, J=8.0$ $\mathrm{Hz}, 1 \mathrm{H}), 5.49(\mathrm{~d}, J=9.7 \mathrm{~Hz}, 1 \mathrm{H}), 5.46-5.37(\mathrm{~m}, 2 \mathrm{H}), 5.24(\mathrm{dd}, J=6.0,1.3 \mathrm{~Hz}, 1 \mathrm{H}), 4.72-4.62$ $(\mathrm{m}, 2 \mathrm{H}), 4.58(\mathrm{~d}, J=6.1 \mathrm{~Hz}, 1 \mathrm{H}), 3.42(\mathrm{dd}, J=7.5,4.8 \mathrm{~Hz}, 1 \mathrm{H}), 2.42(\mathrm{~s}, 1 \mathrm{H}), 1.82-1.76(\mathrm{~m}, 2 \mathrm{H})$, $1.68-1.56(\mathrm{~m}, 6 \mathrm{H}), 1.42(\mathrm{~d}, J=6.9 \mathrm{~Hz}, 2 \mathrm{H}), 1.30$ (dd, J = 8.6, $7.4 \mathrm{~Hz}, 1 \mathrm{H}), 1.05$ (dd, J = 8.6, 4.8 $\mathrm{Hz}, 1 \mathrm{H})$.

${ }^{13} \mathrm{C}$ NMR $\left(101 \mathrm{MHz}, \mathrm{CDCl}_{3}\right) \delta$ (ppm) 162.4, 151.4, 142.5, 137.5, 128.4, 127.9, 127.8, 113.9, 102.2, $99.4,84.5,84.2,73.8,72.3,70.0,53.9,36.2,34.9,25.0,24.0,23.8,14.6$.

IR (ATR): $\tilde{v}\left(\mathrm{~cm}^{-1}\right)=3434,2935,2859,1715,1665,1453,1362,1278,1211,1158,1099$.

HR-MS (ESI): $m / z$ calcd for $\mathrm{C}_{24} \mathrm{H}_{28} \mathrm{~N}_{2} \mathrm{O}_{7} \mathrm{Na}^{+}: 479.17887$; found: 479.17958 .

$[\alpha]_{\mathrm{D}}^{20}=48.8^{\circ}\left(\mathrm{c}=0.42, \mathrm{CHCl}_{3}\right)$. 


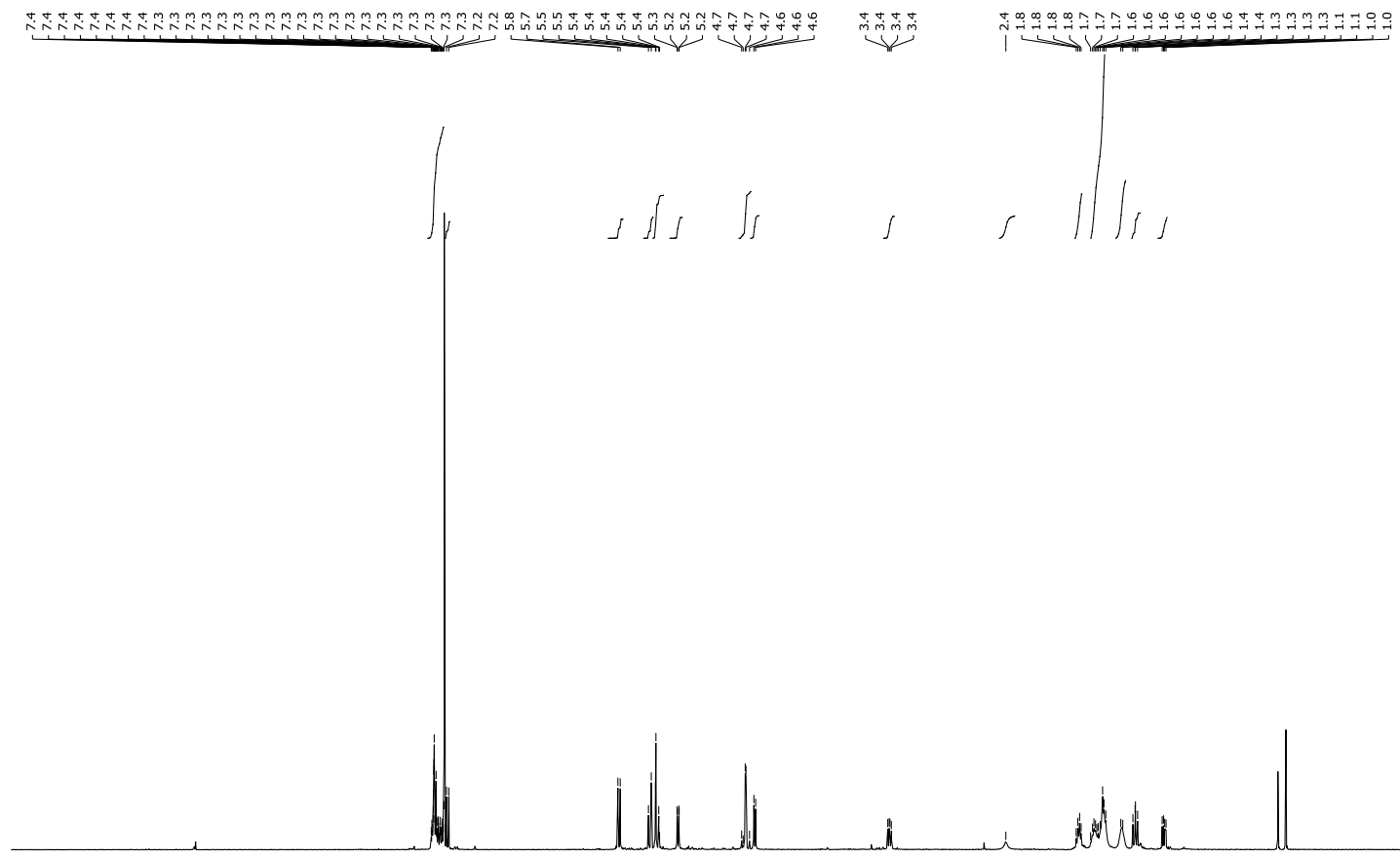

\begin{tabular}{llllllllllllllllllllllllllllll}
\hline .0 & 10.5 & 10.0 & 9.5 & 9.0 & 8.5 & 8.0 & 7.5 & 7.0 & 6.5 & 6.0 & 5.5 & $\begin{array}{c}5 \\
5(0)\end{array}$ & 4.5 & 4.0 & 3.5 & 3.0 & 2.5 & 2.0 & 1.5 & 1.0 & 0.5 & 0.0 & -0.5 & -1
\end{tabular}

Figure S21. ${ }^{1} \mathrm{H} \mathrm{NMR}\left(400 \mathrm{MHz}, \mathrm{CDCl}_{3}\right)$ spectrum of compound 9.

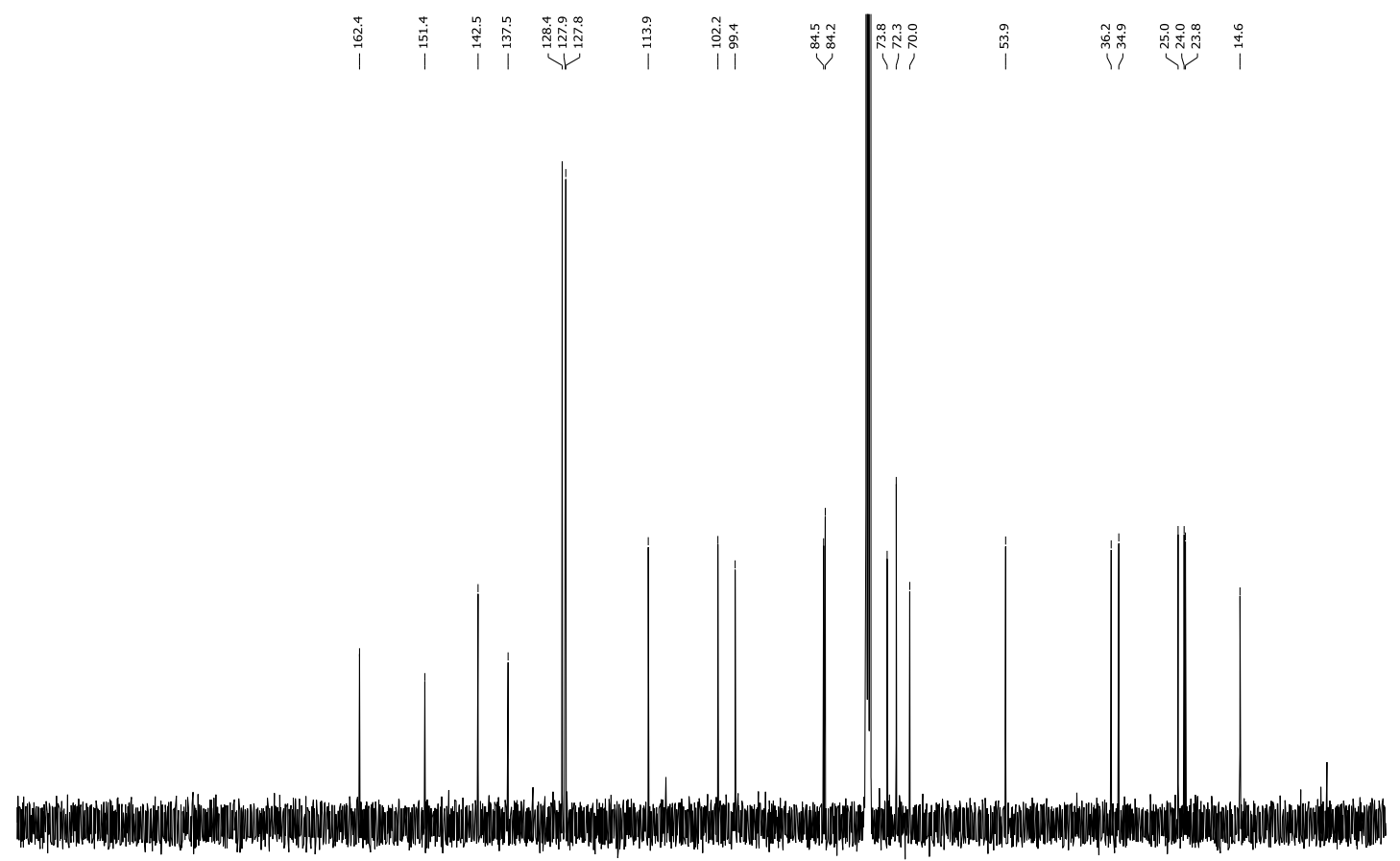

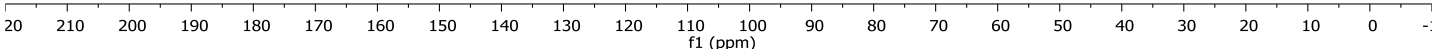

Figure S18. ${ }^{13} \mathrm{C}$ NMR (101 MHz, $\mathrm{CDCl}_{3}$ ) spectrum of compound 9. 


\section{Compound 10}

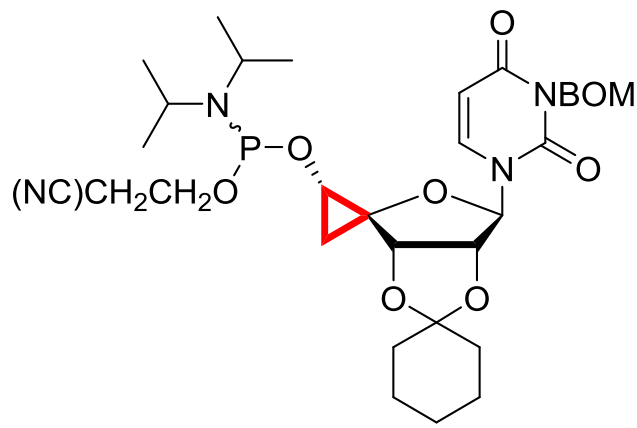

Before use compound 9 was azeotropically distilled with toluene (3x,5.00 mL). To a solution of compound 9 (1.35 g, $2.95 \mathrm{mmol}, 1.00$ eq.) in dichloromethane (30.0 mL) was added NEt'Pr $\mathrm{Pr}_{2}$ (2.51 mL, $14.8 \mathrm{mmol}, 5.00$ eq.) at $0^{\circ} \mathrm{C}$. After the dropwise addition of 2-cyanoethyl $N, N$ diisopropylchlorophosphoramidite $(1.32 \mathrm{~mL}, 5.91 \mathrm{mmol}, 2.00$ eq.) via syringe, the reaction mixture was stirred for $2 \mathrm{~h}$. After full conversion of starting material, the reaction mixture was diluted with chloroform $(30.0 \mathrm{~mL})$ and the reaction terminated by the addition of a saturated solution of $\mathrm{NaHCO}_{3}(30.0 \mathrm{~mL})$. The aqueous layer was extracted with chloroform $(3 \mathrm{x}, 20.0 \mathrm{~mL})$ and the organic layers were combined. After drying over $\mathrm{Na}_{2} \mathrm{SO}_{4}$ and filtration, the solvent was removed under reduced pressure. Column purification of the remaining oily residue afforded the desired diastereomeric mixture of phosphoramidite 10 (1.07 g, $1.63 \mathrm{mmol}, 55 \%)$ as a yellowish oil.

${ }^{31} \mathbf{P}$ NMR (162 MHz, $\left.\mathrm{CDCl}_{3}\right) \delta(\mathrm{ppm})$ 149.3, 149.2. 


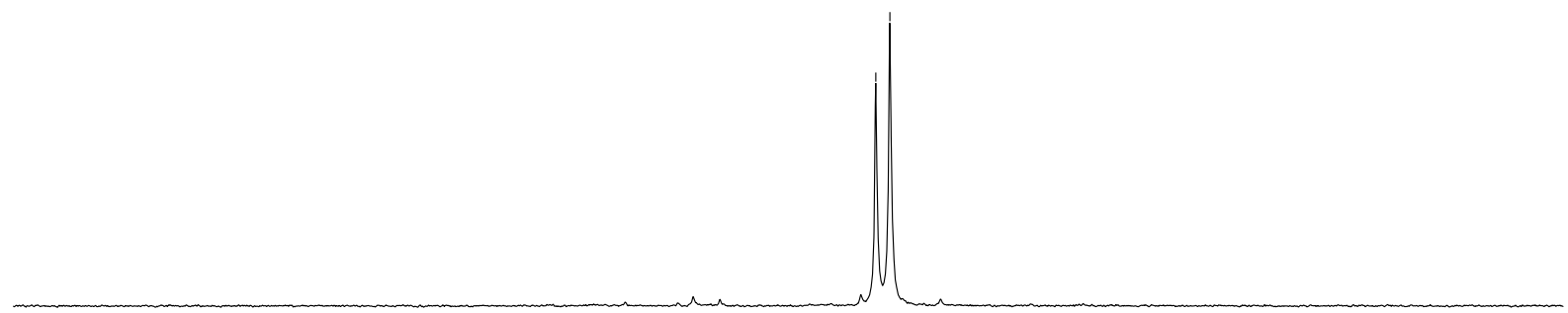

$\begin{array}{lllllllllllllllllllllllllllll}6.0 & 155.5 & 155.0 & 154.5 & 154.0 & 153.5 & 153.0 & 152.5 & 152.0 & 151.5 & 151.0 & 150.5 & 150.0 & 149.5 & 149.0 & 148.5 & 148.0 & 147.5 & 147.0 & 146.5 & 146.0 & 145.5 & 145.0 & 144.5 & 14\end{array}$ Figure S23. ${ }^{31} \mathrm{P} \mathrm{NMR}\left(162 \mathrm{MHz}, \mathrm{CDCl}_{3}\right)$ spectrum of compound 10. 


\section{Compound 11}

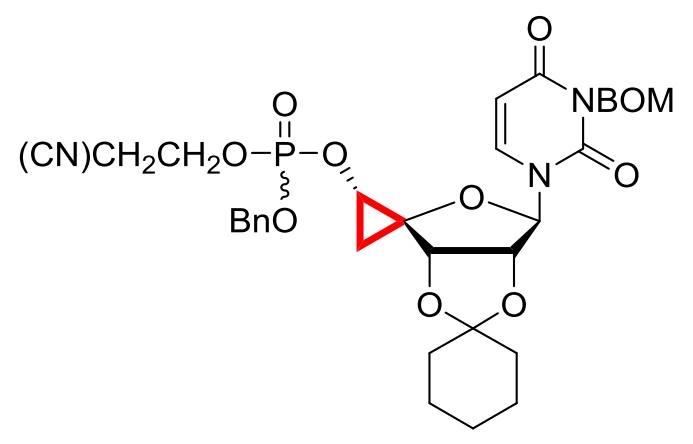

Before use compound 10 was azeotropically distilled with toluene (3x, $2.00 \mathrm{~mL})$. Phosphoramidite 10 (0.79 g, $1.20 \mathrm{mmol}, 1.00 \mathrm{eq}$.) was dissolved in acetonitrile (9.00 mL) and benzyl alcohol (0.15 mL, 1.44 mmol, 1.20 eq.) was added. Tetrazole (10.5 mL, $3.60 \mathrm{mmol}, 3.0$ eq., 3.0\% to 4.0\% $(\mathrm{w} / \mathrm{w})$ in acetonitrile) was added dropwise via syringe and the reaction mixture was stirred at room temperature for $6 \mathrm{~h}$. After consumption of all starting material the reaction mixture was cooled to $0{ }^{\circ} \mathrm{C}$ and $\mathrm{mCPBA}(0.36 \mathrm{~g}, 1.44 \mathrm{mmol}, 1.20 \mathrm{eq}$., 70\%) was added in three portions. The reaction mixture was stirred for further $0.5 \mathrm{~h}$ at $0{ }^{\circ} \mathrm{C}$ and finally diluted with dichloromethane $(20.0 \mathrm{~mL})$ and a saturated solution of $\mathrm{NaHCO}_{3}(10.0 \mathrm{~mL})$. The aqueous layer was extracted with dichloromethane $(3 \mathrm{x}, 20.0 \mathrm{~mL})$ and the combined organic phases were dried over $\mathrm{Na}_{2} \mathrm{SO}_{4}$, filtered and evaporated to dryness. Column purification $\left(\mathrm{SiO}_{2}\right.$, pentane/ethyl acetate, 5:1 to 1:1) afforded a diastereomeric mixture of phosphate 11 (0.56 g, $0.82 \mathrm{mmol}, 69 \%$ yield).

${ }^{31} \mathbf{P}$ NMR $\left(162 \mathrm{MHz}, \mathrm{CDCl}_{3}\right) \delta-1.1,-1.2$. 


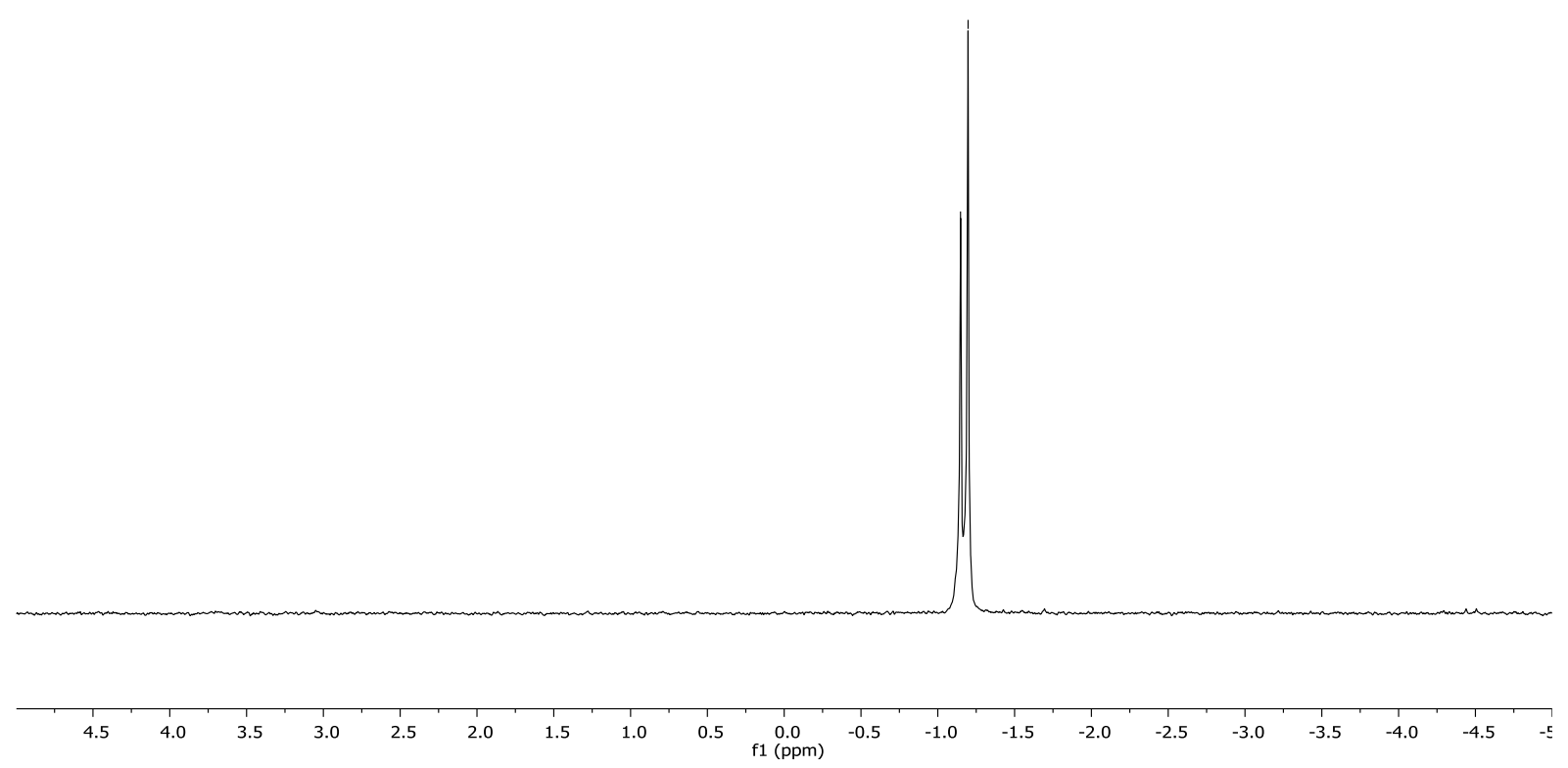

Figure S24. ${ }^{31} \mathrm{P}$ NMR (162 MHz, $\mathrm{CDCl}_{3}$ ) spectrum of compound 11. 


\section{Compound 12}

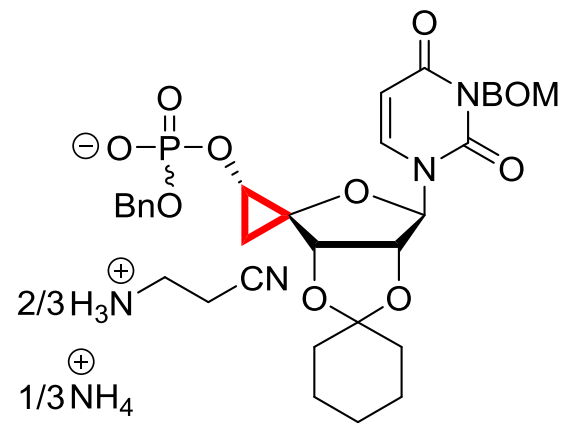

To a stirred solution of compound 11 (0.60 g, $0.88 \mathrm{mmol}, 1.00$ eq.) in dioxane $(20.0 \mathrm{~mL})$ was added the equal volume of an aqueous solution of $\mathrm{NH}_{4} \mathrm{OH}(20.0 \mathrm{~mL}, 25 \%(\mathrm{w} / \mathrm{w}))$. The reaction mixture was stirred at room temperature for $1.5 \mathrm{~h}$ and after full consumption of starting material the solvent was removed under reduced pressure. The product 12 was afforded as a mixed salt (0.60 g, $0.88 \mathrm{mmol}$, quant.). Column purification (neutralized $\mathrm{SiO}_{2}, \mathrm{DCM} / \mathrm{MeOH}, 10: 1,0.5 \% \mathrm{NEt}_{3}$ ) is not recommended for further steps as it led to diminished yields of $12 \mathbf{a}$.

${ }^{1} \mathbf{H}$ NMR $\left(300 \mathrm{MHz}, \mathrm{CDCl}_{3}\right) \delta(\mathrm{ppm}) 7.37(\mathrm{~d}, J=8.1 \mathrm{~Hz}, 1 \mathrm{H}), 7.34-7.19(\mathrm{~m}, 10 \mathrm{H}), 5.75-5.70$ $(\mathrm{m}, 2 \mathrm{H}), 5.41-5.29(\mathrm{~m}, 2 \mathrm{H}), 5.06(\mathrm{~d}, J=5.9 \mathrm{~Hz}, 1 \mathrm{H}), 4.81(\mathrm{~d}, J=6.9 \mathrm{~Hz}, 2 \mathrm{H}), 4.63(\mathrm{~s}, 2 \mathrm{H}), 4.52$ (d, $J=5.9 \mathrm{~Hz}, 1 \mathrm{H}), 3.65(\mathrm{td}, J=5.8,3.3 \mathrm{~Hz}, 1 \mathrm{H}), \delta 2.72(\mathrm{t}, J=7.4 \mathrm{~Hz}, 1.3 \mathrm{H})\left({ }^{+} \mathrm{NH}_{3} \mathrm{CH}_{2} \mathrm{CH} \mathrm{H}_{2} \mathrm{CN}\right)$, $2.50(\mathrm{t}, J=7.3 \mathrm{~Hz}, 1.3 \mathrm{H})\left({ }^{+} \mathrm{NH}_{3} \mathrm{CH}_{2} \mathrm{CH}_{2} \mathrm{CN}\right), 1.82-1.47(\mathrm{~m}, 8 \mathrm{H}), 1.39(\mathrm{~s}, 2 \mathrm{H}), 1.32-1.21(\mathrm{~m}, 2 \mathrm{H})$.

${ }^{13} \mathrm{C}$ NMR (151 MHz, $\left.\mathrm{CDCl}_{3}\right) \delta(\mathrm{ppm}) 163.1,151.0,141.3,137.4,137.3(\mathrm{~d}, J=7.6 \mathrm{~Hz}), 128.4$, 128.2, 127.8, 127.6, 127.6, 127.2, $116.4\left({ }^{+} \mathrm{NH}_{3} \mathrm{CH}_{2} \mathrm{CH}_{2} \mathrm{CN}\right), 113.8,101.5,94.4,84.1,82.8,72.1$, $71.1(\mathrm{~d}, J=7.2 \mathrm{~Hz}), 70.2,67.5(\mathrm{~d}, J=5.3 \mathrm{~Hz}), 55.3(\mathrm{~d}, J=5.8 \mathrm{~Hz}), 35.8,34.8,34.8$ $\left({ }^{+} \mathrm{NH}_{3} \mathrm{CH}_{2} \mathrm{CH}_{2} \mathrm{CN}\right), 24.7,23.7,23.5,15.8\left({ }^{+} \mathrm{NH}_{3} \mathrm{CH}_{2} \mathrm{CH}_{2} \mathrm{CN}\right)$, 13.6.

${ }^{31} \mathbf{P}$ NMR $\left(162 \mathrm{MHz}, \mathrm{CDCl}_{3}\right) \delta(\mathrm{ppm})-1.0$.

IR (ATR): $\tilde{v}\left(\mathrm{~cm}^{-1}\right)=2923,2855,2323,1716,1666,1492,1456,1368,1214,1156,1082$.

HR-MS (ESI): $m / z$ calcd for $\mathrm{C}_{31} \mathrm{H}_{35} \mathrm{~N}_{2} \mathrm{O}_{10} \mathrm{P}\left(\mathrm{C}_{3} \mathrm{H}_{7} \mathrm{~N}_{2}\right)^{+}: 697.26331$; found: 697.26325 .

$[\alpha]_{\mathrm{D}}^{20}=33.2^{\circ}\left(\mathrm{c}=0.81, \mathrm{CHCl}_{3}\right)$. 

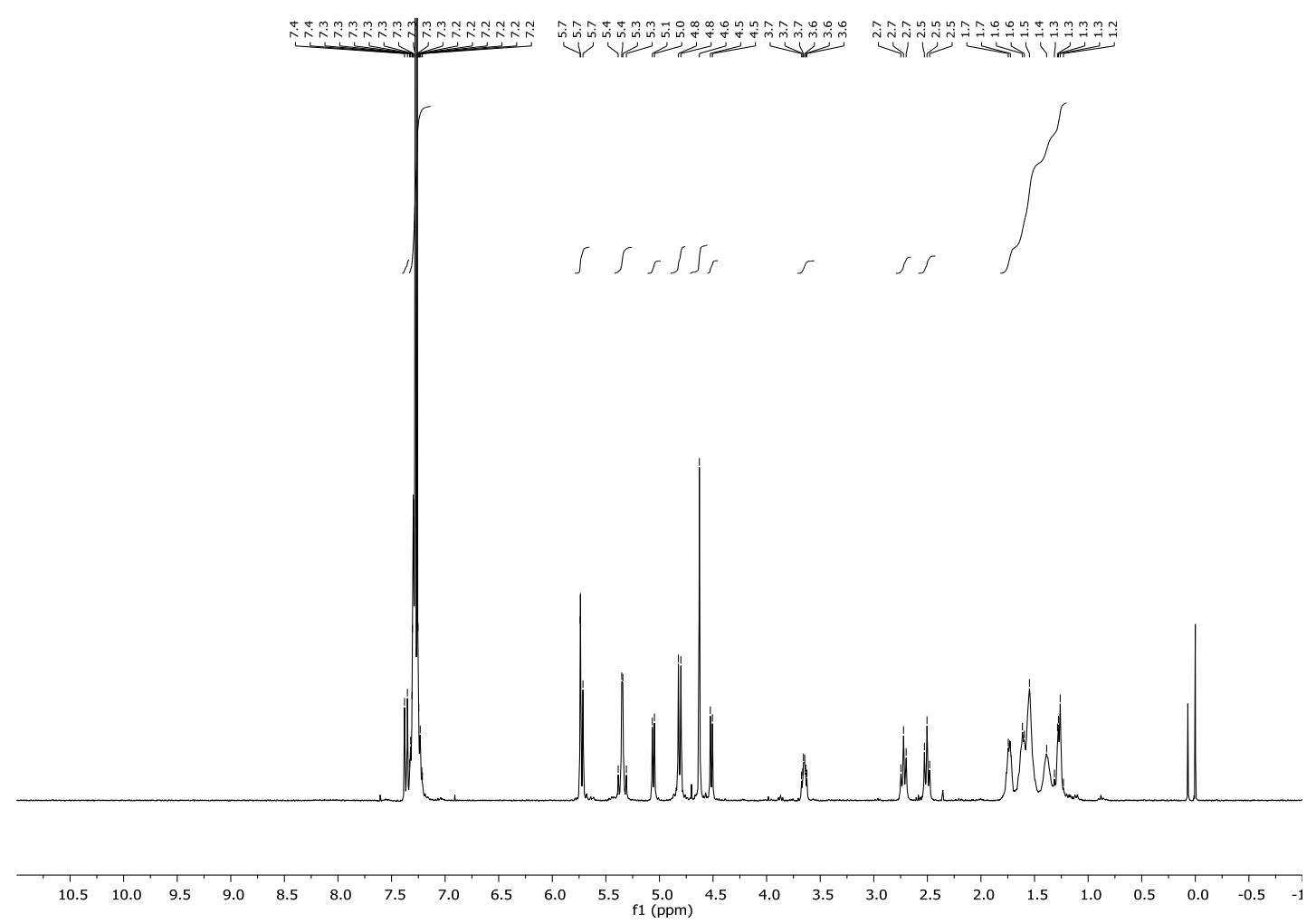

Figure S25. ${ }^{1} \mathrm{H} \mathrm{NMR}\left(300 \mathrm{MHz}, \mathrm{CDCl}_{3}\right)$ spectrum of compound 12.
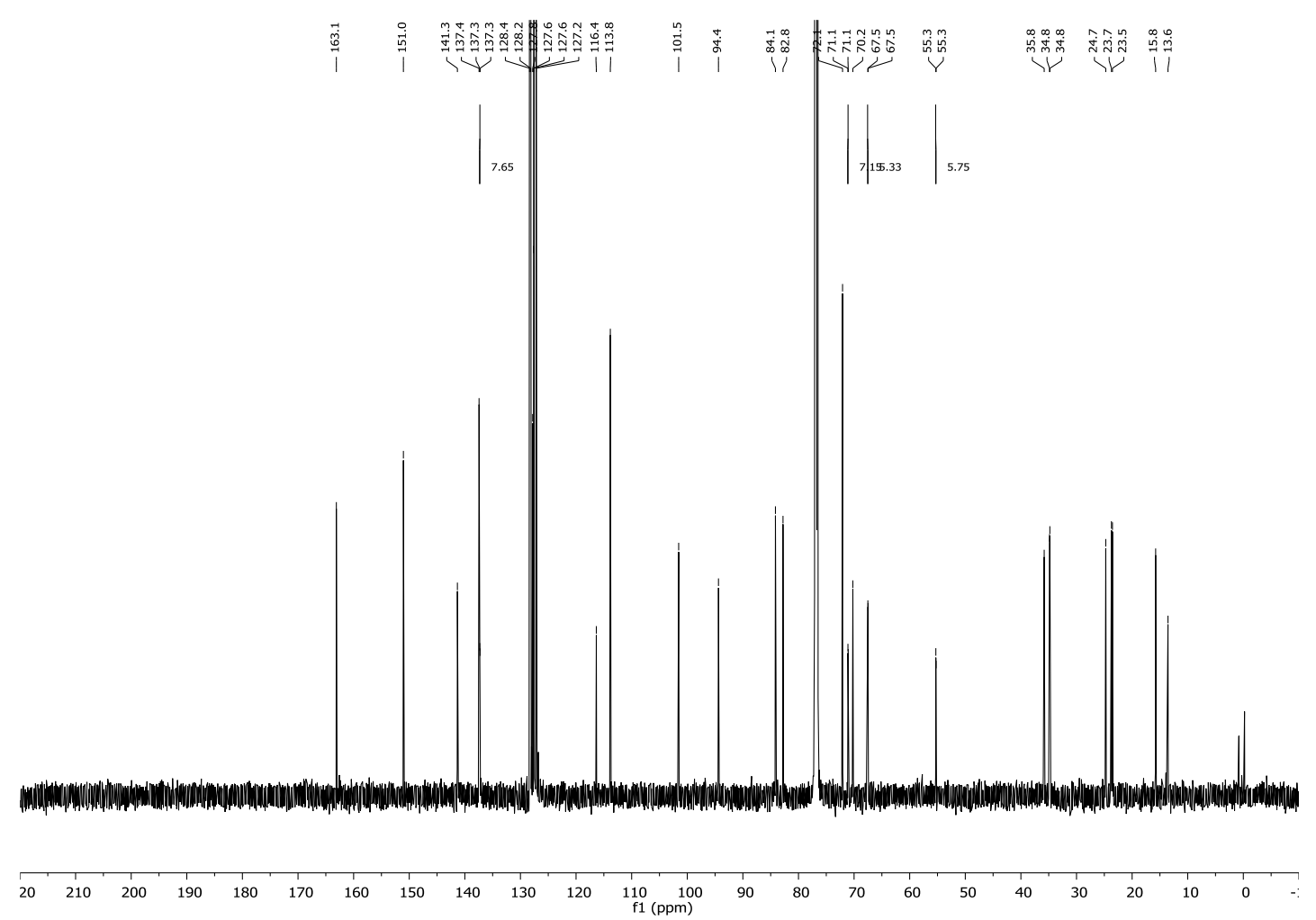

Figure S26. ${ }^{1} \mathrm{H}$ NMR (151 MHz, $\mathrm{CDCl}_{3}$ ) spectrum of compound 12. 


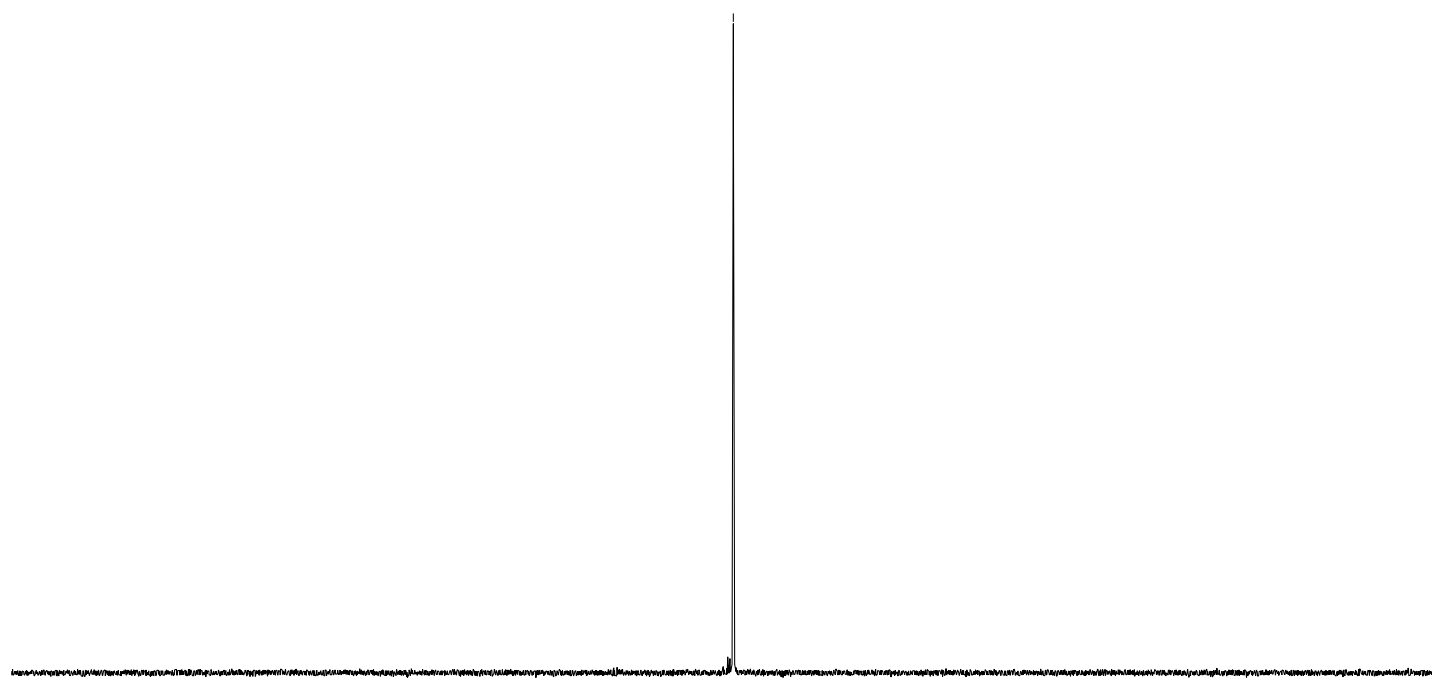

$\begin{array}{llllllllllllllllllllllllllll}55 & 50 & 45 & 40 & 35 & 30 & 25 & 20 & 15 & 10 & 5 & 0 & -5 & -10 & -15 & -20 & -25 & -30 & -35 & -40 & -45 & -50 & -55 & -1\end{array}$

Figure S27. ${ }^{31} \mathrm{P}$ NMR (162 MHz, $\left.\mathrm{CDCl}_{3}\right)$ spectrum of compound 12. 


\section{Compound 12a}

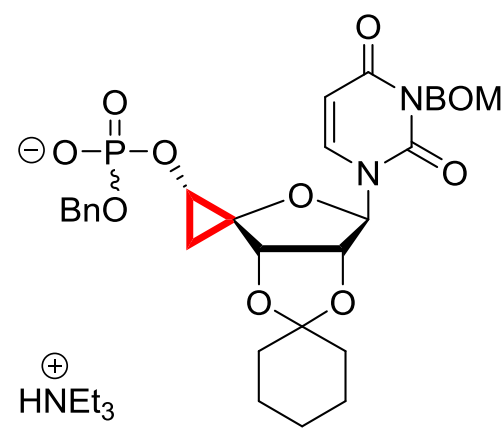

${ }^{1}$ H NMR (300 MHz, CD 30 D) $\delta(p p m) 7.64(\mathrm{~d}, J=8.1 \mathrm{~Hz}, 1 \mathrm{H}), 7.30-7.05(\mathrm{~m}, 10 \mathrm{H}), 5.72(\mathrm{~d}, J=$ $0.6 \mathrm{~Hz}, 1 \mathrm{H}$ ), 5.61 (d, J = 8.1 Hz, 1H), 5.30 (d, J=0.8 Hz, 2H), 4.94 (dd, J=6.0, $0.9 \mathrm{~Hz}, 1 \mathrm{H}$ ), 4.55 (s, 2H), $4.48(\mathrm{~d}, J=6.0 \mathrm{~Hz}, 1 \mathrm{H}), 3.60(\mathrm{td}, J=6.0,2.9 \mathrm{~Hz}, 1 \mathrm{H}), 3.10(\mathrm{q}, J=6.9 \mathrm{~Hz}, 6 \mathrm{H}), 1.72-$ $1.62(\mathrm{~m}, 2 \mathrm{H}), 1.60-1.46(\mathrm{~m}, 6 \mathrm{H}), 1.38-1.28(\mathrm{~m}, 2 \mathrm{H}), 1.21(\mathrm{~m}, 11 \mathrm{H})$.

${ }^{13} \mathrm{C}$ NMR (151 MHz, CD $\left.{ }_{3} \mathrm{OD}\right) \delta(\mathrm{ppm}) 165.1,152.6,143.2,139.5$ (d, J = 8.3 Hz), 129.4, 129.3, 128.7, 128.7, 128.6, 128.4, 114.8, 102.2, 96.0, 86.4, 84.6, 73.3 (d, J = 7.4 Hz), 73.2, 71.7, 68.5 (d, $J=5.5 \mathrm{~Hz}), 56.9$ (d, $J=5.3 \mathrm{~Hz}$ ), 37.2, 36.2, 26.2, 25.1, 24.9, 14.8, 9.2.

${ }^{31} \mathbf{P}$ NMR (122 MHz, CD $\left.\mathrm{CD}_{3} \mathrm{O}\right) \delta(\mathrm{ppm}) 0.4$.

IR (ATR): $\tilde{v}\left(\mathrm{~cm}^{-1}\right)=3095,3063,3030,2928,2856,1713,1665,1452,1363,1258,1217,1085$, 1020.

HR-MS (ESI): $m / z$ calcd for $\mathrm{C}_{31} \mathrm{H}_{34} \mathrm{~N}_{2} \mathrm{O}_{10} \mathrm{PNa}_{2}{ }^{+}: 671.17410$; found: 671.17396 . $[\alpha]_{\mathrm{D}}^{20}=-21.1^{\circ}\left(\mathrm{c}=0.18, \mathrm{CD}_{3} \mathrm{OD}\right)$ 


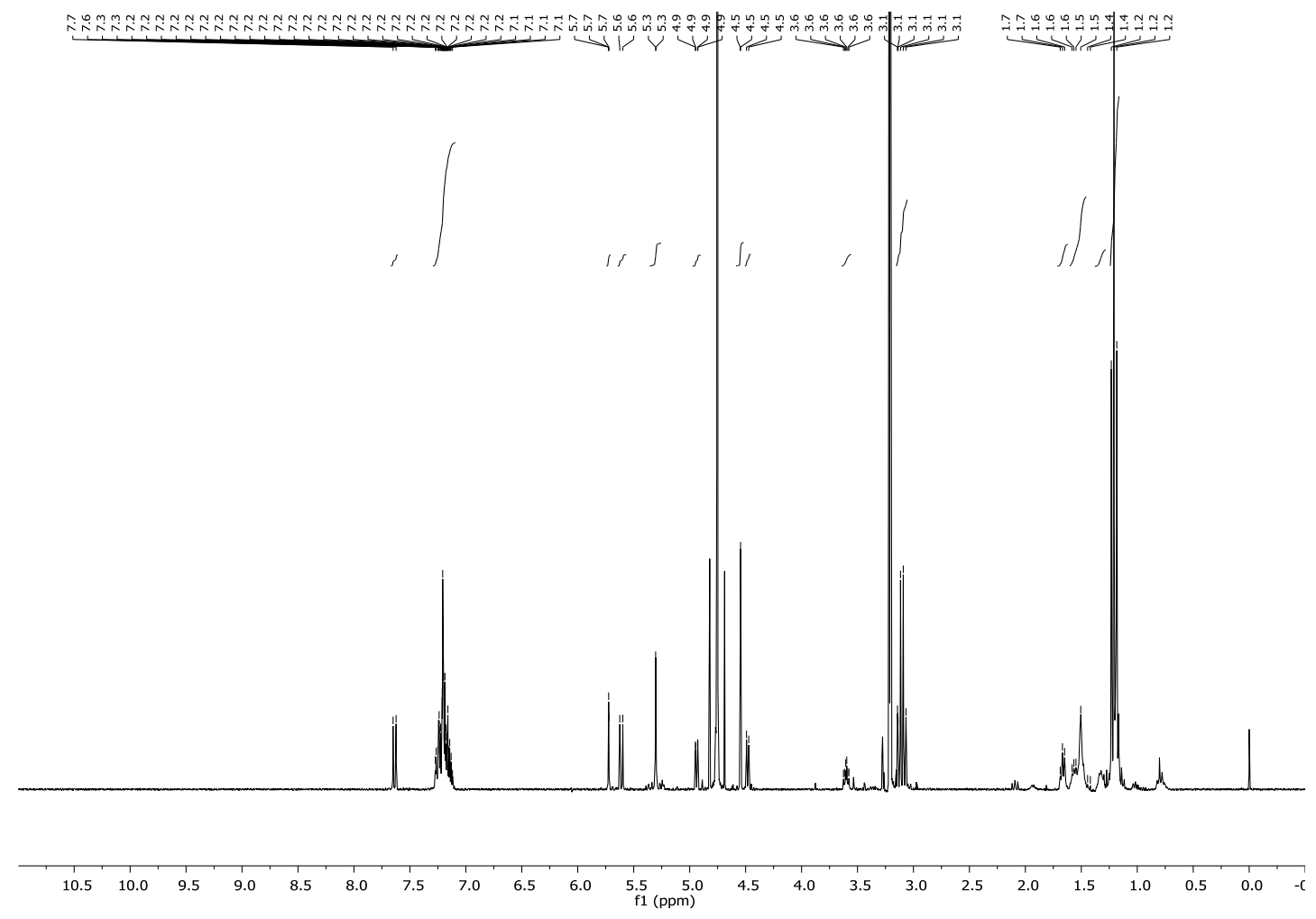

Figure S28. ${ }^{1} \mathrm{H}$ NMR (300 MHz, $\left.\mathrm{CD}_{3} \mathrm{OD}\right)$ spectrum of compound 12a.

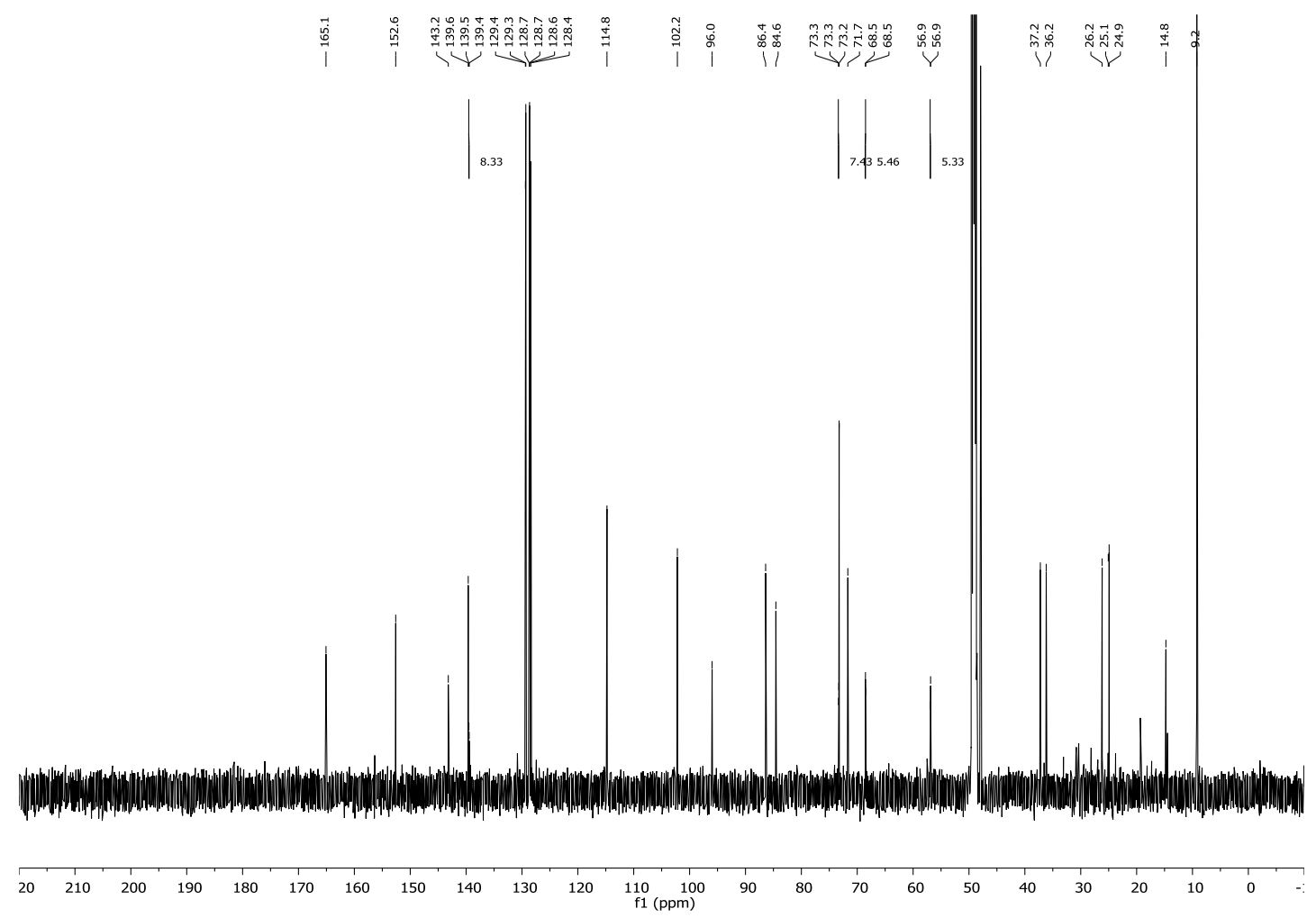

Figure S29. ${ }^{13} \mathrm{C}$ NMR (151 MHz, $\left.\mathrm{CD}_{3} \mathrm{OD}\right)$ spectrum of compound 12a. 


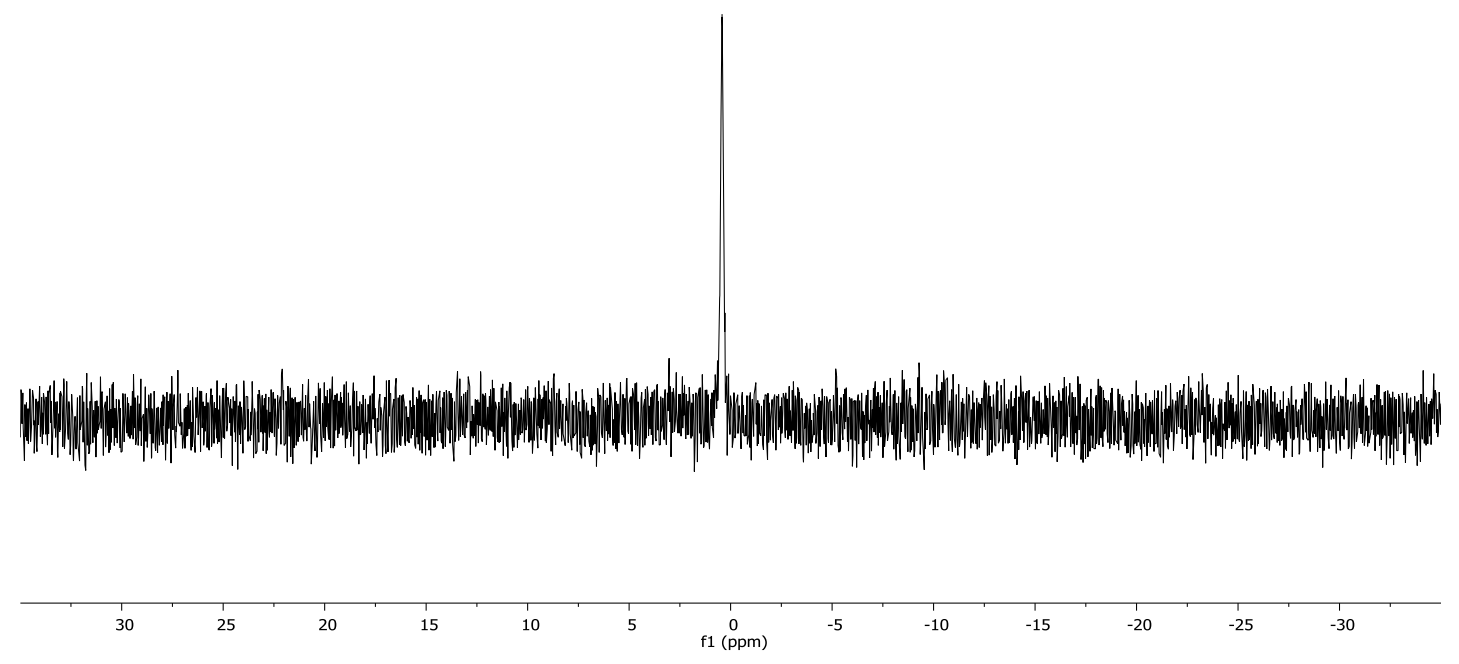

Figure S30. ${ }^{31} \mathrm{P}$ NMR (122 MHz, $\left.\mathrm{CD}_{3} \mathrm{OD}\right)$ spectrum of compound 12a. 


\section{Compound 13}

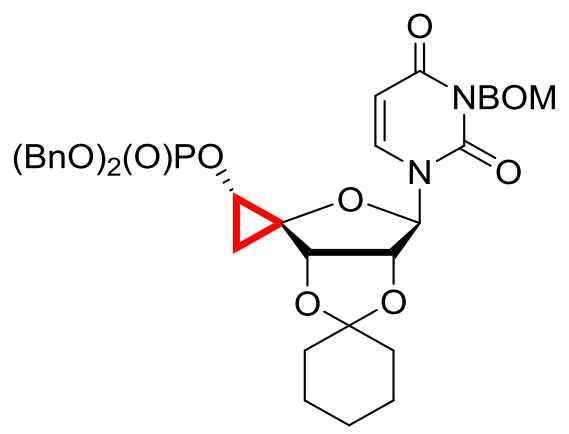

To a stirred solution of compound 12 (43.4 mg, $69.3 \mu \mathrm{mol}, 1.00$ eq.) in DMSO (1.40 mL) was added tetrabutylammonium iodide $(5.10 \mathrm{mg}, 13.8 \mu \mathrm{mol}, 0.20$ eq.), caesium carbonate (45.0 mg, $138 \mu \mathrm{mol}, 2.00$ eq.) and benzyl bromide $(41.0 \mu \mathrm{L}, 345 \mu \mathrm{mol}, 5.00$ eq.). The reaction mixture was heated to $50{ }^{\circ} \mathrm{C}$, stirred for $12 \mathrm{~h}$ and terminated by the addition of ethyl acetate $(10 \mathrm{~mL}) . \mathrm{A}$ saturated aqueous solution of $\mathrm{NaHCO}_{3}(5.00 \mathrm{~mL})$ was added and the aqueous layer extracted with ethyl acetate $(3 x, 5.00 \mathrm{~mL})$. The combined organic layers were dried over $\mathrm{Na}_{2} \mathrm{SO}_{4}$, filtered and the solvent removed in vacuum. After column purification $\left(\mathrm{SiO}_{2}\right.$, pentane/ethyl acetate, 2:1 to $1: 1)$ the product 13 (16.6 mg, $23.2 \mu \mathrm{mol}, 33 \%)$ was afforded as a colorless oil.

${ }^{1} \mathrm{H}$ NMR $\left(300 \mathrm{MHz}, \mathrm{CDCl}_{3}\right) \delta(\mathrm{ppm}) 7.42-7.18(\mathrm{~m}, 15 \mathrm{H}), 7.18(\mathrm{~d}, J=8.1 \mathrm{~Hz}, 1 \mathrm{H}), 5.61(\mathrm{~d}, J=$ $8.0 \mathrm{~Hz}, 1 \mathrm{H}), 5.57(\mathrm{~s}, 1 \mathrm{H}), 5.37(\mathrm{~d}, J=9.8 \mathrm{~Hz}, 1 \mathrm{H}), 5.32(\mathrm{~d}, J=9.8 \mathrm{~Hz}, 1 \mathrm{H}), 5.23(\mathrm{dd}, J=6.0,0.9$ $\mathrm{Hz}, 1 \mathrm{H}), 4.98-4.88(\mathrm{~m}, 4 \mathrm{H}), 4.63(\mathrm{~s}, 2 \mathrm{H}), 4.61(\mathrm{~d}, J=6.0 \mathrm{~Hz}, 1 \mathrm{H}), 3.77$ (ddd, $J=6.4,5.1,2.6$ $\mathrm{Hz}, 1 \mathrm{H}), 1.76(\mathrm{dd}, J=7.6,4.2 \mathrm{~Hz}, 2 \mathrm{H}), 1.64-1.52(\mathrm{~m}, 6 \mathrm{H}), 1.45-1.35(\mathrm{~m}, 4 \mathrm{H})$.

${ }^{13} \mathrm{C}$ NMR (126 MHz, $\left.\mathrm{CDCl}_{3}\right) \delta(\mathrm{ppm}) 162.5,151.1,141.5,137.9,135.5$ (d, J = 6.9 Hz), 135.4 (d, $J=6.9 \mathrm{~Hz}), 128.6,128.6,128.6,128.2,127.9,127.9,127.7,127.6,114.1,102.2,96.3,84.5,83.3$, 72.2, 71.6 (d, $J=7.3 \mathrm{~Hz}), 70.2,69.5$ (d, $J=3.6 \mathrm{~Hz}), 69.5(\mathrm{~d}, J=3.7 \mathrm{~Hz}), 56.9$ (d, $J=5.5 \mathrm{~Hz})$, 36.0, 35.0, 24.9, 23.9, 23.7, 14.1 (d, J=1.4 Hz).

${ }^{31} \mathbf{P}$ NMR $\left(203 \mathrm{MHz}, \mathrm{CDCl}_{3}\right) \delta(\mathrm{ppm})-0.5$.

IR (ATR): $\tilde{v}\left(\mathrm{~cm}^{-1}\right)=3089,3065,3033,2937,2860,1719,1670,1497,1451,1407,1357,1262$, 1215, 1160, 1077, 1013.

HR-MS (ESI): $m / z$ calcd for $\mathrm{C}_{38} \mathrm{H}_{41} \mathrm{~N}_{2} \mathrm{O}_{10} \mathrm{PNa}^{+}:$739.23910; found: 739.23984.

$[\alpha]_{\mathrm{D}}^{20}=97.8^{\circ}\left(\mathrm{c}=0.77, \mathrm{CHCl}_{3}\right)$. 

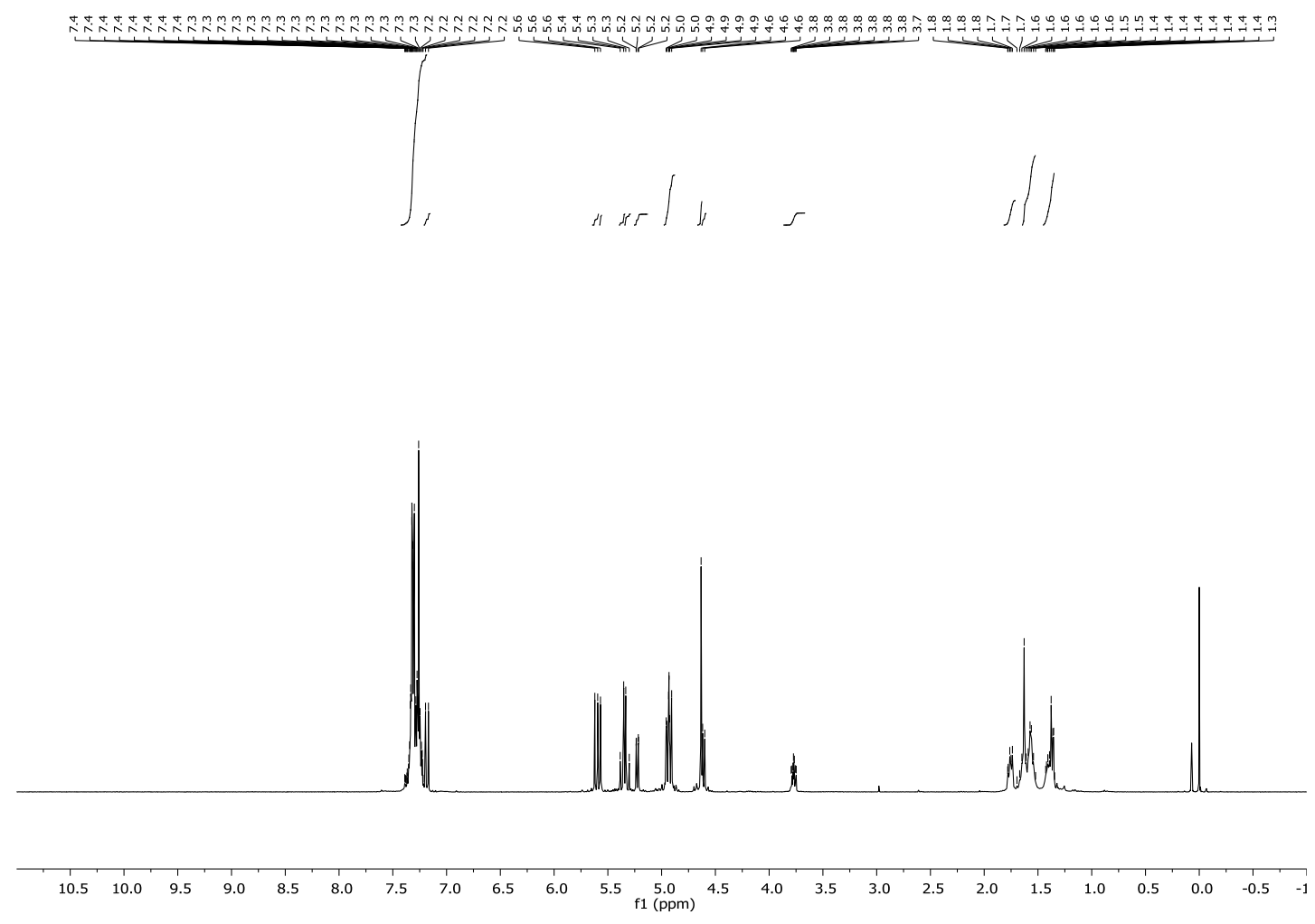

Figure S31. ${ }^{1} \mathrm{H} \mathrm{NMR}\left(300 \mathrm{MHz}, \mathrm{CDCl}_{3}\right)$ spectrum of compound 13.

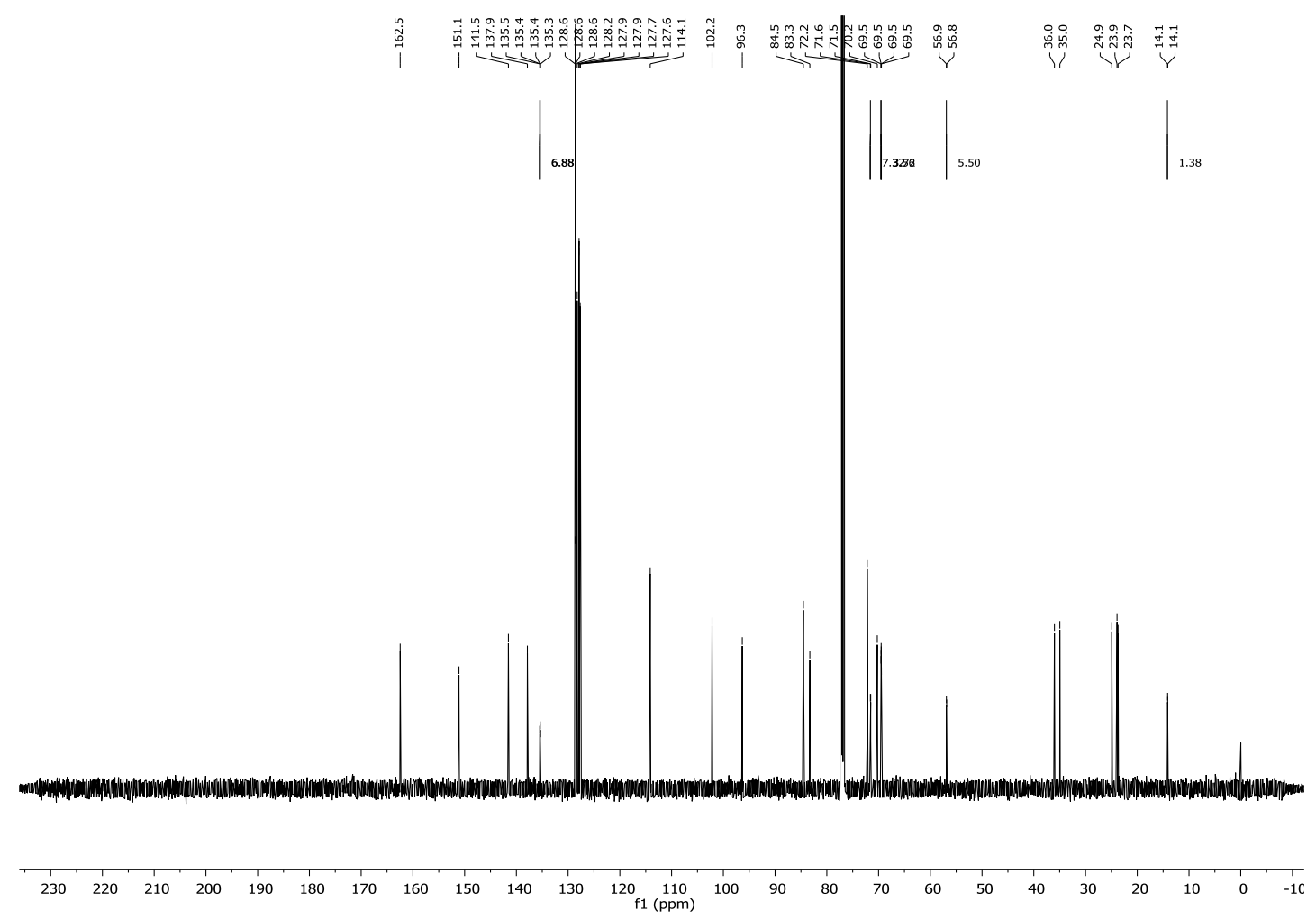

Figure S32. ${ }^{13} \mathrm{C}$ NMR (126 MHz, $\mathrm{CDCl}_{3}$ ) spectrum of compound 13. 


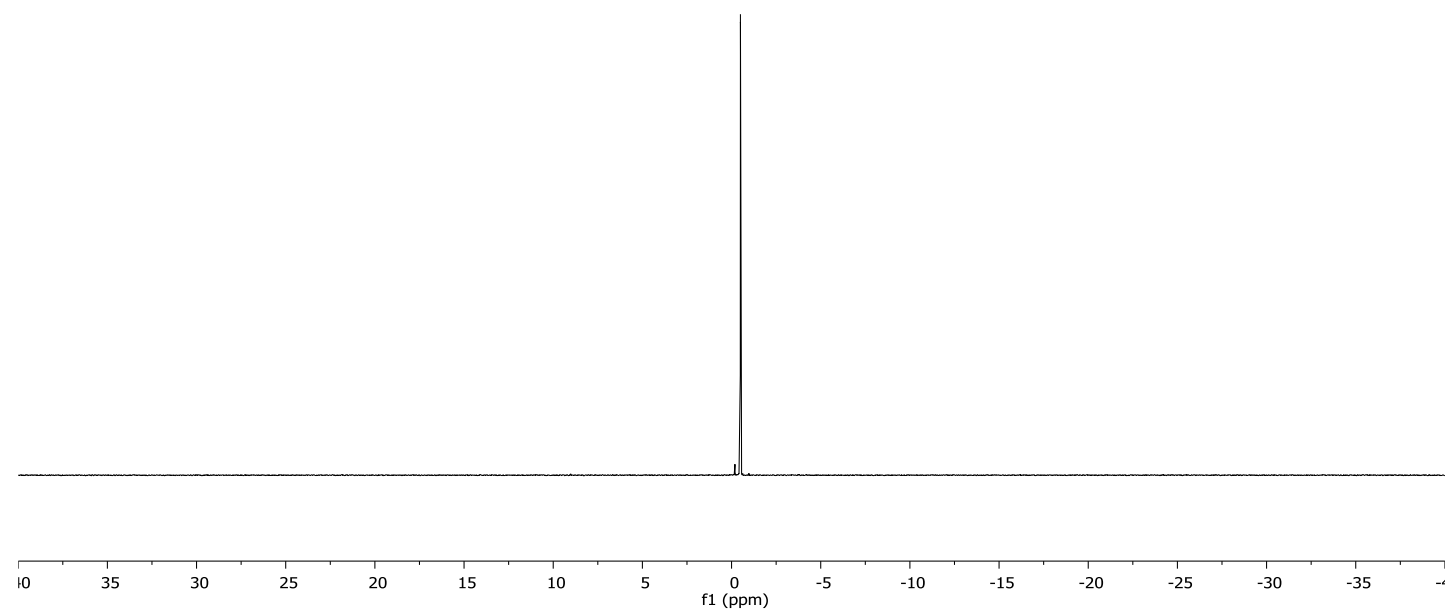

Figure S33. ${ }^{31} \mathrm{P}$ NMR (203 MHz, $\left.\mathrm{CDCl}_{3}\right)$ spectrum of compound 13. 


\section{Compound 14}

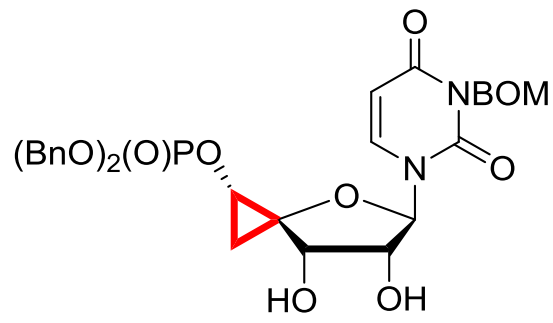

Compound 13 (0.14 g, $0.20 \mathrm{mmol}, 1.00$ eq.) was dissolved in a methanol (15.0 mL) and $\mathrm{HCl}$ in dioxane (4.00 mL, $16.0 \mathrm{mmol}, 80.0$ eq., $4.00 \mathrm{M}$ ) was added via syringe. After stirring for $10 \mathrm{~h}$ at ambient temperature the reaction mixture was diluted with dichloromethane $(50.0 \mathrm{~mL})$ and a saturated aqueous solution of $\mathrm{NaHCO}_{3}(20.0 \mathrm{~mL})$ was added. The aqueous phase was extracted with dichloromethane $(3 \mathrm{x}, 20.0 \mathrm{~mL})$, the combined organic phases were dried over $\mathrm{Na}_{2} \mathrm{SO}_{4}$ and evaporated to dryness. Purification by column chromatography $\left(\mathrm{SiO}_{2}, \mathrm{DCM} / \mathrm{MeOH}, 20: 1\right.$ to 10:1) afforded the phosphorylated syn-diol 14 (39.9 mg, $0.13 \mathrm{mmol}, 31 \%$ yield) as a colorless solid.

${ }^{1} \mathbf{H}$ NMR $\left(600 \mathrm{MHz}, \mathrm{CDCl}_{3}\right) \delta(\mathrm{ppm}) 7.73(\mathrm{~d}, J=8.2 \mathrm{~Hz}, 1 \mathrm{H}), 7.49-7.22(\mathrm{~m}, 13 \mathrm{H}), 5.81(\mathrm{~d}, J=$ $5.6 \mathrm{~Hz}, 1 \mathrm{H}), 5.54(\mathrm{~d}, J=8.3 \mathrm{~Hz}, 1 \mathrm{H}), 5.51-5.36(\mathrm{~m}, 2 \mathrm{H}), 5.08$ (dd, J=11.6, $8.8 \mathrm{~Hz}, 1 \mathrm{H}), 5.06-$ $5.01(\mathrm{~m}, 2 \mathrm{H}), 4.99(\mathrm{dd}, J=11.6,8.3 \mathrm{~Hz}, 1 \mathrm{H}), 4.69(\mathrm{~s}, 2 \mathrm{H}), 4.40(\mathrm{~d}, J=2.9 \mathrm{~Hz}, 1 \mathrm{H}), 4.17$ (td, $J=$ 5.4, $2.4 \mathrm{~Hz}, 1 \mathrm{H}$ ), 3.72 (d, J=5.0 Hz, 1H), 3.70 (ddd, J=7.5, 4.5, $2.9 \mathrm{~Hz}, 1 \mathrm{H}$ ), 3.06 (s, $1 \mathrm{H}), 1.36$ (dd, $J=9.2,7.7 \mathrm{~Hz}, 1 \mathrm{H}), 1.30(\mathrm{dd}, J=9.2,4.5 \mathrm{~Hz}, 1 \mathrm{H})$.

${ }^{13} \mathrm{C}$ NMR (126 MHz, $\left.\mathrm{CDCl}_{3}\right) \delta(\mathrm{ppm}) 162.3,152.3,138.6,137.6,135.2(\mathrm{~d}, J=6.3 \mathrm{~Hz}), 135.1$ (d, $J=6.9 \mathrm{~Hz}), 128.9,128.7,128.7,128.3,128.1,128.1$, 127.8, 127.7, 101.9, 91.1, 73.2, 72.4, 70.3, $69.9(\mathrm{~d}, J=5.8 \mathrm{~Hz}), 68.8(\mathrm{~d}, J=4.4 \mathrm{~Hz}), 55.1$ (d, $J=5.0 \mathrm{~Hz}), 12.7$ (d, J=4.9 Hz).

${ }^{31}$ P NMR $\left(203 \mathrm{MHz}, \mathrm{CDCl}_{3}\right) \delta(\mathrm{ppm})-0.1(\mathrm{pd}, J=8.6,2.7 \mathrm{~Hz})$.

IR (ATR): $\tilde{v}\left(\mathrm{~cm}^{-1}\right)=3389,3091,3065,3033,2955,2925,2856,1716,1666,1497,1457,1412$, 1358, 1254, 1215, 1126, 1053, 1016.

HR-MS (ESI): $m / z$ calcd for $\mathrm{C}_{32} \mathrm{H}_{33} \mathrm{~N}_{2} \mathrm{O}_{10} \mathrm{PNa}^{+}: 659.17650$; found: 659.17656 .

$[\alpha]_{\mathrm{D}}^{20}=24.9^{\circ}\left(\mathrm{c}=0.89, \mathrm{CHCl}_{3}\right)$. 


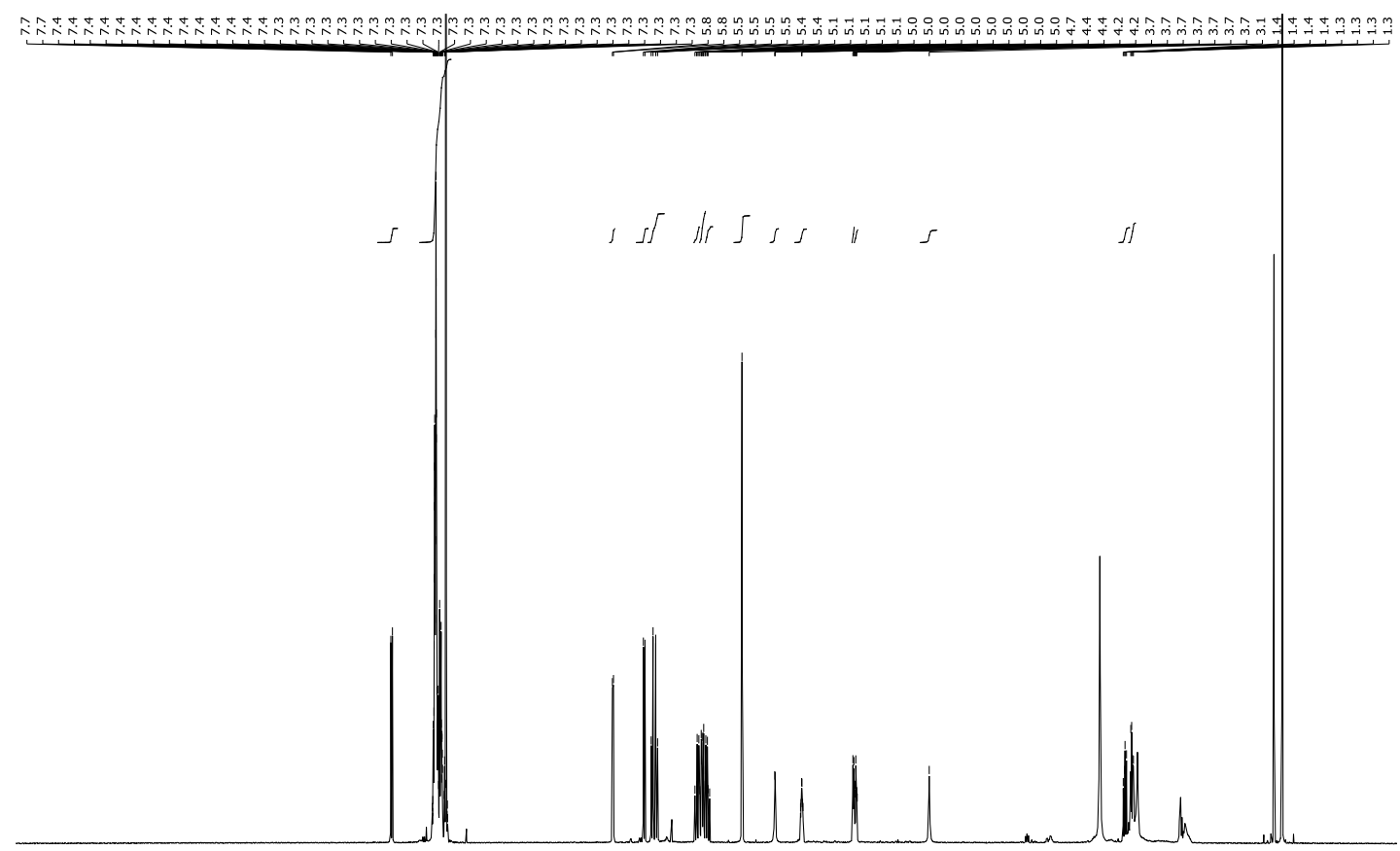

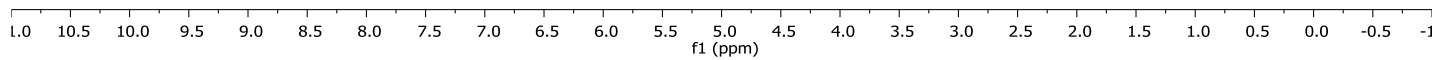

Figure S34. ${ }^{1} \mathrm{H} \mathrm{NMR}\left(600 \mathrm{MHz}, \mathrm{CDCl}_{3}\right)$ spectrum of compound 14.
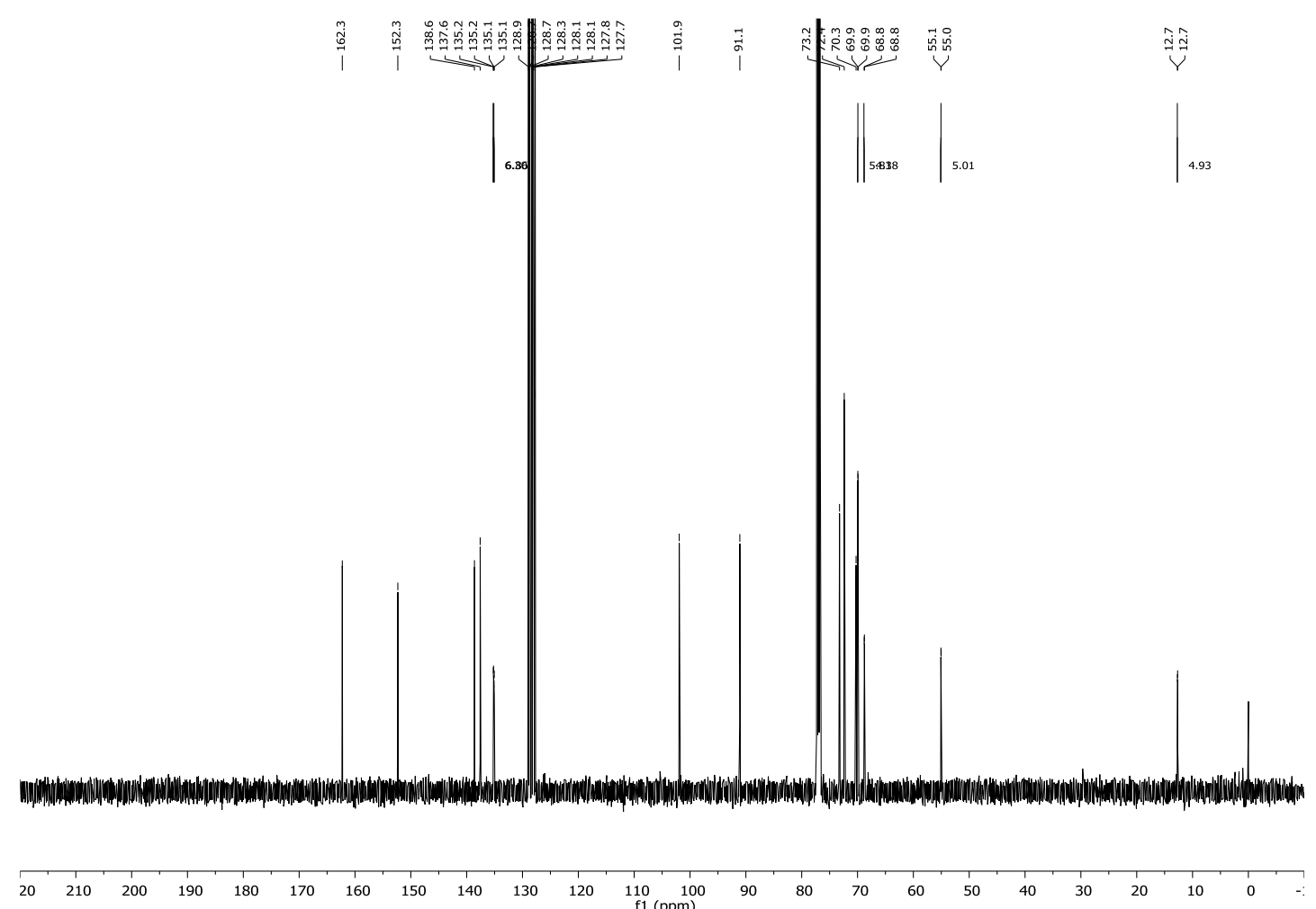

Figure S35. ${ }^{13} \mathrm{C}$ NMR (126 MHz, $\left.\mathrm{CDCl}_{3}\right)$ spectrum of compound 14. 


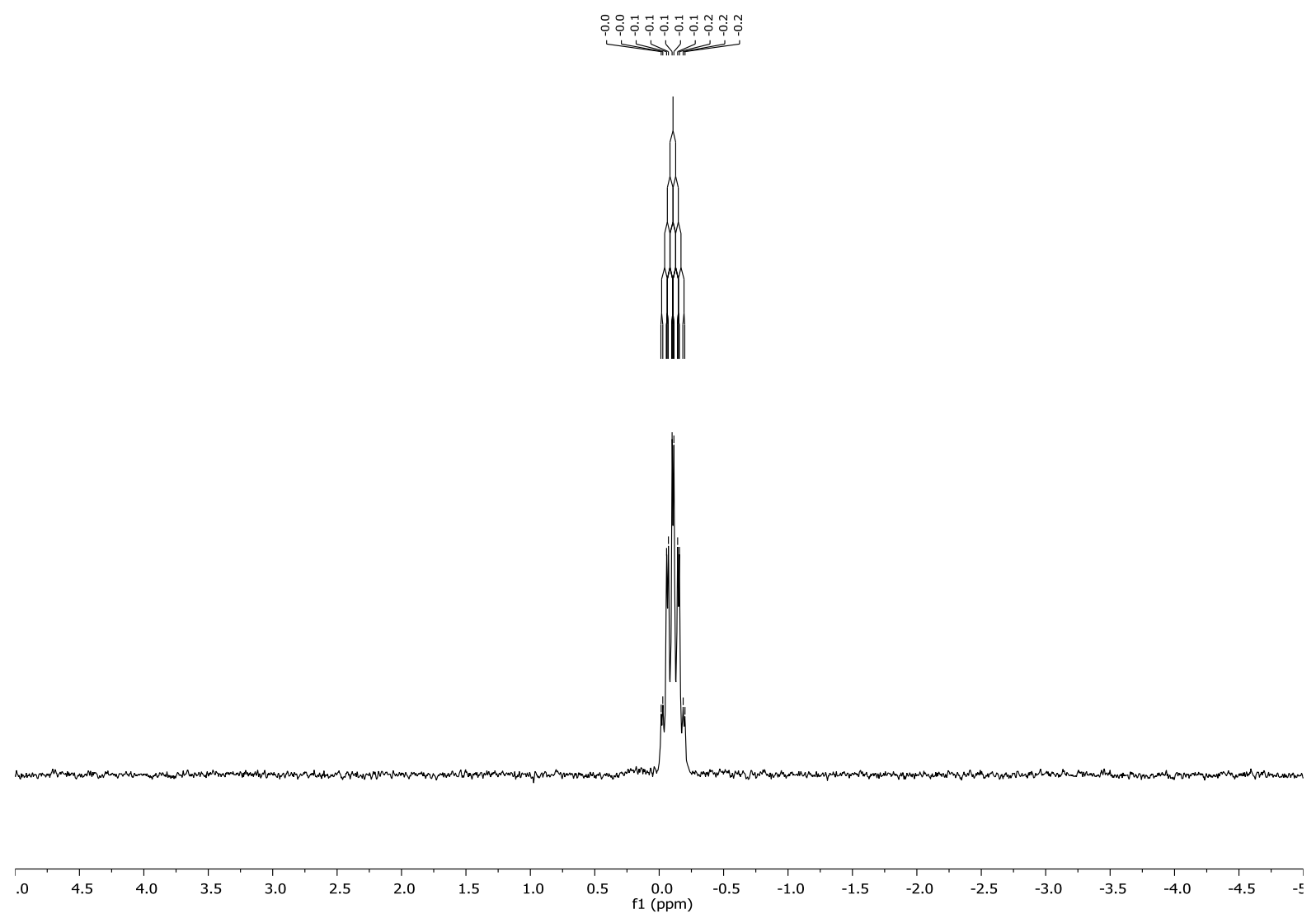

Figure S36. ${ }^{31} \mathrm{P}$ NMR (203 MHz, $\mathrm{CDCl}_{3}$ ) spectrum of compound 14 (no ${ }^{1} \mathrm{H}$ decoupling). 


\section{Compound 15}

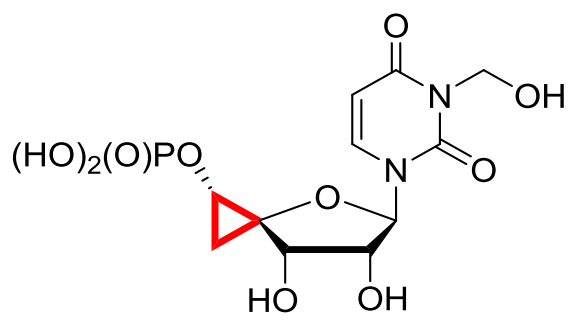

Under an argon atmosphere, derivative 14 (32.3 mg, $50.7 \mu \mathrm{mol}, 1.00$ eq.) was dissolved in methanol $(2.00 \mathrm{~mL}$ ). Palladium on charcoal (10 wt.\% on activated carbon, $11.0 \mathrm{mg}, 10.3 \mu \mathrm{mol}$, 0.20 eq.) was added and the argon atmosphere exchanged by a hydrogen atmosphere. The suspension was stirred at room temperature for $5 \mathrm{~h}$ and the reaction mixture finally filtered through Celite $\AA 545$. The Celite $\AA$ plug was rinsed with methanol $(3 x, 2.00 \mathrm{~mL})$ and evaporation of the solvent afforded phosphate 15 (18.6 mg, $50.7 \mu \mathrm{mol}$, quant) as a colorless foam.

${ }^{1} \mathrm{H}$ NMR $\left(600 \mathrm{MHz}, \mathrm{D}_{2} \mathrm{O}\right) \delta(\mathrm{ppm}) 7.93(\mathrm{~d}, J=8.2 \mathrm{~Hz}, 1 \mathrm{H}), 6.28(\mathrm{~d}, J=6.9 \mathrm{~Hz}, 1 \mathrm{H}), 6.02(\mathrm{~d}, J=$ $8.2 \mathrm{~Hz}, 1 \mathrm{H}), 5.42(\mathrm{~d}, J=10.4 \mathrm{~Hz}, 1 \mathrm{H}), 5.40(\mathrm{~d}, J=10.4 \mathrm{~Hz}, 1 \mathrm{H}), 4.67$ (dd, $J=6.9,4.6 \mathrm{~Hz}, 1 \mathrm{H})$, $3.96(\mathrm{~d}, J=4.6 \mathrm{~Hz}, 1 \mathrm{H}), 3.80(\mathrm{dt}, J=7.7,3.9 \mathrm{~Hz}, 1 \mathrm{H}), 1.36$ (dd, $J=8.9,7.5 \mathrm{~Hz}, 1 \mathrm{H}), 1.19$ (dd, $J$ $=8.9,4.4 \mathrm{~Hz}, 1 \mathrm{H})$.

${ }^{13} \mathrm{C}$ NMR $\left(151 \mathrm{MHz}, \mathrm{D}_{2} \mathrm{O}\right) \delta(\mathrm{ppm})$ 167.6, 155.0, 143.4, 105.6, 91.4, 78.1, 76.5, 71.7 (d, J = 5.9 $\mathrm{Hz}), 67.2,56.7$ (d, $J=4.7 \mathrm{~Hz}), 15.4(\mathrm{~d}, J=2.5 \mathrm{~Hz})$.

${ }^{31}$ P NMR (203 MHz, D 20$) \delta(p p m) ~ 0.8$.

IR (ATR): $\tilde{v}\left(\mathrm{~cm}^{-1}\right)=3279,2955,1701,1665,1559,1469,1411,1299,1247,1202,1078$.

HR-MS (ESI): $m / z$ calcd for $\mathrm{C}_{11} \mathrm{H}_{14} \mathrm{~N}_{2} \mathrm{O}_{10} \mathrm{P}^{-:}: 365.03915$; found: 365.03988 .

$[\alpha]_{\mathrm{D}}^{20}=20.8^{\circ}\left(\mathrm{c}=0.4, \mathrm{H}_{2} \mathrm{O}\right)$. 


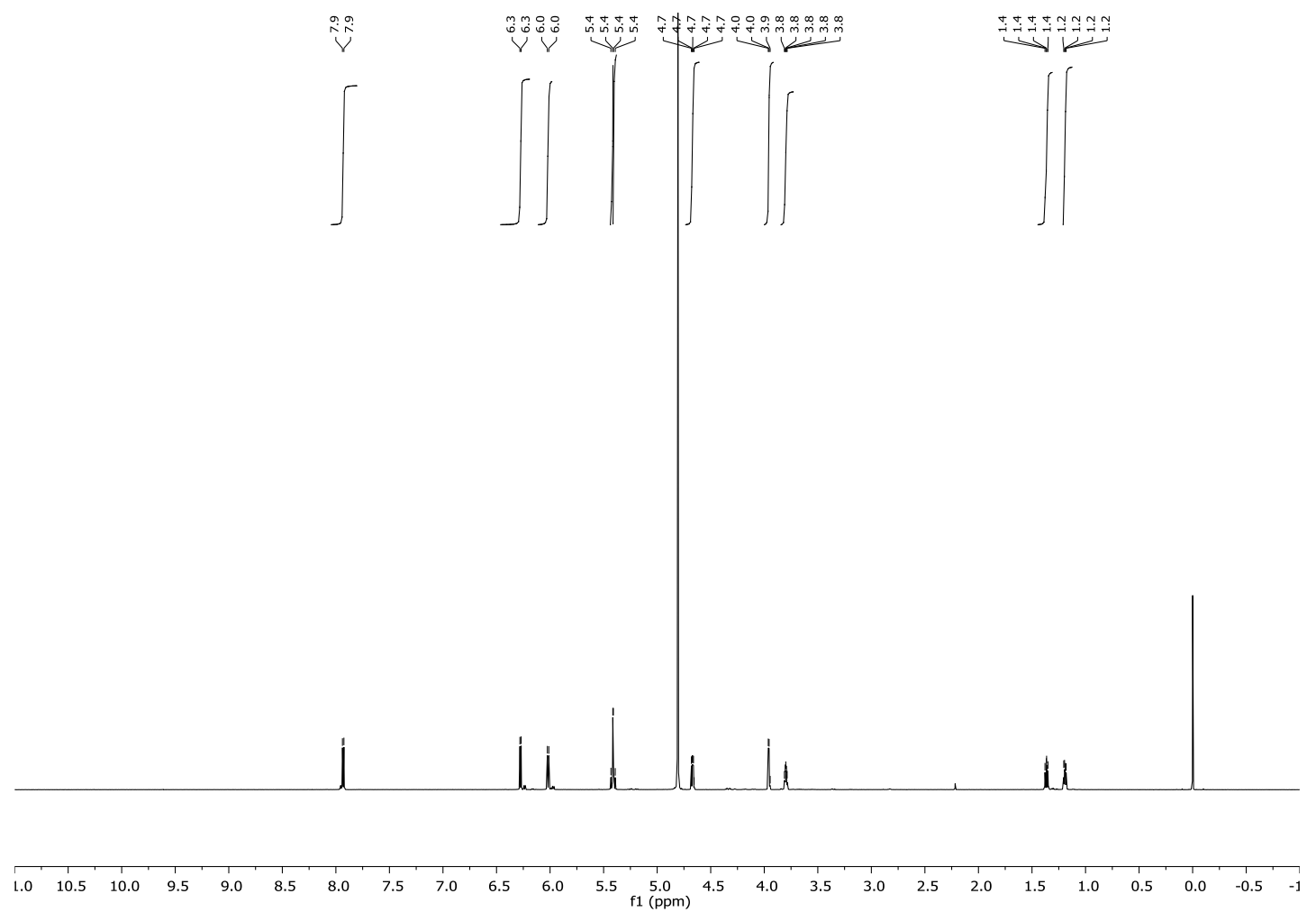

Figure S37. ${ }^{1} \mathrm{H}$ NMR (600 MHz, $\left.\mathrm{D}_{2} \mathrm{O}\right)$ spectrum of compound 15.
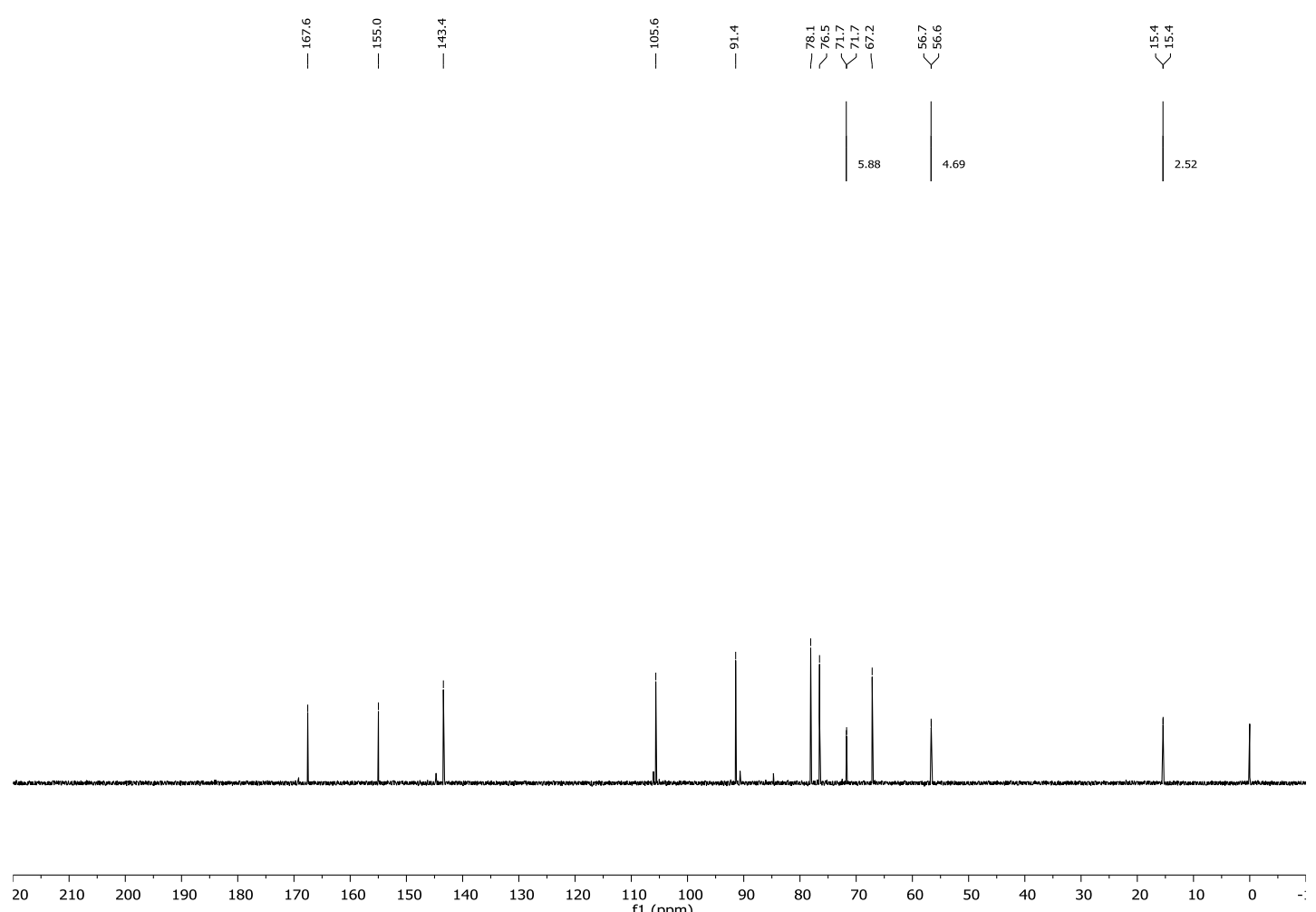

Figure S38. ${ }^{13} \mathrm{C}$ NMR (151 MHz, $\mathrm{D}_{2} \mathrm{O}$ ) spectrum of compound 15. 

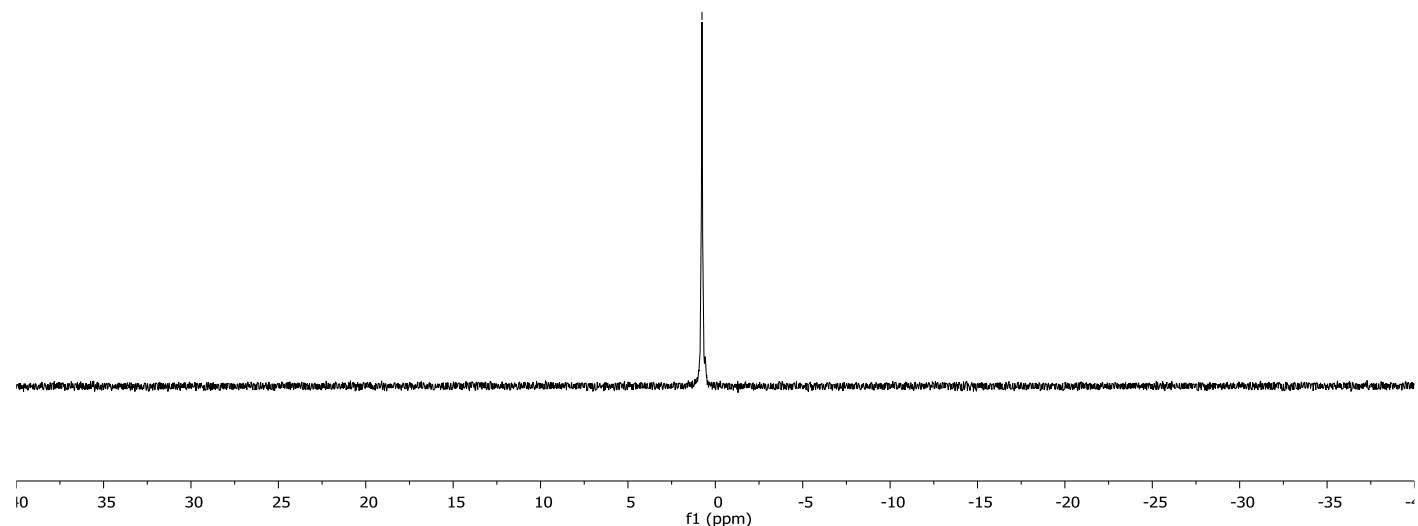

Figure S39. ${ }^{31} \mathrm{P}$ NMR (203 MHz, $\mathrm{D}_{2} \mathrm{O}$ ) spectrum of compound 15.

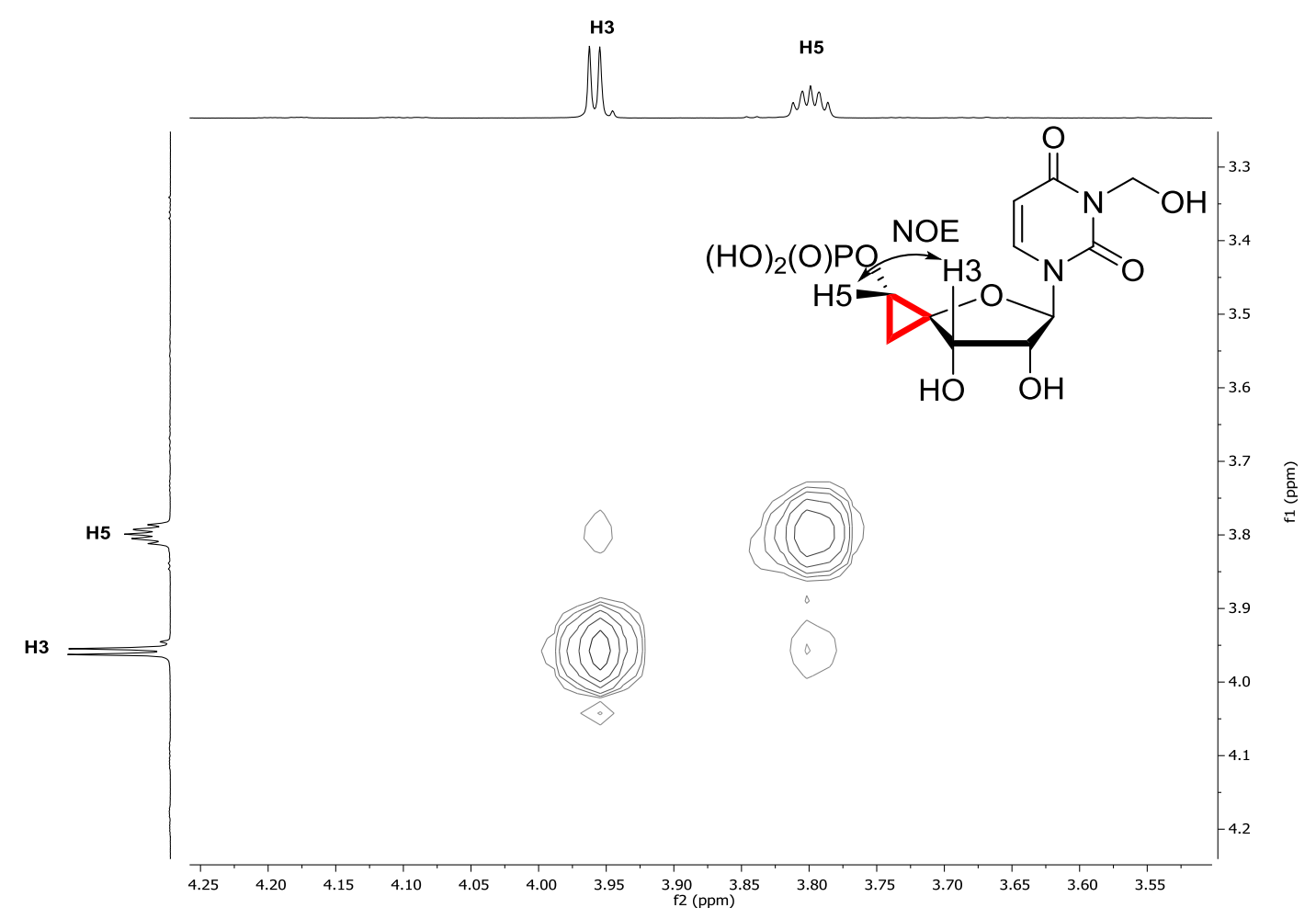

Figure S40. Partial NOESY $\left(600 \mathrm{MHz}, \mathrm{D}_{2} \mathrm{O}\right)$ of compound 15. 


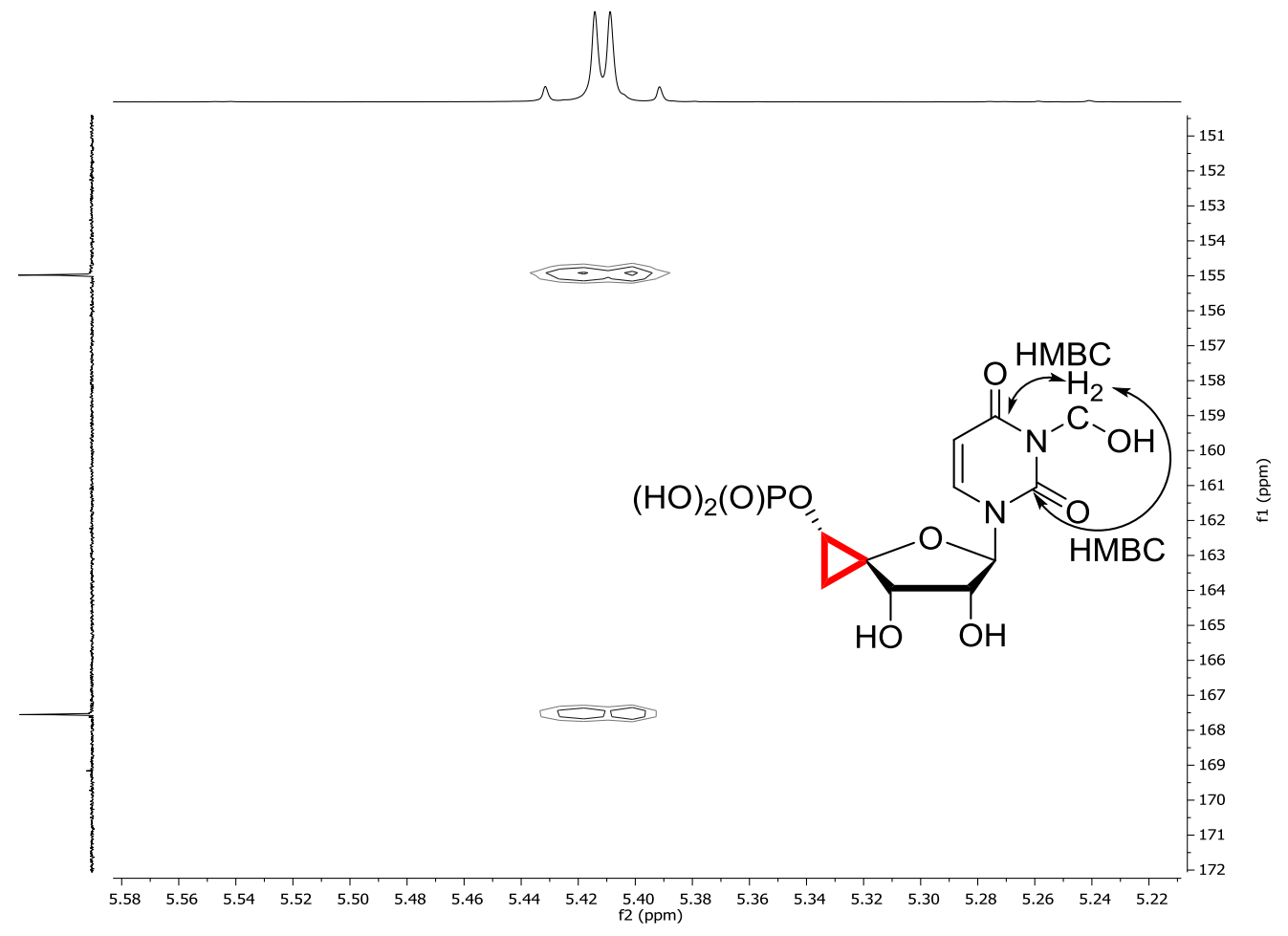

Figure S41. Partial HMBC $\left(600 \mathrm{MHz}, \mathrm{D}_{2} \mathrm{O}\right)$ of compound 15. 


\section{Compound 16}

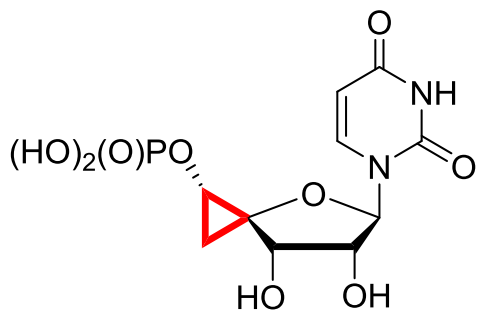

Compound $15(9.4 \mathrm{mg}, 25.7 \mu \mathrm{mol})$ was dissolved in $\mathrm{D}_{2} \mathrm{O}(0.60 \mathrm{~mL})$ and heated to $50{ }^{\circ} \mathrm{C}$ for $3 \mathrm{~d}$. The progress of reaction was monitored by ${ }^{1} \mathrm{H}$ NMR and finally the mixture evaporated to dryness. The remaining residue was dissolved in a few drops of methanol at $50{ }^{\circ} \mathrm{C}$ and compound 16 precipitated by the dropwise addition of $\mathrm{DCM}(2.00 \mathrm{~mL})$ at room temperature. The supernatant was removed by syringe and the precipitation protocol was repeated twice. Removal of methanediol and other derivatives of formaldehyde was ensured by ${ }^{1} \mathrm{H}$ NMR before compound 16 was dried by lyophilisation for $14 \mathrm{~h}$. The product was afforded as a colorless solid (8.6 mg, $25.6 \mu \mathrm{mol}$, quant.).

${ }^{1} \mathrm{H}$ NMR $\left(500 \mathrm{MHz}, \mathrm{D}_{2} \mathrm{O}\right) \delta(\mathrm{ppm}) 7.96(\mathrm{~d}, J=7.9 \mathrm{~Hz}, 1 \mathrm{H}), 6.24(\mathrm{~d}, J=6.9 \mathrm{~Hz}, 1 \mathrm{H}), 5.97(\mathrm{~d}, J=$ $7.8 \mathrm{~Hz}, 1 \mathrm{H}), 4.66(\mathrm{dd}, J=7.0,4.5 \mathrm{~Hz}, 1 \mathrm{H}), 3.95(\mathrm{~d}, J=4.5 \mathrm{~Hz}, 1 \mathrm{H}), 3.78(\mathrm{dt}, J=7.1,3.5 \mathrm{~Hz}, 1 \mathrm{H})$, $1.36(\mathrm{t}, J=8.1 \mathrm{~Hz}, 1 \mathrm{H}), 1.18(\mathrm{dd}, J=8.8,4.0 \mathrm{~Hz}, 1 \mathrm{H})$.

${ }^{13} \mathrm{C}$ NMR $\left(126 \mathrm{MHz}, \mathrm{D}_{2} \mathrm{O}\right) \delta(\mathrm{ppm})$ 169.2, 155.0, 144.7, 106.1, 90.6, 78.1, 76.6, 71.7 (d, J = 5.5 $\mathrm{Hz}), 56.6(\mathrm{~d}, J=3.1 \mathrm{~Hz}), 15.4$.

${ }^{31}$ P NMR (122 MHz, D 20$) \delta(p p m) 0.8$.

IR $(\mathrm{ATR}): \tilde{v}\left(\mathrm{~cm}^{-1}\right)=3234,1690,1467,1429,1381,1258,1203,1075$.

HR-MS (ESI): $m / z$ calcd for $\mathrm{C}_{10} \mathrm{H}_{12} \mathrm{~N}_{2} \mathrm{O}_{9}: 335.02859$; found: 335.02844 .

$[\alpha]_{\mathrm{D}}^{20}=36.3^{\circ}\left(\mathrm{c}=0.94, \mathrm{H}_{2} \mathrm{O}\right)$. 

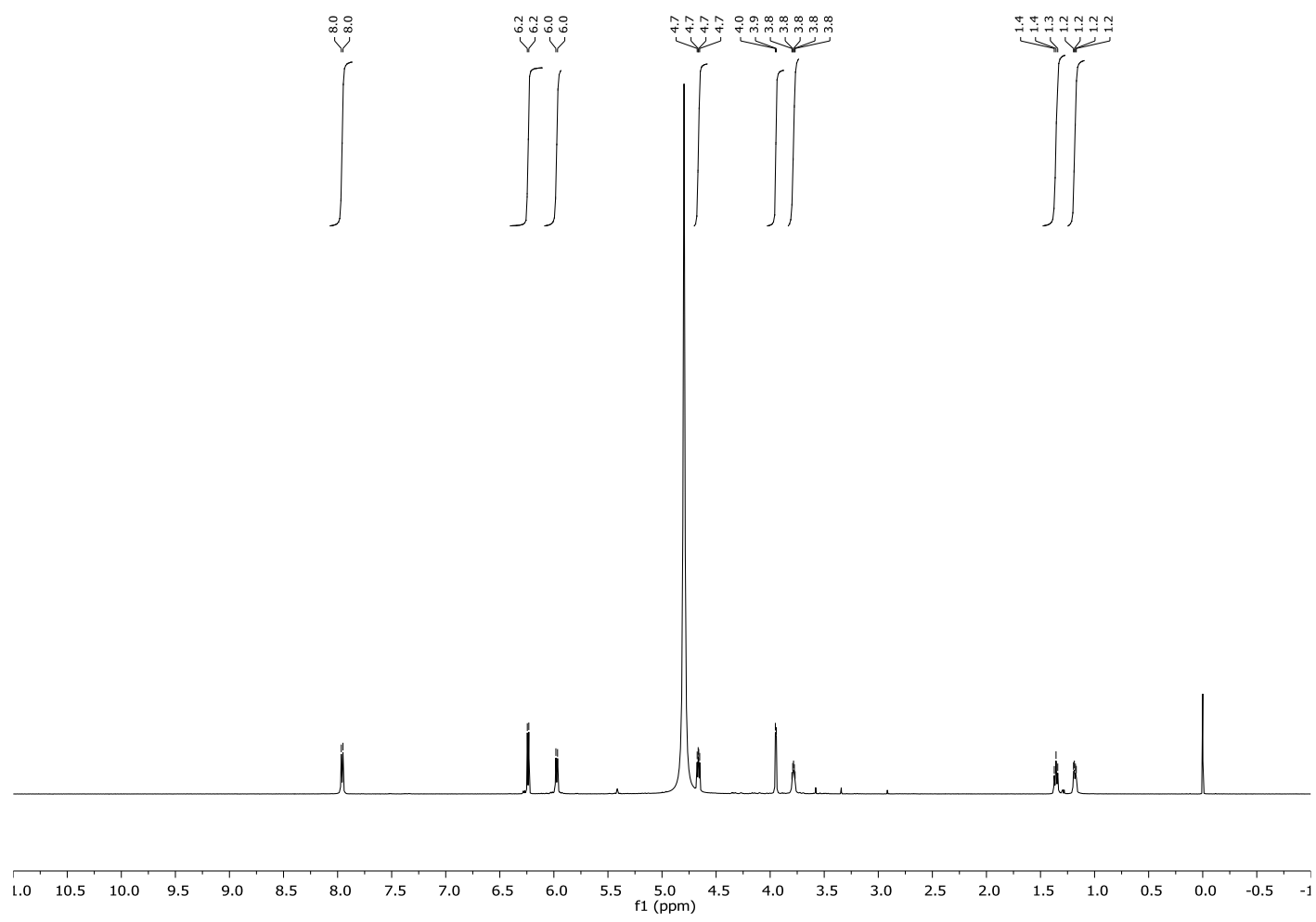

Figure S19. ${ }^{1} \mathrm{H}$ NMR (500 MHz, $\left.\mathrm{D}_{2} \mathrm{O}\right)$ of compound 16.
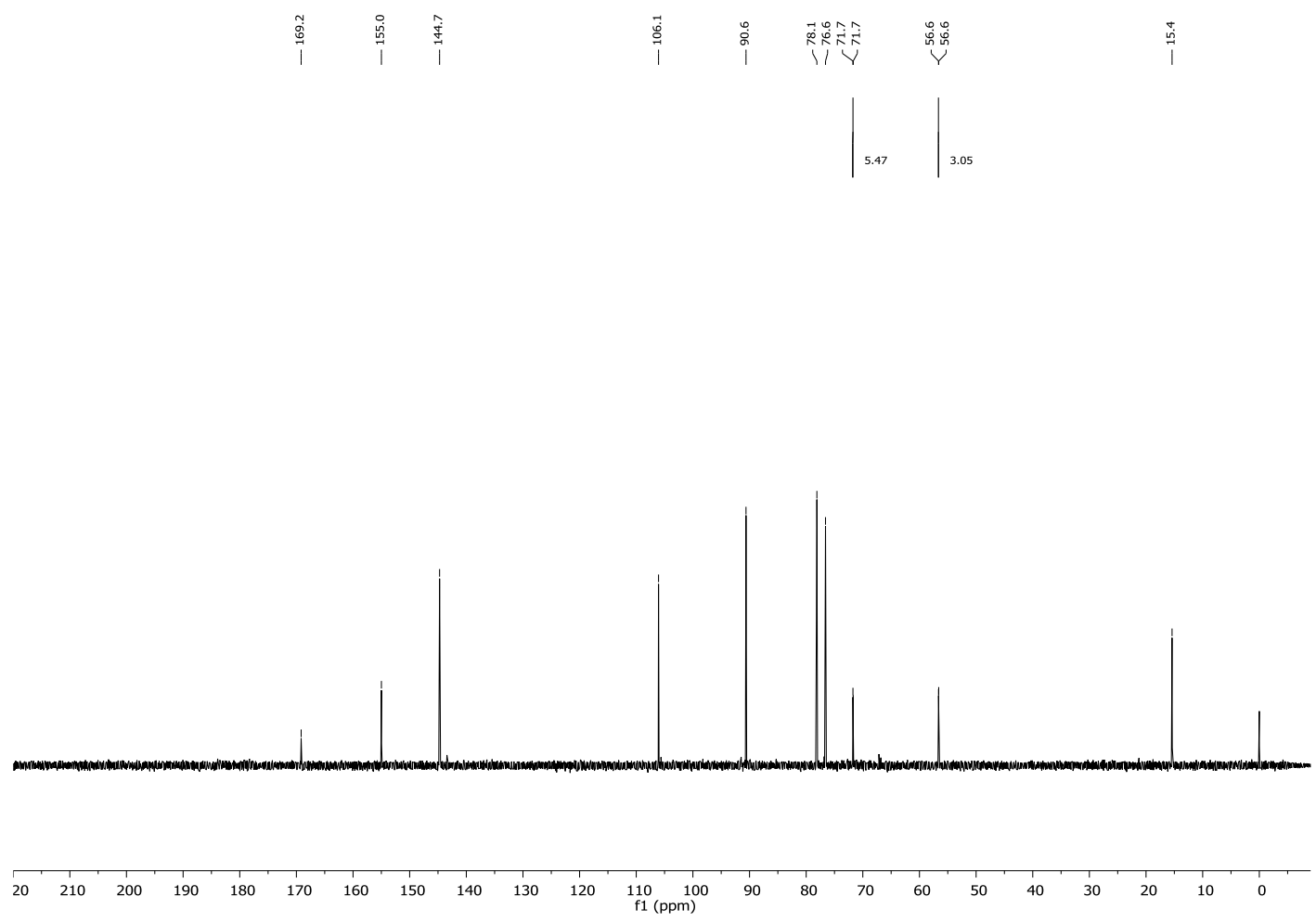

Figure S43. ${ }^{13} \mathrm{C}$ NMR (126 MHz, $\left.\mathrm{D}_{2} \mathrm{O}\right)$ of compound 16.

S45 


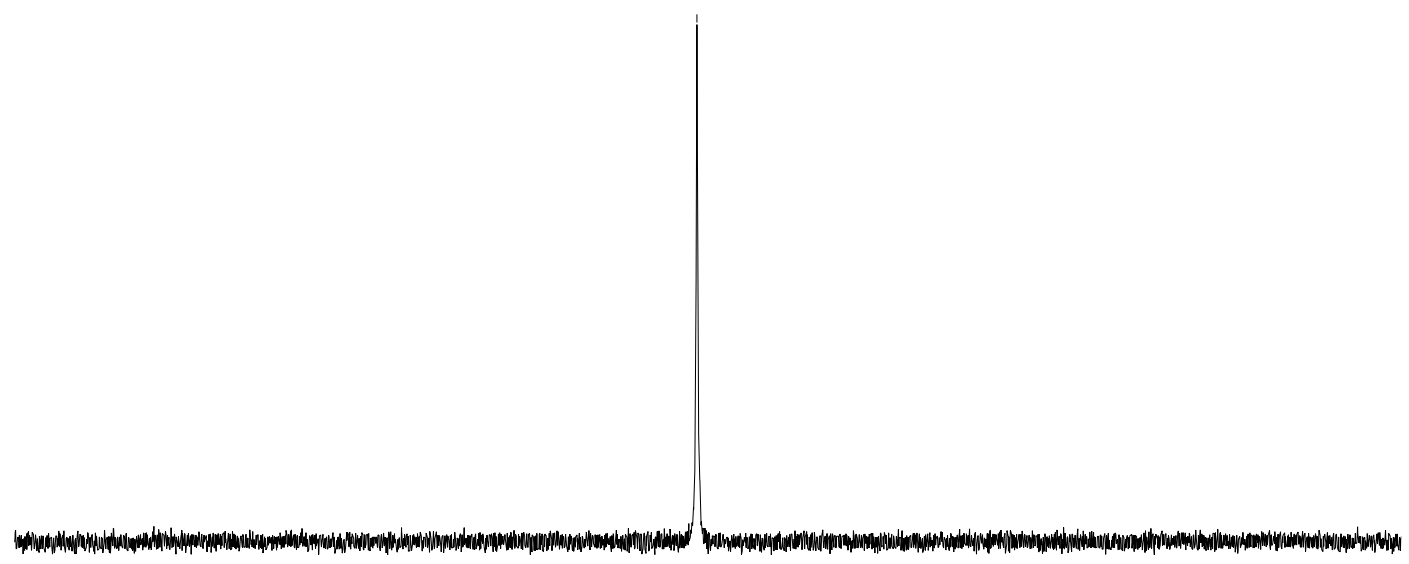

\begin{tabular}{llllllllllllllllllllllllll}
\hline 0 & 45 & 40 & 35 & 30 & 25 & 20 & 15 & 10 & 5 & $\underset{f 1}{0}(\mathrm{ppm})$ & -5 & -10 & -15 & -20 & -25 & -30 & -35 & -40 & -45 & -4
\end{tabular}

Figure S44. ${ }^{31} \mathrm{P}$ NMR (122 MHz, $\left.\mathrm{D}_{2} \mathrm{O}\right)$ of compound 16. 

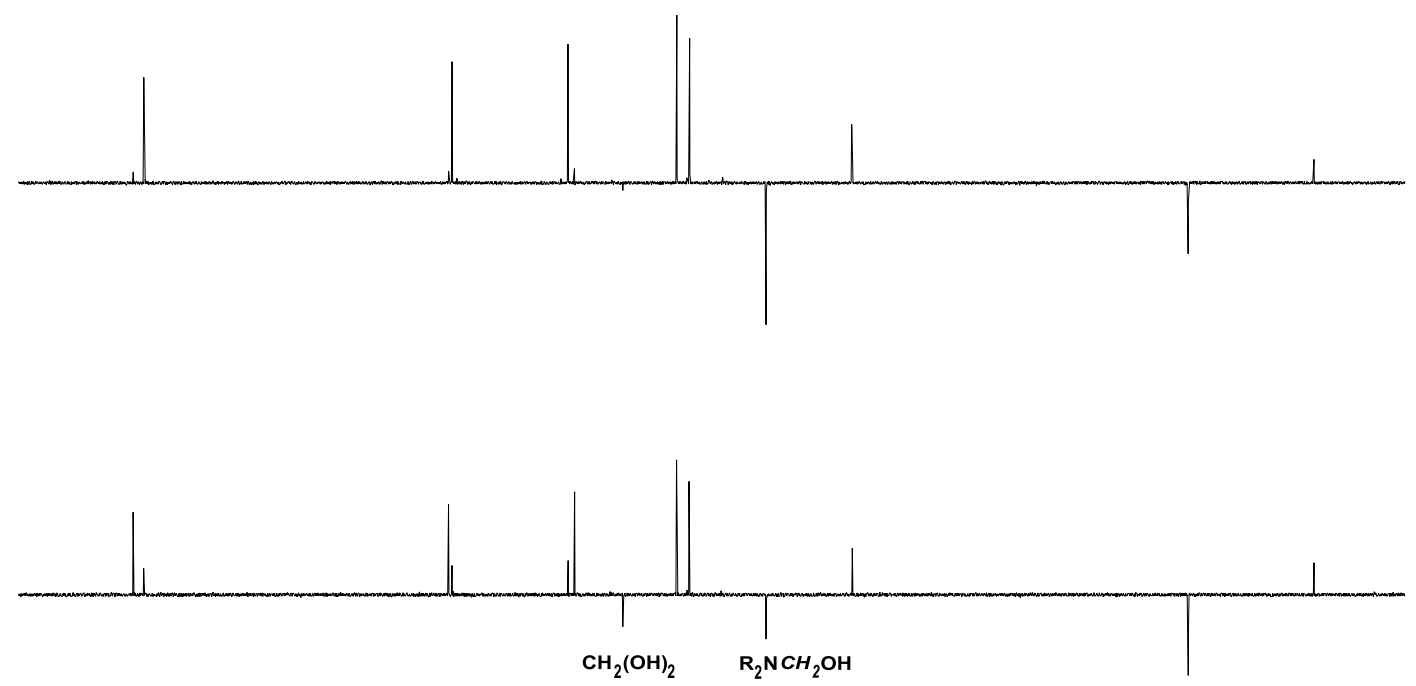

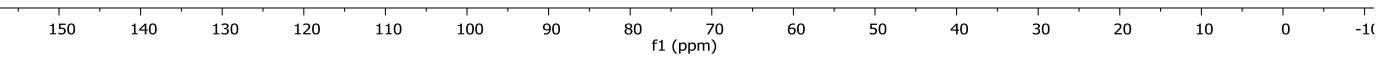

Figure S45. DEPT-135 monitored hydrolysis of hemiaminal 15 (top) under formation of $\mathbf{1 6}$ and formaldehyde monohydrate (bottom). 


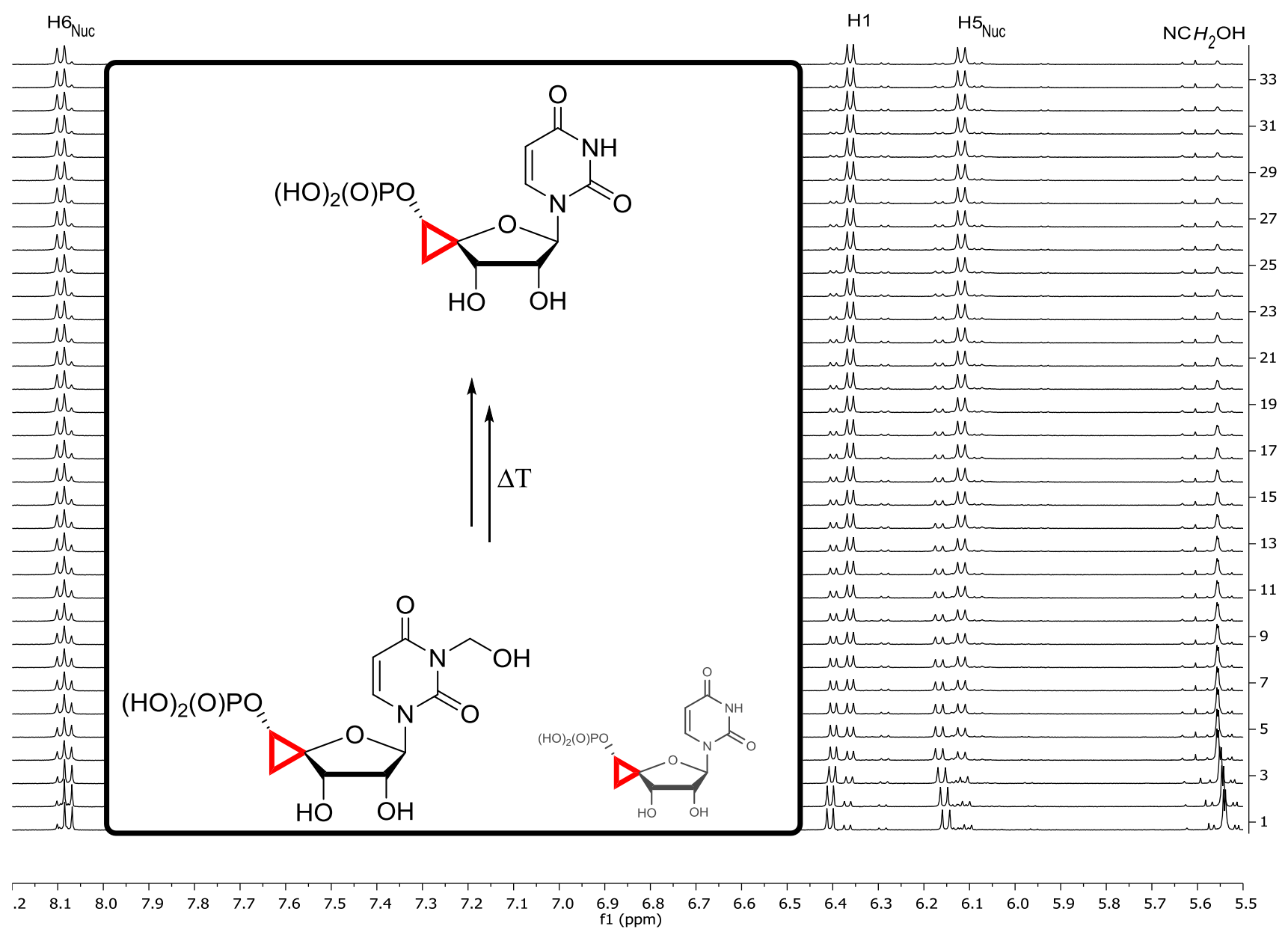

Figure 46. ${ }^{1} \mathrm{H}$ NMR analysis of temperature-promoted conversion of compound 15 to 16 by solvation in $\mathrm{D}_{2} \mathrm{O}$. Accuracy of temperature (set $50^{\circ} \mathrm{C}$ ) determined by measurement of ethylene glycol in DMSO- $d_{6}\left(45.7^{\circ} \mathrm{C}\right)$. 


\section{Compound 17}

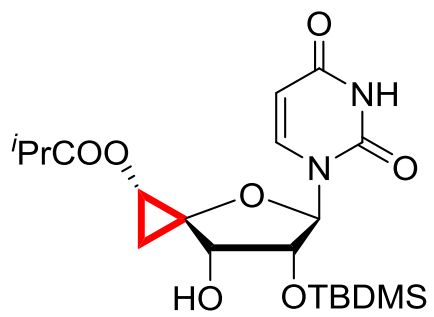

Compound 17 was prepared according to the procedure reported by Kreutz et al. ${ }^{[2]}$

Uridine derivative 6 (35.2 $\mathrm{mg}, 0.11 \mathrm{mmol}, 1.00$ eq.) was dissolved in terahydrofuran $(3.40 \mathrm{~mL})$ and pyridine ( $44.4 \mu \mathrm{L}, 0.55 \mathrm{mmol}, 5.00$ eq.). After the addition of TBDMSCl (36.2 mg, $0.24 \mathrm{mmol}$, 2.20 eq.) and $\mathrm{AgNO}_{3}(40.8 \mathrm{mg}, 0.24 \mathrm{mmol}, 2.20$ eq.) the flask was covered with foil and the suspension stirred for $16 \mathrm{~h}$ in the dark. The reaction was terminated by filtration and the plug was rinsed with dichloromethane $(3 x, 5.00 \mathrm{~mL})$. The organic phase was washed with water $(10.0 \mathrm{~mL})$, dried over $\mathrm{Na}_{2} \mathrm{SO}_{4}$, filtered and evaporated to dryness. Column purification $\left(\mathrm{SiO}_{2}\right.$, pentane/EtOAc, $5: 1$ to $1: 1)$ afforded the desired silylated compound 17 as a colorless solid $(35.7 \mathrm{mg}, 0.81 \mathrm{mmol}$, $81 \%$ yield).

${ }^{1} \mathrm{H}$ NMR $\left(300 \mathrm{MHz}_{\mathrm{CDCl}}\right) \delta(\mathrm{ppm}) 8.15(\mathrm{~s}, 1 \mathrm{H}), 7.38(\mathrm{~d}, J=8.2 \mathrm{~Hz}, 1 \mathrm{H}), 6.22(\mathrm{dt}, J=6.6,0.5$ $\mathrm{Hz}, 1 \mathrm{H}$ ), 5.76 (ddd, $J=8.2,2.4,0.5 \mathrm{~Hz}, 1 \mathrm{H}$ ), 4.42 (dd, $J=6.5,4.9 \mathrm{~Hz}, 1 \mathrm{H}$ ), 3.90 (dd, J = 4.9, 3.0 $\mathrm{Hz}, 1 \mathrm{H}$ ), $3.81(\mathrm{dd}, J=7.9,4.7 \mathrm{~Hz}, 1 \mathrm{H}), 2.71(\mathrm{~d}, J=3.1 \mathrm{~Hz}, 1 \mathrm{H}), 2.59$ (hept, $J=7.0 \mathrm{~Hz}, 1 \mathrm{H}), 1.45$ (dd, $J=8.8,8.0 \mathrm{~Hz}, 1 \mathrm{H}), 1.25-1.14(\mathrm{~m}, 1 \mathrm{H}), 1.17(\mathrm{~d}, J=7.0 \mathrm{~Hz}, 3 \mathrm{H}), 1.13(\mathrm{~d}, J=7.0 \mathrm{~Hz}, 3 \mathrm{H})$, $0.90(\mathrm{~s}, 9 \mathrm{H}), 0.10(\mathrm{~s}, 3 \mathrm{H}), 0.07(\mathrm{~s}, 3 \mathrm{H})$.

${ }^{13} \mathrm{C}$ NMR (126 MHz, $\left.\mathrm{CDCl}_{3}\right) \delta$ (ppm) 179.2, 162.2, 150.1, 139.7, 103.1, 86.8, 75.3, 72.6, 67.8, 53.0, 33.6, 25.5, 18.9, 18.8, 17.9, 12.1, -4.9, -5.1.

IR (ATR): $\tilde{v}\left(\mathrm{~cm}^{-1}\right)=3451,3219,3069,2933,2859,1694,1465,1383,1257,1194,1154,1110$ 1022.

HR-MS (ESI): $m / z$ calcd for $\mathrm{C}_{20} \mathrm{H}_{32} \mathrm{~N}_{2} \mathrm{O}_{7} \mathrm{SiNa}^{+}:$463.18710; found: 463.18752 .

$[\alpha]_{\mathrm{D}}^{20}=-13.7^{\circ}\left(\mathrm{c}=0.25, \mathrm{CHCl}_{3}\right)$. 

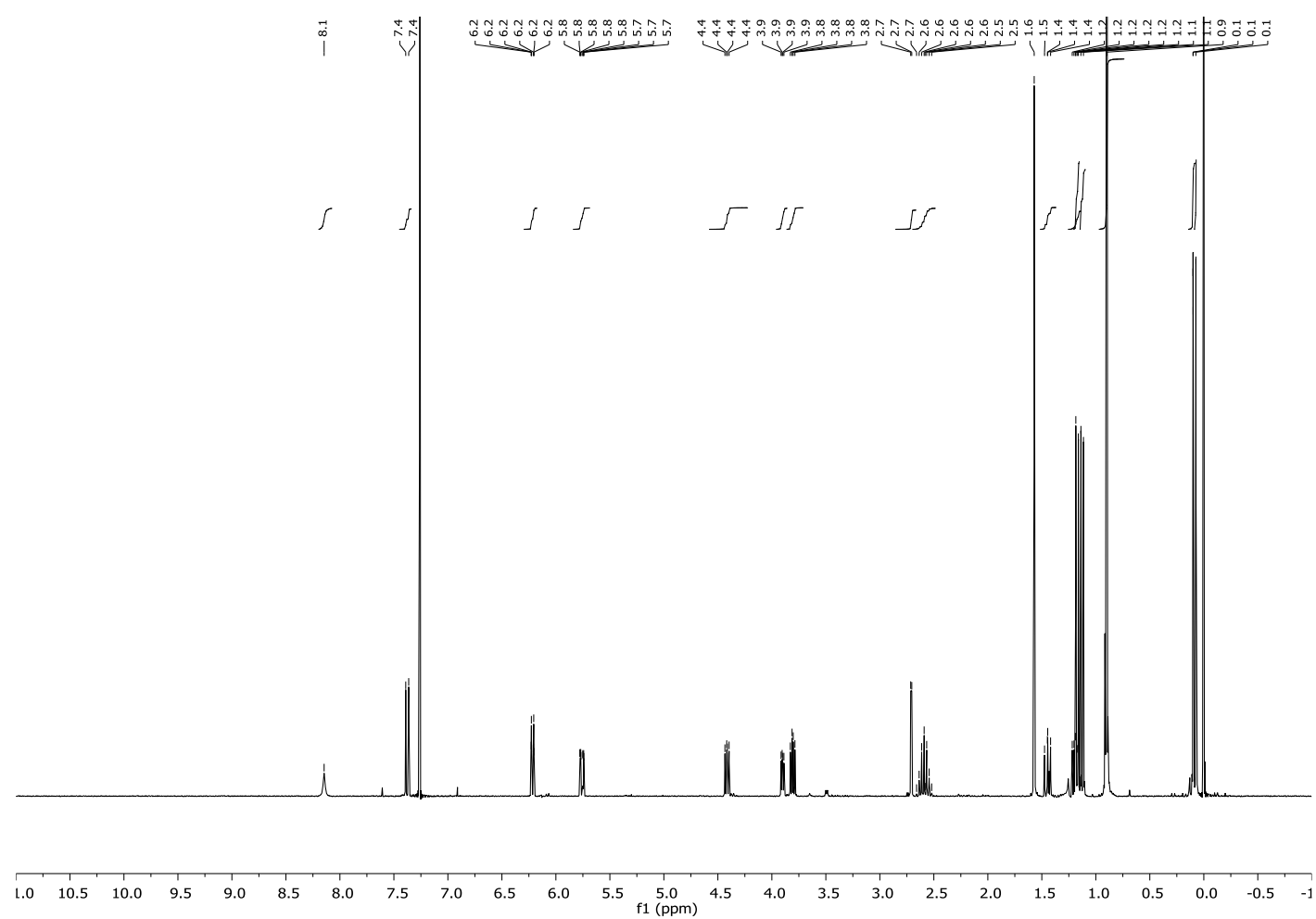

Figure S47. ${ }^{1} \mathrm{H} \mathrm{NMR}\left(300 \mathrm{MHz}, \mathrm{CDCl}_{3}\right)$ of compound 17.

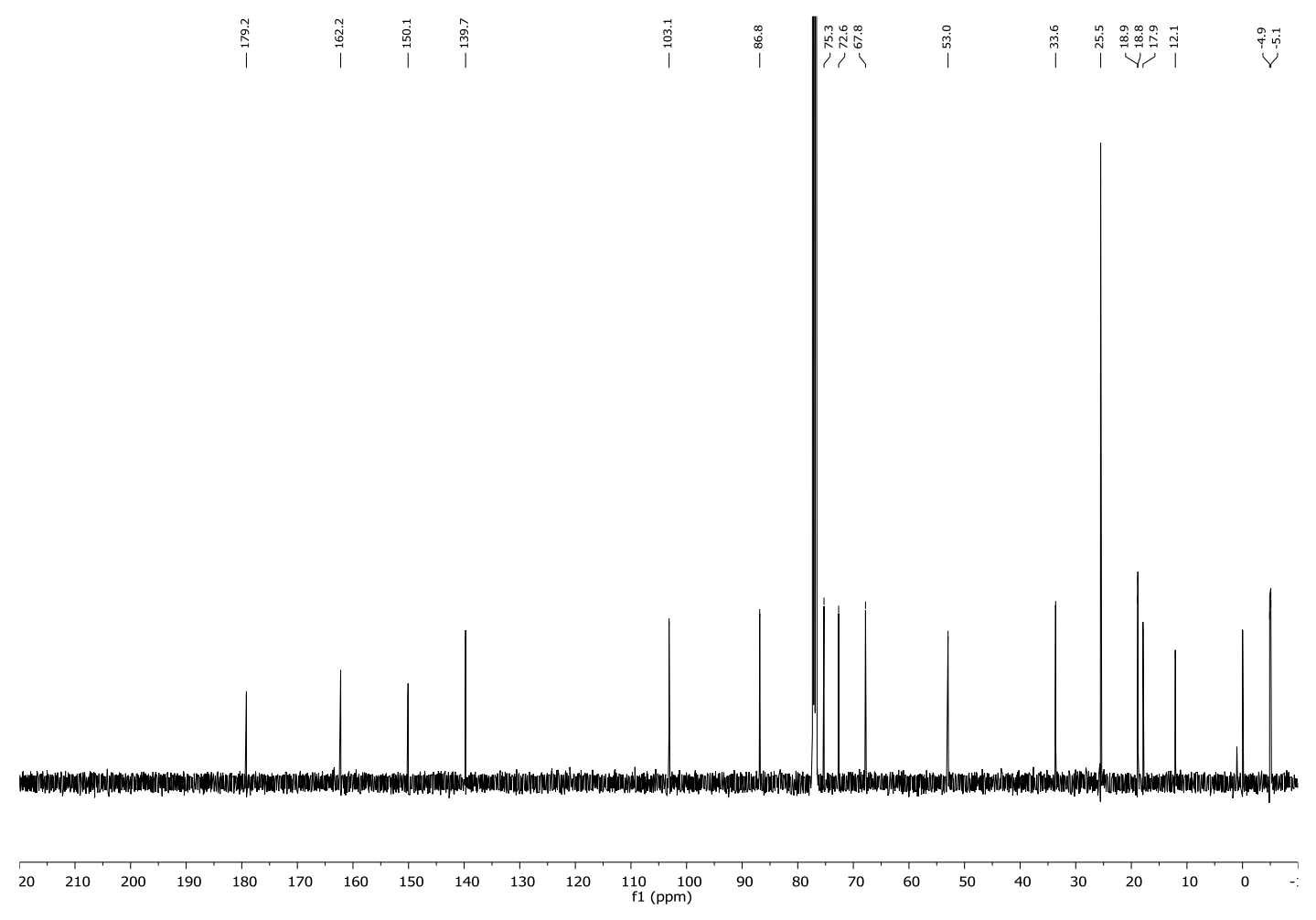

Figure S48. ${ }^{13} \mathrm{C}$ NMR (126 MHz, $\mathrm{CDCl}_{3}$ ) of compound 17. 


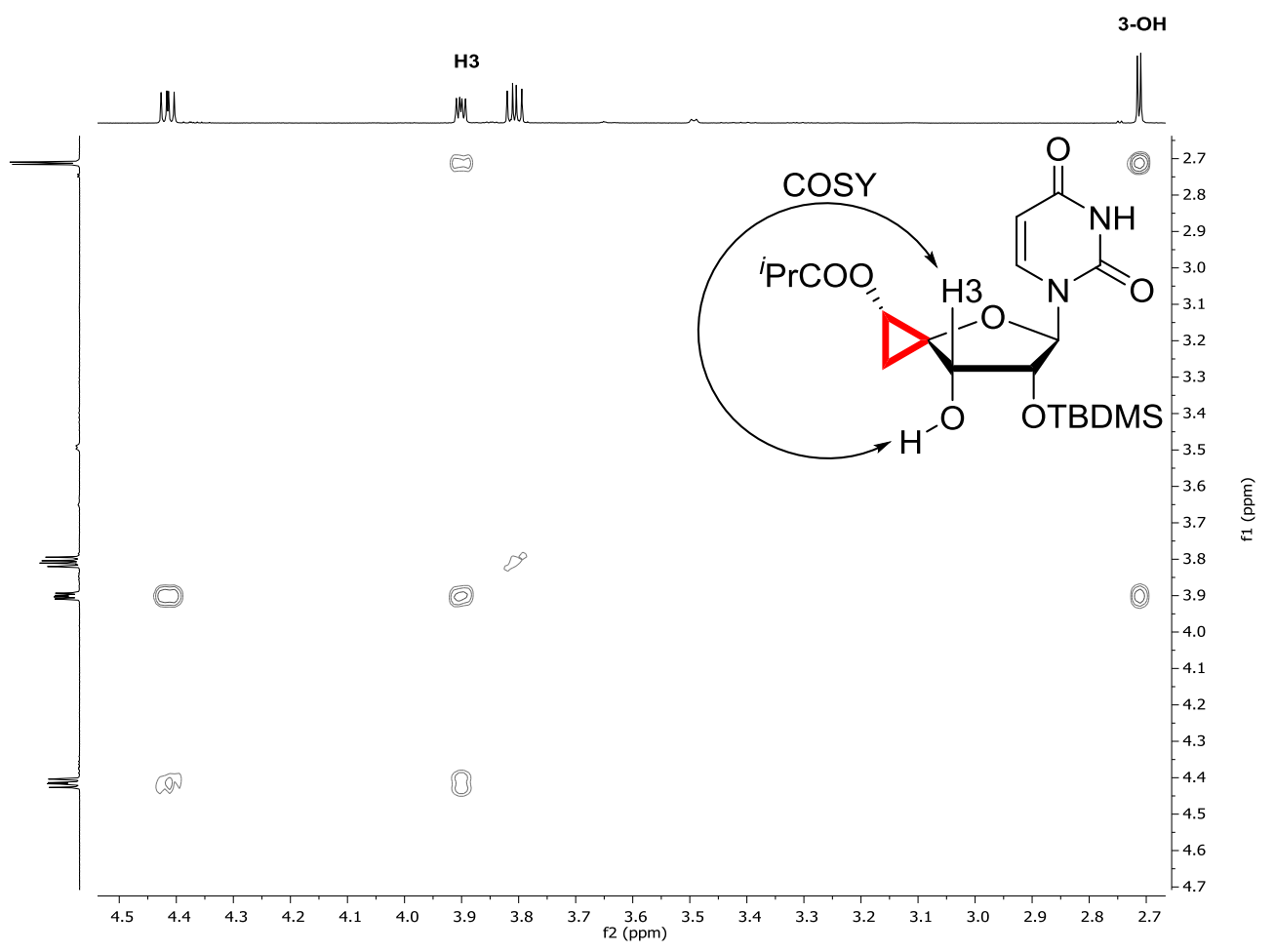

Figure S49. Partial COSY ( $\left.300 \mathrm{MHz}, \mathrm{CDCl}_{3}\right)$ of compound 17. 


\section{Compound 18}

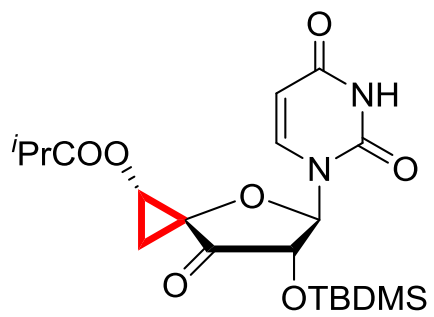

Before use, uridine derivative 17 was azeotropically distilled with toluene $(3 x, 1.00 \mathrm{~mL})$ and dried for $0.5 \mathrm{~h}$ at high vacuum. Compound 17 (36.0 mg, $81.7 \mu \mathrm{mol}, 1.00 \mathrm{eq}$.) was dissolved in DCM $(2.00 \mathrm{~mL})$ and after the addition of DMP $(67.9 \mathrm{mg}, 0.16 \mathrm{mmol}, 2.00 \mathrm{eq}$.) the mixture was stirred for $3 \mathrm{~h}$ at room temperature. The reaction was terminated by the addition of diethyl ether $(20.0 \mathrm{~mL})$ and a 1:1 mixture of saturated aqueous solutions of $\mathrm{Na}_{2} \mathrm{~S}_{2} \mathrm{O}_{3}$ and $\mathrm{NaHCO}_{3}(10.0 \mathrm{~mL})$, cooled to $0{ }^{\circ} \mathrm{C}$ before use. The mixture was shaken for $5 \mathrm{~min}$ and the organic phase was washed with brine $(10.0 \mathrm{~mL})$. The organic layer was finally dried over $\mathrm{Na}_{2} \mathrm{SO}_{4}$, filtered and evaporated to dryness. Purification by column chromatography $\left(\mathrm{SiO}_{2}\right.$, pentane/EtOAc, 5:1 to 1:1) afforded the 3-oxo nucleoside 18 (18.0 mg, $41.0 \mu \mathrm{mol}, 50 \%$ yield) as a colorless foam. For further experiments column purification was omitted.

${ }^{1} \mathrm{H}$ NMR $\left(600 \mathrm{MHz}, \mathrm{CDCl}_{3}\right) \delta(\mathrm{ppm}) 8.27(\mathrm{~s}, 1 \mathrm{H}), 7.40(\mathrm{~d}, J=8.2 \mathrm{~Hz}, 1 \mathrm{H}), 6.40(\mathrm{~d}, J=8.2 \mathrm{~Hz}, 1 \mathrm{H})$, $5.84(\mathrm{dd}, J=8.2,2.3 \mathrm{~Hz}, 1 \mathrm{H}), 4.56$ (d, $J=8.2 \mathrm{~Hz}, 1 \mathrm{H}), 3.99$ (dd, $J=7.7,5.4 \mathrm{~Hz}, 1 \mathrm{H}), 2.61$ (p, $J=$ $6.9 \mathrm{~Hz}, 1 \mathrm{H}), 1.91$ (t, J=7.8 Hz, 1H), 1.60 (dd, J=7.9, $5.4 \mathrm{~Hz}, 1 \mathrm{H}), 1.18(\mathrm{~d}, J=7.0 \mathrm{~Hz}, 3 \mathrm{H}), 1.13$ (d, $J=7.0 \mathrm{~Hz}, 3 \mathrm{H}), 0.87$ (d, $J=0.8 \mathrm{~Hz}, 9 \mathrm{H}), 0.13(\mathrm{~s}, 3 \mathrm{H}), 0.04(\mathrm{~s}, 3 \mathrm{H})$.

${ }^{13} \mathrm{C}$ NMR $\left(151 \mathrm{MHz}, \mathrm{CDCl}_{3}\right) \delta(\mathrm{ppm}) 205.8,178.3,161.9,149.9,139.0,104.0,86.0,75.5,65.8$, $56.2,33.5,25.4,19.1,18.9,18.8,18.1,-4.6,-5.3$.

IR (ATR): $\tilde{v}\left(\mathrm{~cm}^{-1}\right)=3211,2957,2932,2891,2858,1766,1695,1461,1382,1259,1161,1068$ 1015.

HR-MS (ESI): $m / z$ calcd for $\mathrm{C}_{20} \mathrm{H}_{30} \mathrm{~N}_{2} \mathrm{O}_{7} \mathrm{SiNa}^{+}:$461.17145; found: 461.17211.

$[\alpha]_{\mathrm{D}}^{20}=17.4^{\circ}\left(\mathrm{c}=0.29, \mathrm{CHCl}_{3}\right)$. 

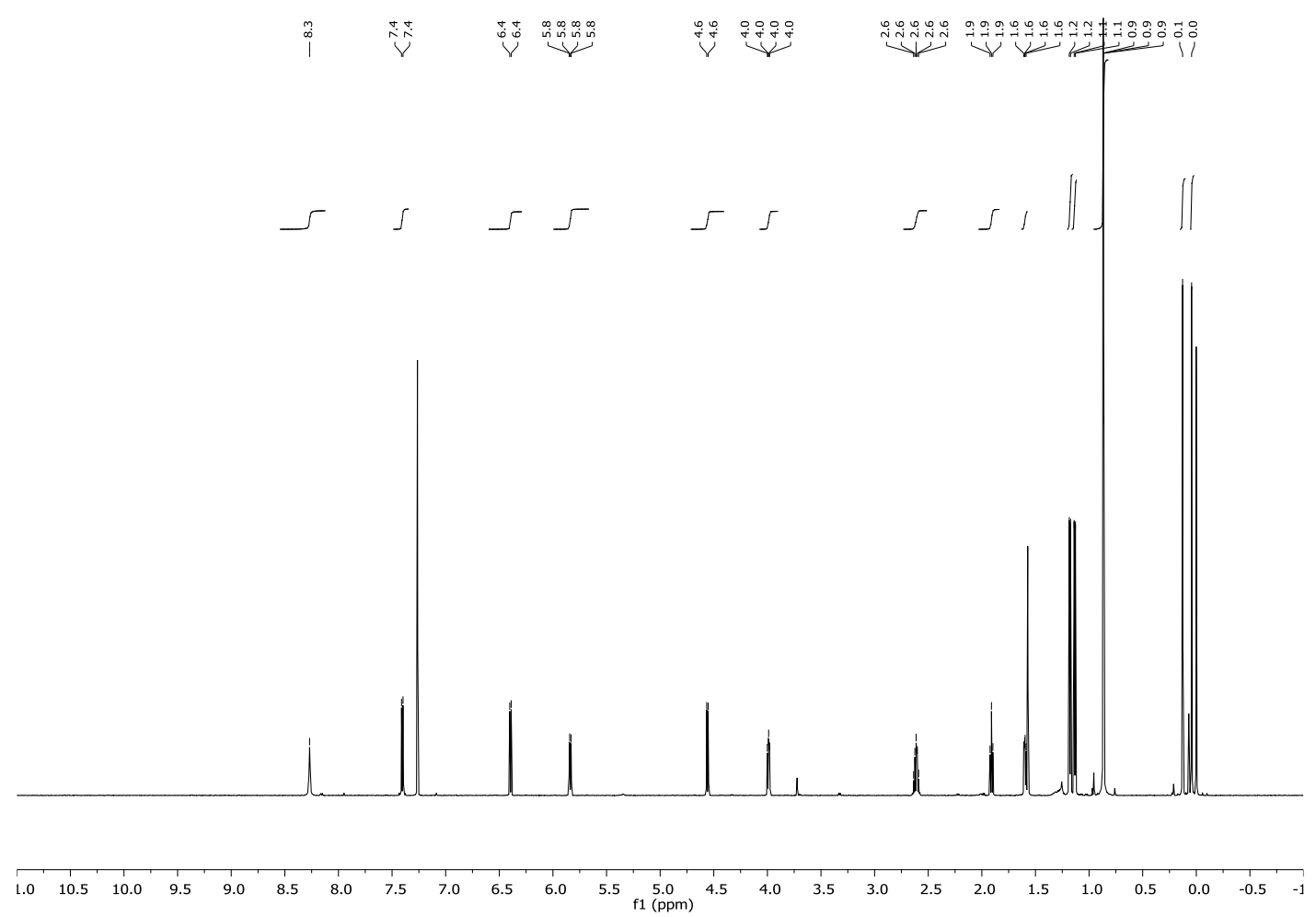

Figure S50. ${ }^{1} \mathrm{H} \mathrm{NMR}\left(600 \mathrm{MHz}, \mathrm{CDCl}_{3}\right)$ of compound 18.

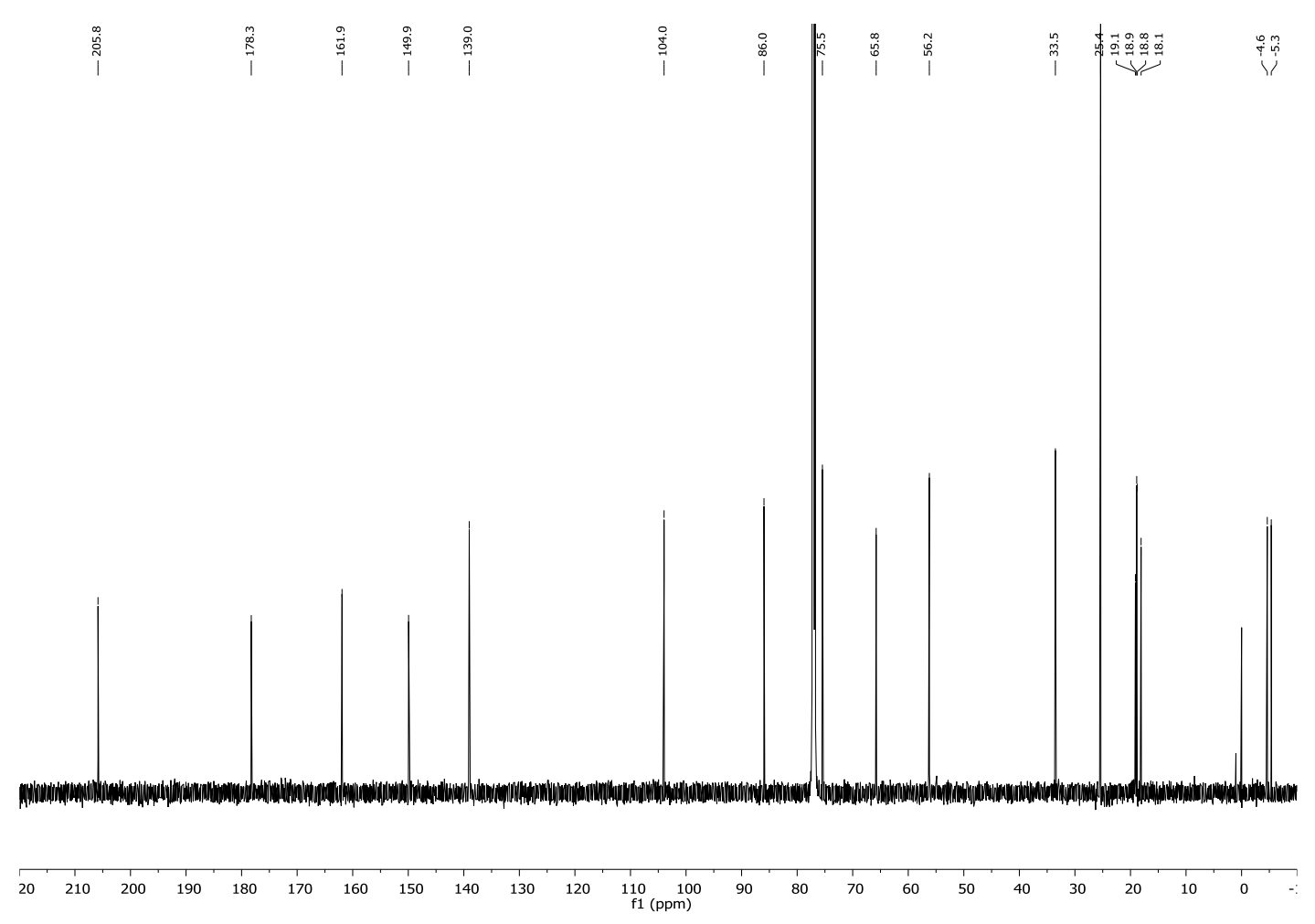

Figure S51. ${ }^{13} \mathrm{C}$ NMR (151 MHz, $\left.\mathrm{CDCl}_{3}\right)$ of compound 18. 


\section{Compound 19}

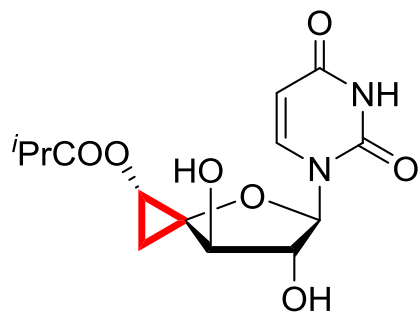

Compound 18 (18.0 mg, $41.0 \mu \mathrm{mol}, 1.00$ eq.) was dissolved in $\mathrm{MeOH}$ (1.00 mL) and cooled to $0{ }^{\circ} \mathrm{C} . \mathrm{NaBH}_{4}(1.7 \mathrm{mg}, 45.1 \mu \mathrm{mol}, 1.10$ eq.) was added and the reaction mixture, stirred for $0.5 \mathrm{~h}$ and finally evaporated to dryness. EtOAc $(10.0 \mathrm{~mL})$ was added to the salty residue and the mixture was partitioned against saturated $\mathrm{NaCl}(5.00 \mathrm{~mL})$. The aqueous layer was extracted with EtOAc $(3 x, 5.00 \mathrm{~mL})$, the combined organic phases were dried over $\mathrm{Na}_{2} \mathrm{SO}_{4}$, filtered and evaporated to dryness. The oily residue was submitted to desilylation without further purification by dissolving in THF (1.00 mL) and addition of TBAF. $3 \mathrm{H}_{2} \mathrm{O}\left(14.2 \mathrm{mg}, 45.1 \mu \mathrm{mol}, 1.10 \mathrm{eq}\right.$.) at $0{ }^{\circ} \mathrm{C}$. The reaction mixture was stirred for $3 \mathrm{~h}$ and finally diluted with EtOAc $(10.0 \mathrm{~mL})$. A saturated aqueous solution of $\mathrm{NaHCO}_{3}(5.00 \mathrm{~mL})$ was added and the aqueous layer was extracted with EtOAc $(3 x, 5.00 \mathrm{~mL})$. The combined organic phases were dried over $\mathrm{Na}_{2} \mathrm{SO}_{4}$, filtered and evaporated to dryness. Column purification $\left(\mathrm{SiO}_{2}, \mathrm{DCM} / \mathrm{MeOH}, 20: 1\right.$ to 10:1) afforded a diastereomeric mixture of the desired D-xylose configured nucleoside 19 and the D-ribose configured carbohydrate scaffold 6 as a colorless oil (dr 5.9:1). Purification by HPLC (C18, isocratic, $\left.\mathrm{CH}_{3} \mathrm{CN}: \mathrm{H}_{2} \mathrm{O}, 70: 30, t_{\mathrm{R}}=2.67 \mathrm{~min}\right)$ afforded compound $19(8.9 \mathrm{mg}, 27.3 \mathrm{mmol}, 67 \%$ yield).

${ }^{1} \mathrm{H}$ NMR $\left(300 \mathrm{MHz}, \mathrm{CDCl}_{3}\right) \delta(\mathrm{ppm}) 9.61(\mathrm{~s}, 1 \mathrm{H}), 7.55(\mathrm{~d}, J=8.1 \mathrm{~Hz}, 1 \mathrm{H}), 5.98(\mathrm{~d}, J=0.9 \mathrm{~Hz}, 1 \mathrm{H})$, $5.70(\mathrm{~d}, J=8.3 \mathrm{~Hz}, 1 \mathrm{H}), 4.43(\mathrm{~s}, 1 \mathrm{H}), 4.03$ (t, $J=6.6 \mathrm{~Hz}, 1 \mathrm{H}), 3.98(\mathrm{~d}, J=1.4 \mathrm{~Hz}, 1 \mathrm{H}$ ), 2.63 (hept, $J=6.9 \mathrm{~Hz}, 1 \mathrm{H}), 1.41(\mathrm{~d}, J=6.6 \mathrm{~Hz}, 2 \mathrm{H}), 1.19(\mathrm{~d}, J=7.0 \mathrm{~Hz}, 3 \mathrm{H}), 1.15(\mathrm{~d}, J=7.0 \mathrm{~Hz}, 3 \mathrm{H})$.

${ }^{13} \mathrm{C}$ NMR (126 MHz, $\left.\mathrm{CDCl}_{3}\right) \delta(\mathrm{ppm})$ 179.7, 163.5, 150.9, 140.6, 101.9, 92.2, 81.5, 77.6, 70.1, 49.8, 33.7, 18.9, 18.9, 16.7.

IR (ATR): $\tilde{v}\left(\mathrm{~cm}^{-1}\right)=3388,2958,2925,2856,1686,1466,1387,1262,1193,1158,1108,1059$, 1017.

HR-MS (ESI): $m / z$ calcd for $\mathrm{C}_{14} \mathrm{H}_{18} \mathrm{~N}_{2} \mathrm{O}_{7} \mathrm{Na}^{+}: 349.10062$; found: 349.10084 .

$[\alpha]_{\mathrm{D}}^{20}=-47.2^{\circ}\left(\mathrm{c}=0.18, \mathrm{CHCl}_{3}\right)$. 

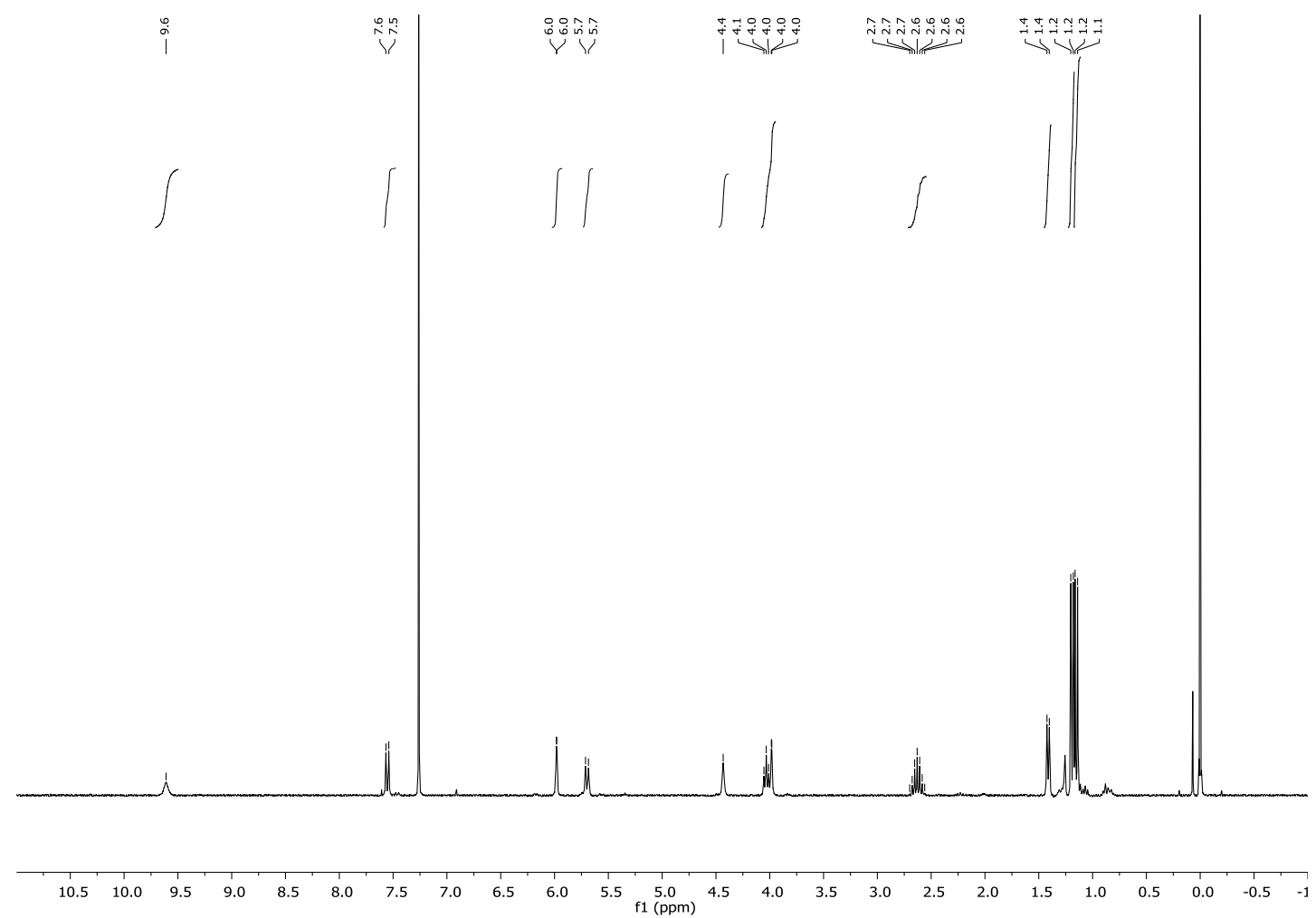

Figure S52. ${ }^{1} \mathrm{H} \mathrm{NMR}\left(300 \mathrm{MHz}, \mathrm{CDCl}_{3}\right)$ of compound 19.
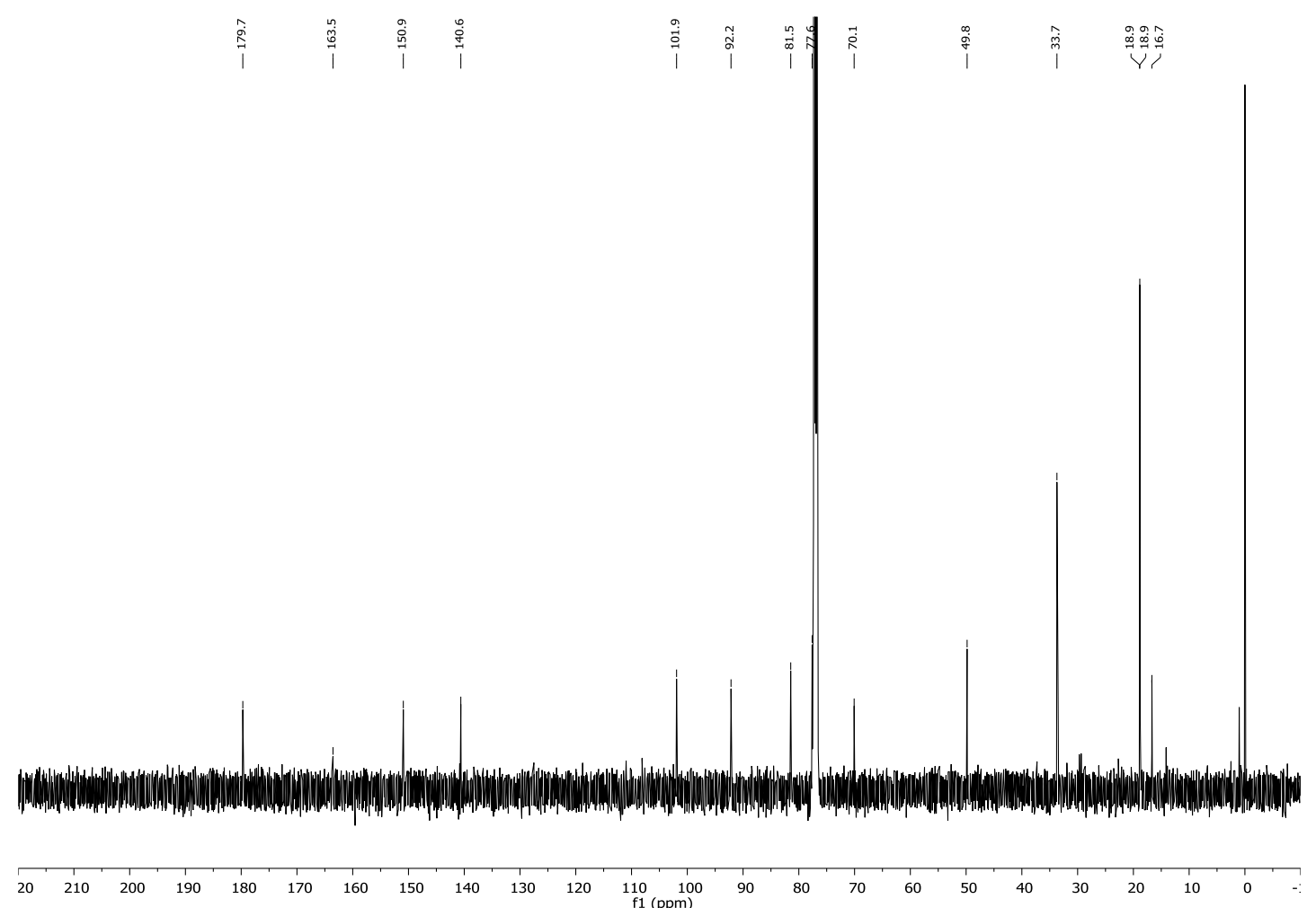

Figure S53. ${ }^{13} \mathrm{C} \mathrm{NMR}\left(126 \mathrm{MHz}, \mathrm{CDCl}_{3}\right)$ of compound 19. 


\section{Compound 20}

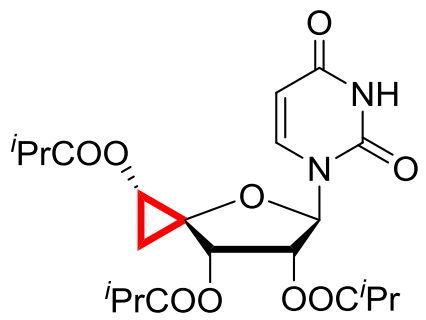

After uridine derivative 6 (48.4 mg, $148 \mu \mathrm{mol}, 1.00$ eq.) was dissolved in pyridine $(1.00 \mathrm{~mL})$, isobutyric anhydride (0.73 mL, $4.38 \mathrm{mmol}, 30.0$ eq.) and DMAP (1.80 mg, $14.7 \mu \mathrm{mol}, 0.10$ eq.) were added. The reaction mixture was stirred for $16 \mathrm{~h}$ at room temperature and finally DCM $(10.0 \mathrm{~mL})$ and a saturated aqueous solution of $\mathrm{NaHCO}_{3}(5.00 \mathrm{~mL})$ were added. The aqueous phase was extracted with DCM $(3 \mathrm{x}, 5.00 \mathrm{~mL})$, the combined organic phases were dried over $\mathrm{Na}_{2} \mathrm{SO}_{4}$, filtered and evaporated to dryness. Purification by column chromatography $\left(\mathrm{SiO}_{2}\right.$, pentane/ethyl acetate, $3: 1$ to 1:1) afforded the esterificated uridine derivative 20 (68.8 mg, $147 \mu \mathrm{mol}, 98 \%$ yield) as a colorless foam.

For biological investigations compound $\mathbf{2 0}$ was previously purified by HPLC (C18, isocratic, $\left.\mathrm{CH}_{3} \mathrm{CN}: \mathrm{H}_{2} \mathrm{O}, 70: 30, t_{\mathrm{R}}=5.64 \mathrm{~min}\right)$.

${ }^{1} \mathrm{H}$ NMR $\left(500 \mathrm{MHz}, \mathrm{CHCl}_{3}\right) \delta(\mathrm{ppm}) 8.21(\mathrm{~s}, 1 \mathrm{H}), 7.45(\mathrm{~d}, J=8.2 \mathrm{~Hz}, 1 \mathrm{H}), 6.47(\mathrm{~d}, J=8.5 \mathrm{~Hz}, 1 \mathrm{H})$, 5.77 (ddd, $J=8.2,2.4,0.6 \mathrm{~Hz}, 1 \mathrm{H}$ ), 5.60 (dd, $J=8.5,5.1 \mathrm{~Hz}, 1 \mathrm{H}), 5.33(\mathrm{~d}, J=5.1 \mathrm{~Hz}, 1 \mathrm{H}$ ), 3.89 (dd, $J=7.5,5.4 \mathrm{~Hz}, 1 \mathrm{H}$ ), 2.69 (hept, $J=7.0 \mathrm{~Hz}, 1 \mathrm{H}), 2.63-2.49(\mathrm{~m}, 2 \mathrm{H}), 1.27-1.21(\mathrm{~m}, 8 \mathrm{H})$, $1.17(\mathrm{~d}, J=7.0 \mathrm{~Hz}, 3 \mathrm{H}), 1.13(\mathrm{~d}, J=7.1 \mathrm{~Hz}, 3 \mathrm{H}), 1.13(\mathrm{~d}, J=7.1 \mathrm{~Hz}, 3 \mathrm{H}), 1.12(\mathrm{~d}, J=6.8 \mathrm{~Hz}$, $3 \mathrm{H})$.

${ }^{13} \mathrm{C}$ NMR (126 MHz, CDCl $)$ $\delta$ (ppm) 178.6, 176.1, 175.5, 162.1, 150.4, 139.7, 103.5, 84.2, 73.0, $71.8,66.5,53.5,34.0,33.6,33.6,19.0,18.8,18.7,18.7,18.6,12.7$.

IR (ATR): $\tilde{v}\left(\mathrm{~cm}^{-1}\right)=3195,2977,2938,1694,1463,1383,1297,1256,1190,1146,1099$.

HR-MS (ESI): $m / z$ calcd for $\mathrm{C}_{22} \mathrm{H}_{30} \mathrm{~N}_{2} \mathrm{O}_{9} \mathrm{Na}^{+}:$:89.18435; found: 489.18342 .

$[\alpha]_{\mathrm{D}}^{20}=-19.5^{\circ}\left(\mathrm{c}=0.78, \mathrm{CHCl}_{3}\right)$. 


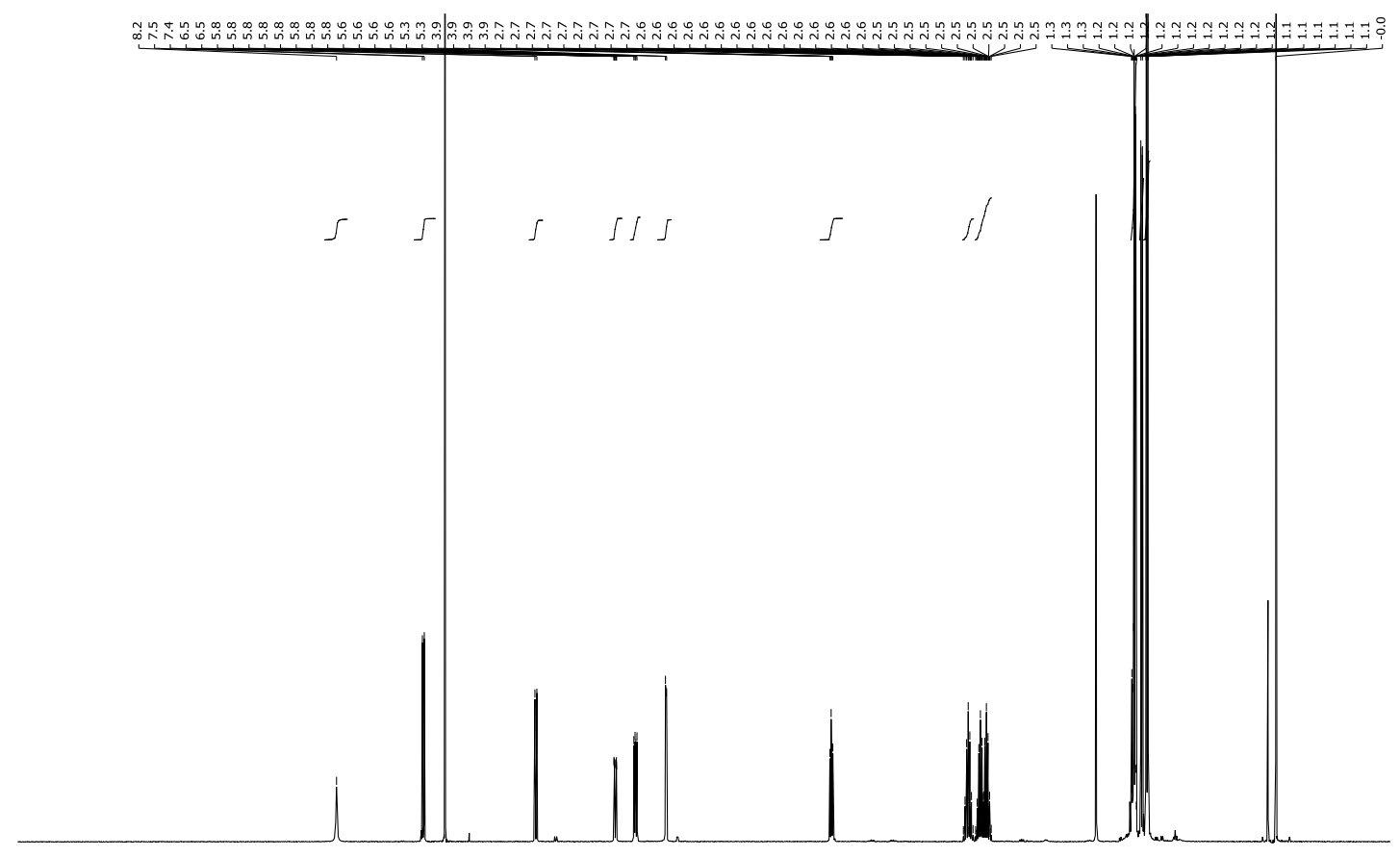

\begin{tabular}{llllllllllllllllllllllllllllll}
\hline 1.0 & 10.5 & 10.0 & 9.5 & 9.0 & 8.5 & 8.0 & 7.5 & 7.0 & 6.5 & 6.0 & 5.5 & $\begin{array}{c}5.0 \\
\mathrm{f} 1(\mathrm{ppm})\end{array}$ & 4.5 & 4.0 & 3.5 & 3.0 & 2.5 & 2.0 & 1.5 & 1.0 & 0.5 & 0.0 & -0.5 & -1
\end{tabular}

Figure S20. ${ }^{1} \mathrm{H}$ NMR $\left(500 \mathrm{MHz}, \mathrm{CDCl}_{3}\right)$ spectrum of compound 20.

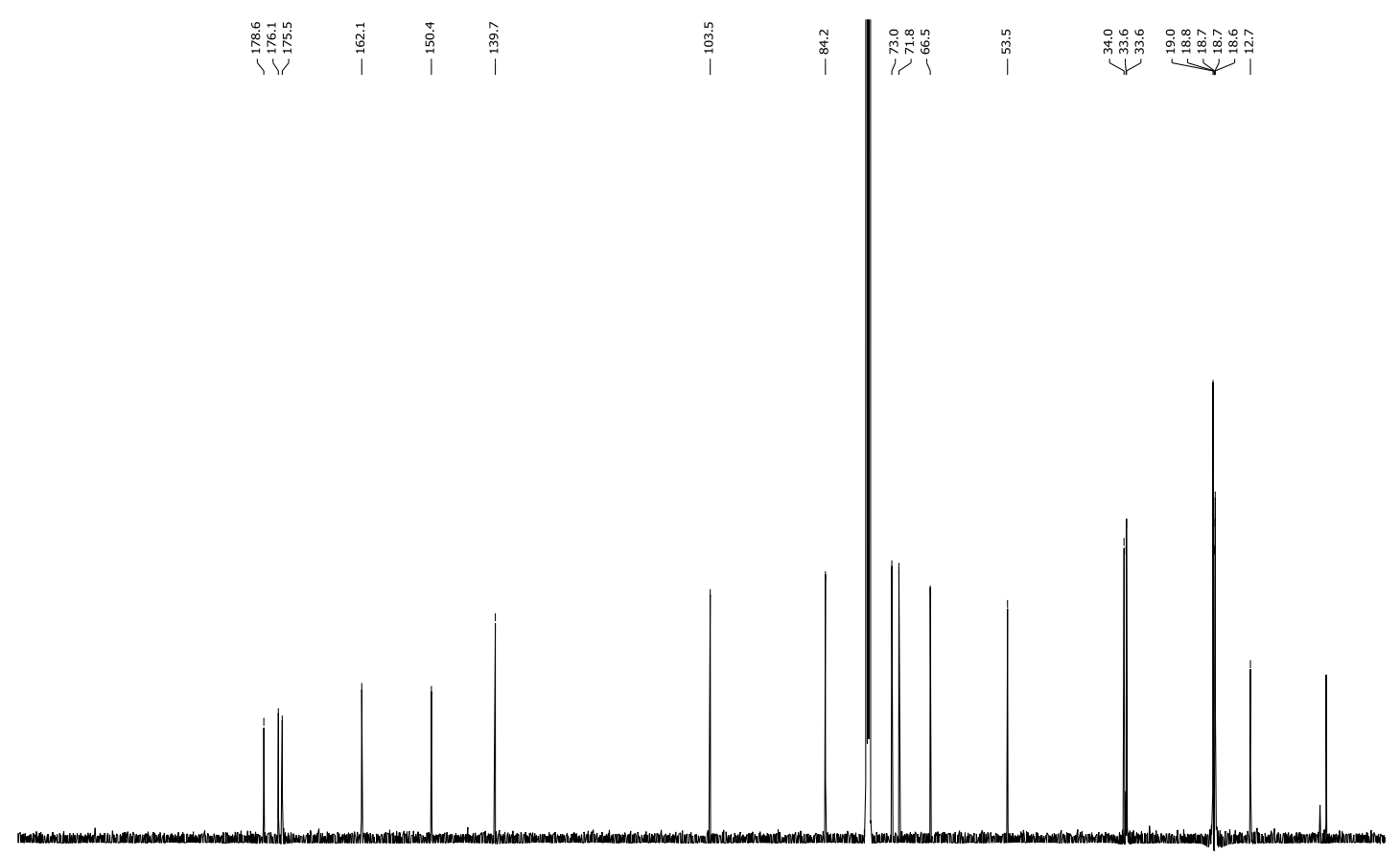

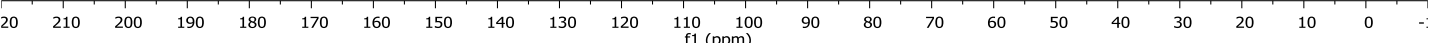

Figure S21. ${ }^{13} \mathrm{C}$ NMR (126 MHz, $\mathrm{CDCl}_{3}$ ) spectrum of compound 20. 


\section{Compound 21}

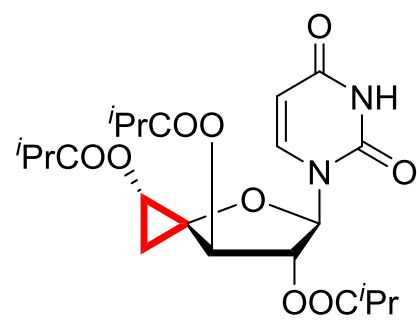

Uridine derivative 19 (4.20 mg, $12.8 \mu \mathrm{mol}, 1.00$ eq.) was dissolved in pyridine $(0.50 \mathrm{~mL})$ and isobutyric anhydride (0.01 mL, $4.38 \mathrm{mmol}, 30.0$ eq.) and DMAP (0.50 mg, $14.7 \mu \mathrm{mol}, 0.34$ eq.) were added. The reaction mixture was stirred for $16 \mathrm{~h}$ at room temperature and finally DCM $(5.00 \mathrm{~mL})$ and a saturated aqueous solution of $\mathrm{NaHCO}_{3}(2.00 \mathrm{~mL})$ were added. The aqueous phase was extracted with DCM $(3 \mathrm{x}, 5.00 \mathrm{~mL})$, the combined organic phases were dried over $\mathrm{Na}_{2} \mathrm{SO}_{4}$, filtered and evaporated to dryness. Column purification $\left(\mathrm{SiO}_{2}\right.$, pentane/ethyl acetate, 3:1 to $1: 1)$ afforded compound 21 (3.50 mg, $7.50 \mu \mathrm{mol}, 59 \%$ yield) as a colorless oil.

For biological investigations compound 21 was previously purified by HPLC (C18, isocratic, $\left.\mathrm{CH}_{3} \mathrm{CN}: \mathrm{H}_{2} \mathrm{O}, 70: 30, t_{\mathrm{R}}=6.25 \mathrm{~min}\right)$.

${ }^{1} \mathrm{H}$ NMR $\left(300 \mathrm{MHz}, \mathrm{CDCl}_{3}\right) \delta(\mathrm{ppm}) 8.25(\mathrm{~s}, 1 \mathrm{H}), 7.54(\mathrm{~d}, J=8.2 \mathrm{~Hz}, 1 \mathrm{H}), 6.15(\mathrm{~d}, J=2.0 \mathrm{~Hz}, 1 \mathrm{H})$, $5.74(\mathrm{dd}, J=8.2,1.7 \mathrm{~Hz}, 1 \mathrm{H}), 5.32(\mathrm{dd}, J=2.0,1.5 \mathrm{~Hz}, 1 \mathrm{H}), 4.95(\mathrm{~d}, J=1.5 \mathrm{~Hz}, 1 \mathrm{H}), 4.11$ (t, $J=$ $6.5 \mathrm{~Hz}, 1 \mathrm{H}), 2.74-2.43(\mathrm{~m}, 3 \mathrm{H}), 1.36(\mathrm{~d}, J=6.5 \mathrm{~Hz}, 2 \mathrm{H}), 1.25-1.18(\mathrm{~m}, 12 \mathrm{H}), 1.15(\mathrm{~d}, J=7.0$ $\mathrm{Hz}, 3 \mathrm{H}), 1.11(\mathrm{~d}, \mathrm{~J}=7.0 \mathrm{~Hz}, 3 \mathrm{H})$.

${ }^{13} \mathrm{C}$ NMR (126 MHz, $\left.\mathrm{CDCl}_{3}\right) \delta$ (ppm) 178.0, 176.2, 162.6, 149.7, 149.7, 140.0, 102.0, 88.5, 80.3, $69.2,50.4$, 33.9, 33.7, 33.6, 18.9, 18.9, 18.8, 18.7, 18.6, 17.0.

IR (ATR): $\tilde{v}\left(\mathrm{~cm}^{-1}\right)=3198,2976,2929,1743,1695,1461,1384,1257,1196,1147,1111$.

HR-MS (ESI): $m / z$ calcd for $\mathrm{C}_{22} \mathrm{H}_{30} \mathrm{~N}_{2} \mathrm{O}_{9} \mathrm{Na}^{+}:$: 489.18435 ; found: 489.18375 .

$[\alpha]_{\mathrm{D}}^{20}=32.7^{\circ}\left(\mathrm{c}=0.35, \mathrm{CHCl}_{3}\right)$. 

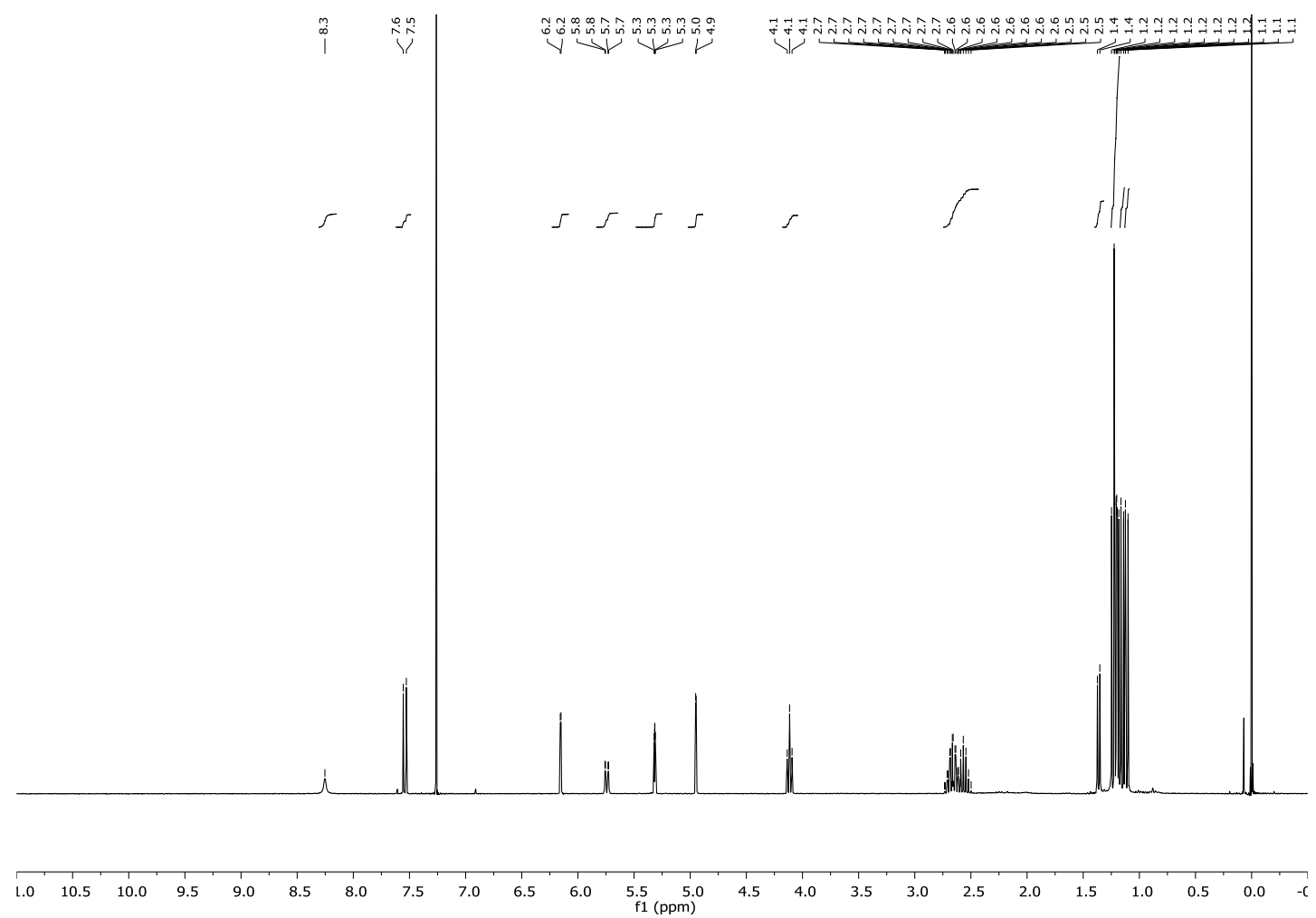

Figure S22. ${ }^{1} \mathrm{H} \mathrm{NMR}\left(300 \mathrm{MHz}, \mathrm{CDCl}_{3}\right)$ of compound 21.
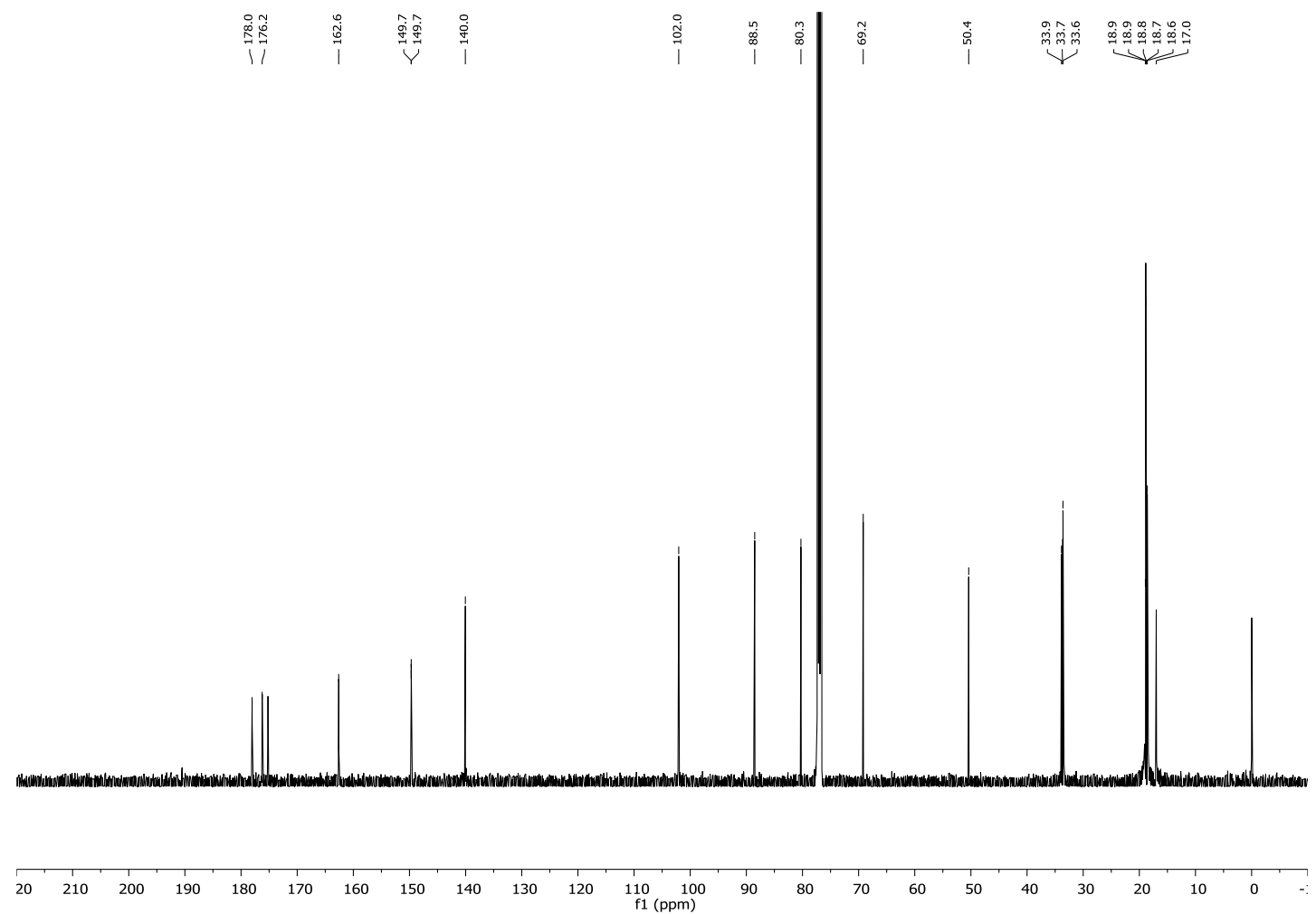

Figure S23. ${ }^{13} \mathrm{C}$ NMR $\left(126 \mathrm{MHz}, \mathrm{CDCl}_{3}\right)$ of compound 21. 


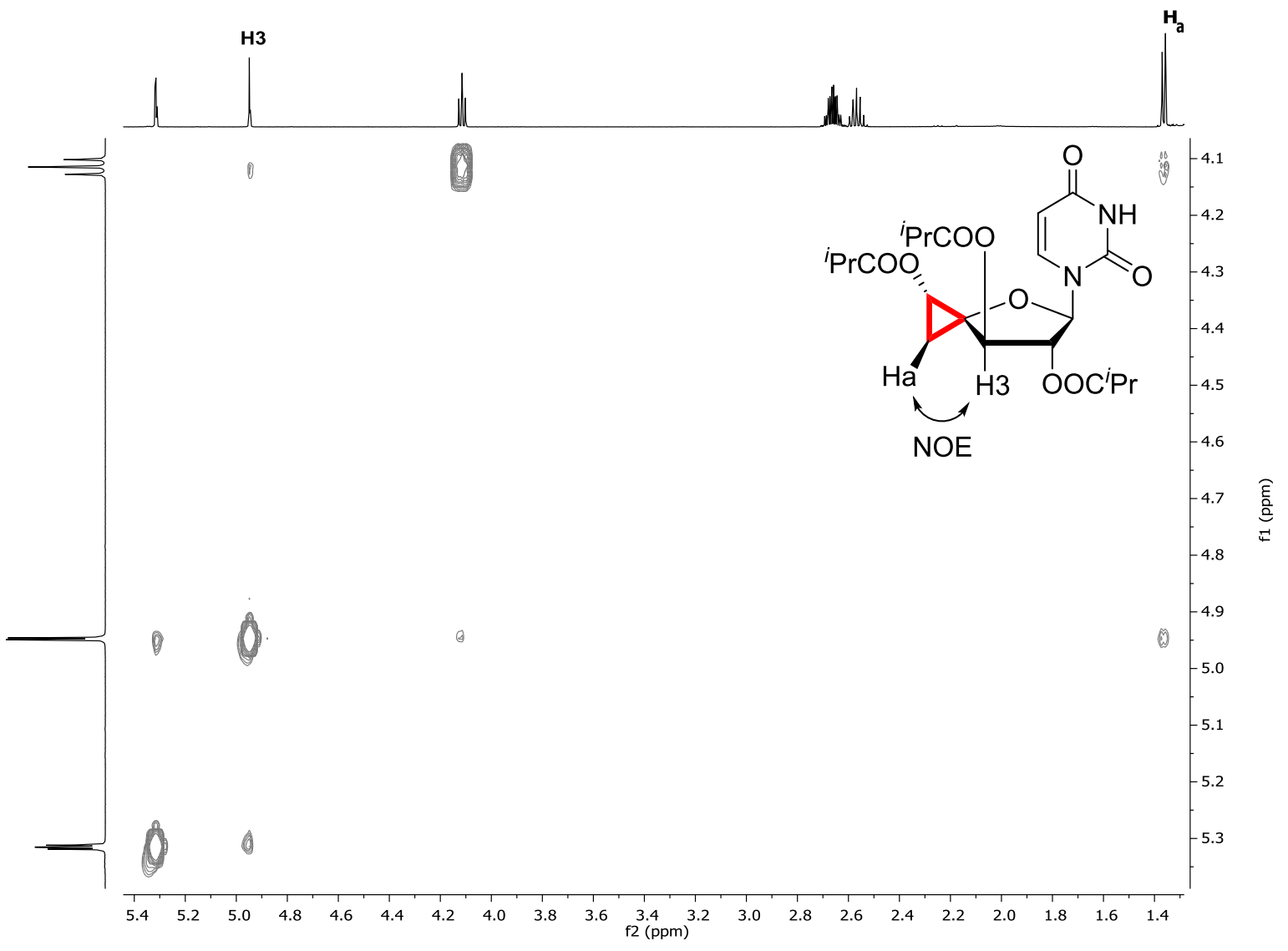

Figure S24. Partial NOESY (300 MHz, $\left.\mathrm{CDCl}_{3}\right)$ of compound 21. 


\section{X-Ray Crystallographic Data}

\section{X-Ray Data for Compound 6}

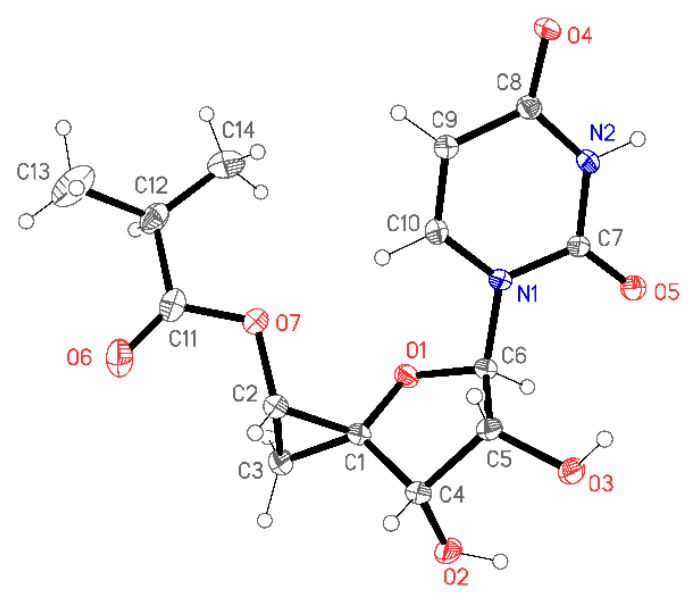

Table 1. Crystal data and structure refinement.

Identification code

Empirical formula

Formula weight

Temperature

Wavelength

Crystal system

Space group

Unit cell dimensions

Volume

Z

Density (calculated)

Absorption coefficient

$F(000)$

Crystal size

Theta range for data collection

Index ranges

Reflections collected cholerb

$\mathrm{C}_{14} \mathrm{H}_{18} \mathrm{~N}_{2} \mathrm{O}_{7}$

326.30

100(2) K

$1.54184 \AA$

orthorhombic

$P 22_{1} 2_{1}$

$a=5.00217(8) \AA \quad \alpha=90^{\circ}$

$b=8.12138(10) \AA \quad \beta=90^{\circ}$

c $=37.1173(5) \AA \quad Y=90^{\circ}$

1507.87(4) $\AA^{3}$

4

$1.437 \mathrm{Mg} / \mathrm{m}^{3}$

$0.993 \mathrm{~mm}^{-1}$

688

$0.15 \times 0.15 \times 0.02 \mathrm{~mm}^{3}$

2.381 to $77.516^{\circ}$.

$-6<=\mathrm{h}<=6,-10<=\mathrm{k}<=10,-46<=\mathrm{k}<=46$

31690 
Independent reflections

Completeness to theta $=75.000^{\circ}$

Absorption correction

Max. and min. transmission

Refinement method

Data / restraints / parameters

Goodness-of-fit on $\mathrm{F}^{2}$

Final $R$ indices [l>2sigma(I)]

$\mathrm{R}$ indices (all data)

Absolute structure parameter

Largest diff. peak and hole
$3184[R($ int $)=0.0379]$

$99.9 \%$

Semi-empirical from equivalents

1.00000 and 0.70878

Full-matrix least-squares on $\mathrm{F}^{2}$

3184 / 24 / 235

1.072

$\mathrm{R} 1=0.0326, w R 2=0.0867$

$\mathrm{R} 1=0.0329, \mathrm{wR} 2=0.0869$

0.07(4)

0.300 and -0.218 e. $\AA^{-3}$ 
Table 2. Atomic coordinates $\left(\times 10^{4}\right)$ and equivalent isotropic displacement parameters $\left(\AA^{2} \times 10^{3}\right)$. $\mathrm{U}(\mathrm{eq})$ is defined as one third of the trace of the orthogonalized $\mathrm{U}^{\mathrm{ij}}$ tensor.

\begin{tabular}{|c|c|c|c|c|}
\hline & $x$ & $y$ & $z$ & $U(e q)$ \\
\hline$C(1)$ & $2032(4)$ & $650(2)$ & $1302(1)$ & $16(1)$ \\
\hline $\mathrm{C}(2)$ & $4249(4)$ & $804(2)$ & $1572(1)$ & $19(1)$ \\
\hline$C(3)$ & $1517(4)$ & $219(3)$ & $1686(1)$ & $21(1)$ \\
\hline$C(4)$ & $2151(4)$ & $-598(2)$ & $1005(1)$ & $18(1)$ \\
\hline$C(5)$ & $2796(4)$ & $513(2)$ & $685(1)$ & $16(1)$ \\
\hline$C(6)$ & $1248(4)$ & 2096(2) & $778(1)$ & $16(1)$ \\
\hline $\mathrm{N}(1)$ & 2695(3) & $3607(2)$ & $676(1)$ & $16(1)$ \\
\hline $\mathrm{N}(2)$ & $3459(4)$ & $5796(2)$ & $284(1)$ & $17(1)$ \\
\hline$C(7)$ & $1972(4)$ & $4418(2)$ & $362(1)$ & $15(1)$ \\
\hline $\mathrm{C}(8)$ & $5655(4)$ & $6356(2)$ & $470(1)$ & $16(1)$ \\
\hline $\mathrm{C}(9)$ & $6260(4)$ & $5464(2)$ & $797(1)$ & $18(1)$ \\
\hline$C(10)$ & $4795(4)$ & $4136(2)$ & $882(1)$ & $18(1)$ \\
\hline$C(11)$ & 5883(5) & $2646(3)$ & $2008(1)$ & $26(1)$ \\
\hline$C(12)$ & $6470(7)$ & $4416(4)$ & $2111(1)$ & $26(1)$ \\
\hline$C(13)$ & $9075(9)$ & $4443(6)$ & $2322(2)$ & $49(1)$ \\
\hline$C(14)$ & $5801(13)$ & $5703(5)$ & $1839(1)$ & $50(1)$ \\
\hline$C\left(12^{\prime}\right)$ & $7500(30)$ & $4306(14)$ & $1979(4)$ & $29(3)$ \\
\hline$C\left(13^{\prime}\right)$ & $9630(30)$ & $4594(18)$ & $2165(4)$ & $35(3)$ \\
\hline$C\left(14^{\prime}\right)$ & $7200(30)$ & $5458(16)$ & $1756(4)$ & $34(3)$ \\
\hline $\mathrm{O}(1)$ & $851(3)$ & $2107(2)$ & $1158(1)$ & $17(1)$ \\
\hline $\mathrm{O}(2)$ & $-389(4)$ & $-1331(2)$ & $969(1)$ & $27(1)$ \\
\hline $\mathrm{O}(3)$ & 1933(3) & $-192(2)$ & $360(1)$ & $21(1)$ \\
\hline $\mathrm{O}(4)$ & $6971(3)$ & $7541(2)$ & $359(1)$ & $20(1)$ \\
\hline $\mathrm{O}(5)$ & 127(3) & $3945(2)$ & $171(1)$ & $21(1)$ \\
\hline $\mathrm{O}(6)$ & $6094(4)$ & $1532(2)$ & $2214(1)$ & $34(1)$ \\
\hline $\mathrm{O}(7)$ & $4926(3)$ & $2447(2)$ & $1667(1)$ & $22(1)$ \\
\hline
\end{tabular}


Table 3. Bond lengths $[\AA ̊]$ and angles $\left[^{\circ}\right]$.

\begin{tabular}{|c|c|c|c|}
\hline $\mathrm{C}(1)-\mathrm{O}(1)$ & $1.426(2)$ & $N(1)-C(7)$ & $1.387(2)$ \\
\hline$C(1)-C(3)$ & $1.492(3)$ & $\mathrm{N}(2)-\mathrm{C}(7)$ & $1.374(2)$ \\
\hline$C(1)-C(4)$ & $1.497(3)$ & $\mathrm{N}(2)-\mathrm{C}(8)$ & $1.376(3)$ \\
\hline$C(1)-C(2)$ & $1.502(3)$ & $\mathrm{C}(7)-\mathrm{O}(5)$ & $1.224(2)$ \\
\hline $\mathrm{C}(2)-\mathrm{O}(7)$ & $1.421(2)$ & $\mathrm{C}(8)-\mathrm{O}(4)$ & $1.237(2)$ \\
\hline$C(2)-C(3)$ & $1.507(3)$ & $\mathrm{C}(8)-\mathrm{C}(9)$ & $1.444(3)$ \\
\hline $\mathrm{C}(4)-\mathrm{O}(2)$ & $1.410(3)$ & $C(9)-C(10)$ & $1.342(3)$ \\
\hline$C(4)-C(5)$ & $1.526(3)$ & $\mathrm{C}(11)-\mathrm{O}(6)$ & $1.190(3)$ \\
\hline $\mathrm{C}(5)-\mathrm{O}(3)$ & $1.402(2)$ & $\mathrm{C}(11)-\mathrm{O}(7)$ & $1.362(2)$ \\
\hline$C(5)-C(6)$ & $1.539(3)$ & $C(11)-C(12)$ & $1.516(4)$ \\
\hline $\mathrm{C}(6)-\mathrm{O}(1)$ & $1.426(2)$ & $C(12)-C(14)$ & $1.489(5)$ \\
\hline $\mathrm{C}(6)-\mathrm{N}(1)$ & $1.474(2)$ & $C(12)-C(13)$ & $1.520(5)$ \\
\hline$N(1)-C(10)$ & $1.369(3)$ & & \\
\hline $\mathrm{O}(1)-\mathrm{C}(1)-\mathrm{C}(3)$ & $118.72(17)$ & $C(10)-N(1)-C(7)$ & $121.42(16)$ \\
\hline $\mathrm{O}(1)-\mathrm{C}(1)-\mathrm{C}(4)$ & $107.65(15)$ & $C(10)-N(1)-C(6)$ & $119.62(16)$ \\
\hline$C(3)-C(1)-C(4)$ & 123.48(17) & $C(7)-N(1)-C(6)$ & $118.88(15)$ \\
\hline$O(1)-C(1)-C(2)$ & $119.14(16)$ & $\mathrm{C}(7)-\mathrm{N}(2)-\mathrm{C}(8)$ & $126.57(16)$ \\
\hline$C(3)-C(1)-C(2)$ & $60.46(13)$ & $\mathrm{O}(5)-\mathrm{C}(7)-\mathrm{N}(2)$ & $122.83(17)$ \\
\hline$C(4)-C(1)-C(2)$ & $121.27(18)$ & $\mathrm{O}(5)-\mathrm{C}(7)-\mathrm{N}(1)$ & $122.22(17)$ \\
\hline $\mathrm{O}(7)-\mathrm{C}(2)-\mathrm{C}(1)$ & $114.84(17)$ & $N(2)-C(7)-N(1)$ & $114.95(16)$ \\
\hline $\mathrm{O}(7)-\mathrm{C}(2)-\mathrm{C}(3)$ & 116.26(18) & $\mathrm{O}(4)-\mathrm{C}(8)-\mathrm{N}(2)$ & $120.93(17)$ \\
\hline$C(1)-C(2)-C(3)$ & $59.44(13)$ & $\mathrm{O}(4)-\mathrm{C}(8)-\mathrm{C}(9)$ & $124.00(19)$ \\
\hline$C(1)-C(3)-C(2)$ & $60.10(13)$ & $N(2)-C(8)-C(9)$ & $115.07(17)$ \\
\hline $\mathrm{O}(2)-\mathrm{C}(4)-\mathrm{C}(1)$ & 108.65(17) & $C(10)-C(9)-C(8)$ & $119.13(18)$ \\
\hline $\mathrm{O}(2)-\mathrm{C}(4)-\mathrm{C}(5)$ & $111.49(16)$ & $C(9)-C(10)-N(1)$ & $122.63(17)$ \\
\hline$C(1)-C(4)-C(5)$ & $100.41(15)$ & $\mathrm{O}(6)-\mathrm{C}(11)-\mathrm{O}(7)$ & $122.6(2)$ \\
\hline $\mathrm{O}(3)-\mathrm{C}(5)-\mathrm{C}(4)$ & $111.24(15)$ & $\mathrm{O}(6)-\mathrm{C}(11)-\mathrm{C}(12)$ & $122.8(2)$ \\
\hline $\mathrm{O}(3)-\mathrm{C}(5)-\mathrm{C}(6)$ & $112.22(16)$ & $\mathrm{O}(7)-\mathrm{C}(11)-\mathrm{C}(12)$ & $114.5(2)$ \\
\hline$C(4)-C(5)-C(6)$ & 102.32(15) & $C(14)-C(12)-C(11)$ & $116.9(2)$ \\
\hline $\mathrm{O}(1)-\mathrm{C}(6)-\mathrm{N}(1)$ & $108.49(15)$ & $C(14)-C(12)-C(13)$ & $122.0(4)$ \\
\hline $\mathrm{O}(1)-\mathrm{C}(6)-\mathrm{C}(5)$ & $107.25(14)$ & $C(11)-C(12)-C(13)$ & 108.0(3) \\
\hline$N(1)-C(6)-C(5)$ & $113.00(16)$ & $\mathrm{C}(6)-\mathrm{O}(1)-\mathrm{C}(1)$ & $107.88(14)$ \\
\hline
\end{tabular}


115.19(16) 
Table 4. Torsion angles $\left[^{\circ}\right]$

\begin{tabular}{|c|c|c|c|}
\hline $\mathrm{O}(1)-\mathrm{C}(1)-\mathrm{C}(2)-\mathrm{O}(7)$ & $1.4(3)$ & $\mathrm{C}(8)-\mathrm{N}(2)-\mathrm{C}(7)-\mathrm{O}(5)$ & $-175.84(19)$ \\
\hline$C(3)-C(1)-C(2)-O(7)$ & $-107.0(2)$ & $\mathrm{C}(8)-\mathrm{N}(2)-\mathrm{C}(7)-\mathrm{N}(1)$ & $4.9(3)$ \\
\hline$C(4)-C(1)-C(2)-O(7)$ & 139.54(18) & $\mathrm{C}(10)-\mathrm{N}(1)-\mathrm{C}(7)-\mathrm{O}(5)$ & 178.82(19) \\
\hline$O(1)-C(1)-C(2)-C(3)$ & $108.4(2)$ & $\mathrm{C}(6)-\mathrm{N}(1)-\mathrm{C}(7)-\mathrm{O}(5)$ & $2.0(3)$ \\
\hline$C(4)-C(1)-C(2)-C(3)$ & $-113.4(2)$ & $\mathrm{C}(10)-\mathrm{N}(1)-\mathrm{C}(7)-\mathrm{N}(2)$ & $-1.9(3)$ \\
\hline $\mathrm{O}(1)-\mathrm{C}(1)-\mathrm{C}(3)-\mathrm{C}(2)$ & $-109.13(19)$ & $\mathrm{C}(6)-\mathrm{N}(1)-\mathrm{C}(7)-\mathrm{N}(2)$ & $-178.80(17)$ \\
\hline$C(4)-C(1)-C(3)-C(2)$ & $109.9(2)$ & $\mathrm{C}(7)-\mathrm{N}(2)-\mathrm{C}(8)-\mathrm{O}(4)$ & 174.16(19) \\
\hline $\mathrm{O}(7)-\mathrm{C}(2)-\mathrm{C}(3)-\mathrm{C}(1)$ & 104.63(19) & $\mathrm{C}(7)-\mathrm{N}(2)-\mathrm{C}(8)-\mathrm{C}(9)$ & $-5.9(3)$ \\
\hline $\mathrm{O}(1)-\mathrm{C}(1)-\mathrm{C}(4)-\mathrm{O}(2)$ & $-80.10(18)$ & $\mathrm{O}(4)-\mathrm{C}(8)-\mathrm{C}(9)-\mathrm{C}(10)$ & $-176.2(2)$ \\
\hline$C(3)-C(1)-C(4)-O(2)$ & $64.5(2)$ & $N(2)-C(8)-C(9)-C(10)$ & $3.9(3)$ \\
\hline $\mathrm{C}(2)-\mathrm{C}(1)-\mathrm{C}(4)-\mathrm{O}(2)$ & 137.62(18) & $\mathrm{C}(8)-\mathrm{C}(9)-\mathrm{C}(10)-\mathrm{N}(1)$ & $-1.5(3)$ \\
\hline$O(1)-C(1)-C(4)-C(5)$ & $37.0(2)$ & $C(7)-N(1)-C(10)-C(9)$ & $0.5(3)$ \\
\hline$C(3)-C(1)-C(4)-C(5)$ & $-178.45(19)$ & $C(6)-N(1)-C(10)-C(9)$ & $177.35(19)$ \\
\hline$C(2)-C(1)-C(4)-C(5)$ & $-105.30(19)$ & $\mathrm{O}(6)-\mathrm{C}(11)-\mathrm{C}(12)-\mathrm{C}(14)$ & $-172.7(4)$ \\
\hline $\mathrm{O}(2)-\mathrm{C}(4)-\mathrm{C}(5)-\mathrm{O}(3)$ & $-40.0(2)$ & $\mathrm{O}(7)-\mathrm{C}(11)-\mathrm{C}(12)-\mathrm{C}(14)$ & $3.2(4)$ \\
\hline$C(1)-C(4)-C(5)-O(3)$ & $-154.96(17)$ & $\mathrm{O}(6)-\mathrm{C}(11)-\mathrm{C}(12)-\mathrm{C}(13)$ & $45.1(4)$ \\
\hline $\mathrm{O}(2)-\mathrm{C}(4)-\mathrm{C}(5)-\mathrm{C}(6)$ & $80.00(18)$ & $\mathrm{O}(7)-\mathrm{C}(11)-\mathrm{C}(12)-\mathrm{C}(13)$ & $-139.0(3)$ \\
\hline$C(1)-C(4)-C(5)-C(6)$ & $-34.95(19)$ & $N(1)-C(6)-O(1)-C(1)$ & $-122.57(16)$ \\
\hline $\mathrm{O}(3)-\mathrm{C}(5)-\mathrm{C}(6)-\mathrm{O}(1)$ & $142.20(16)$ & $\mathrm{C}(5)-\mathrm{C}(6)-\mathrm{O}(1)-\mathrm{C}(1)$ & $-0.2(2)$ \\
\hline$C(4)-C(5)-C(6)-O(1)$ & $22.87(19)$ & $\mathrm{C}(3)-\mathrm{C}(1)-\mathrm{O}(1)-\mathrm{C}(6)$ & $-170.15(17)$ \\
\hline $\mathrm{O}(3)-\mathrm{C}(5)-\mathrm{C}(6)-\mathrm{N}(1)$ & $-98.29(19)$ & $C(4)-C(1)-O(1)-C(6)$ & $-23.6(2)$ \\
\hline$C(4)-C(5)-C(6)-N(1)$ & $142.38(16)$ & $\mathrm{C}(2)-\mathrm{C}(1)-\mathrm{O}(1)-\mathrm{C}(6)$ & 119.62(19) \\
\hline $\mathrm{O}(1)-\mathrm{C}(6)-\mathrm{N}(1)-\mathrm{C}(10)$ & $42.7(2)$ & $\mathrm{O}(6)-\mathrm{C}(11)-\mathrm{O}(7)-\mathrm{C}(2)$ & $-1.3(3)$ \\
\hline$C(5)-C(6)-N(1)-C(10)$ & $-76.1(2)$ & $C(12)-C(11)-O(7)-C(2)$ & $-177.2(2)$ \\
\hline $\mathrm{O}(1)-\mathrm{C}(6)-\mathrm{N}(1)-\mathrm{C}(7)$ & $-140.40(17)$ & $C(1)-C(2)-O(7)-C(11)$ & $150.14(18)$ \\
\hline$C(5)-C(6)-N(1)-C(7)$ & $100.81(19)$ & $\mathrm{C}(3)-\mathrm{C}(2)-\mathrm{O}(7)-\mathrm{C}(11)$ & $83.5(2)$ \\
\hline
\end{tabular}


Table 5. Hydrogen bonds for cholerb [ A $^{\text {and }}{ }^{\circ}$ ].

\begin{tabular}{lccll}
\hline $\mathrm{D}-\mathrm{H} \ldots \mathrm{A}$ & $\mathrm{d}(\mathrm{D}-\mathrm{H})$ & $\mathrm{d}(\mathrm{H} \ldots \mathrm{A})$ & $\mathrm{d}(\mathrm{D} \ldots \mathrm{A})$ & $<(\mathrm{DHA})$ \\
\hline $\mathrm{N}(2)-\mathrm{H}(01) \ldots \mathrm{O}(4) \# 1$ & $0.90(3)$ & $1.94(3)$ & $2.841(2)$ & $178(3)$ \\
$\mathrm{O}(2)-\mathrm{H}(02) \ldots \mathrm{O}(3)$ & $0.90(3)$ & $2.15(3)$ & $2.704(2)$ & $119(2)$ \\
$\mathrm{O}(2)-\mathrm{H}(02) \ldots \mathrm{O}(4) \# 2$ & $0.90(3)$ & $2.00(3)$ & $2.777(2)$ & $143(3)$ \\
$\mathrm{O}(3)-\mathrm{H}(03) \ldots \mathrm{O}(5) \# 3$ & $0.92(3)$ & $1.82(3)$ & $2.733(2)$ & $172(3)$ \\
$\mathrm{C}(10)-\mathrm{H}(10) \ldots \mathrm{O}(7)$ & 0.95 & 2.31 & $3.222(2)$ & 160.7 \\
$\mathrm{C}(12)-\mathrm{H}(12) \ldots \mathrm{O}(6) \# 4$ & 1.00 & 2.44 & $3.298(4)$ & 143.2 \\
$\mathrm{C}(3)-\mathrm{H}(3 \mathrm{~B}) \ldots \mathrm{O}(6) \# 5$ & 0.99 & 2.62 & $3.513(3)$ & 149.7 \\
\hline
\end{tabular}

Symmetry transformations used to generate equivalent atoms:

$\# 1 \mathrm{x}-1 / 2,-y+3 / 2,-z$

$\# 2 \mathrm{x}-1, \mathrm{y}-1, \mathrm{z}$

$\# 3 x+1 / 2,-y+1 / 2,-z$

$\# 4-x+1, y+1 / 2,-z+1 / 2$

$\# 5 x-1, y, z$ 


\section{Antiviral Investigations}

\section{Materials and Methods}

\section{Cell line, medium and virus}

HEp2 cells were purchased from the ATCC (CCL-23) and cultured in advanced MEM medium (Thermo Fisher Scientific; Waltham, MA) supplemented with $10 \%$ heat inactivated FCS (Capricorn Scientific; Ebsdorfergrund, Germany), 2 mM L-Glutamine (Thermo Fisher Scientific; Waltham, MA), $100 \mathrm{U} / \mathrm{ml}$ Penicillin and Streptomycin (Thermo Fisher Scientific; Waltham, MA) and $1 \times$ non-essential amino acids (Thermo Fisher Scientific; Waltham, MA). Cells were grown at $37^{\circ} \mathrm{C}$ in humidified air containing $5 \% \mathrm{CO}_{2}$. Recombinant HRSV-A-Luc luciferase reporter virus was kindly provided by Jean-François Éléöuet. ${ }^{[3]}$

\section{MTT assay}

$1,5 \times 10^{4} \mathrm{HEp} 2$ cells/well were seeded in 96 well plates. $24 \mathrm{~h}$ later, supernatant was changed to $100 \mu \mathrm{l}$ fresh medium containing 1\% DMSO only, the indicated compound or ribavirin (SigmaAldrich, St. Louis, MO) concentration or $10 \mu \mathrm{M}$ puromycin. After additional $24 \mathrm{~h}$, supernatant was changed to prewarmed medium containing $0.5 \mathrm{mg} / \mathrm{ml}$ Thiazolyl Blue tetrazolium bromide (MTT) (Sigma-Aldrich, St. Louis, MO) and the cells were incubated for further $30 \mathrm{~min}$. Medium was removed and cells were lysed in $50 \mu$ of isopropanol. Cell metabolism induced color change was measured at $570 \mathrm{~nm}$ and $630 \mathrm{~nm}$ in a Biotek Synergy 2 multi-detection microplate reader. The unspecific $630 \mathrm{~nm}$ value was subtracted and cell viability was set to $0 \%$ for the puromycin control and normalized to the DMSO control (100\%).Infection assay

Cells were seeded as stated above. $24 \mathrm{~h}$ later, cells were infected with recombinant HRSV-A-Luc at a multiplicity of infection of 0.1 in presence of $1 \%$ DMSO only or the indicated compound or ribavirin concentration. After further $24 \mathrm{~h}$, cells were washed with PBS once, lysed in $35 \mu$ l fireflyluciferase lysis buffer (1\% Triton X-100; 25 mM Gly-Gly; 15 mM MgSO $; 4$ mM EGTA; 1 mM DTT) and frozen at $-20^{\circ} \mathrm{C} .20 \mu \mathrm{l}$ of thawed cell lysate was mixed with $72 \mu \mathrm{l}$ of assay buffer ( $25 \mathrm{mM}$ GlyGly; 13.7 mM K2 $\mathrm{HPO}_{4} ; 1.28$ mM KH $\mathrm{PO}_{4} ; 15$ mM MgSO 4 ; 4 mM EGTA; 1 mM DTT; 2 mM ATP) and $40 \mu \mathrm{l}$ of substrate (0,2 mM D-luciferin; $25 \mathrm{mM}$ Gly-Gly). Luciferase activity was measured in relative light units (RLU) using a Berthold Centro XS ${ }^{3}$ LB 960 microplate luminometer. Background counts of uninfected cells were subtracted and luciferase activity, representing viral infection, was normalized to the DMSO control (100\%). 


\section{Curve fit}

Dose response curves were generated using XY analysis in GraphPad Prism 8 (Nonlinear regression (curve fit); Dose-response-Inhibition; Log(inhibitor) vs. Response - variable slope (four parameters); Bottom constant equal to 0; Top constant equal to 100). 


\section{Conformational Investigations}

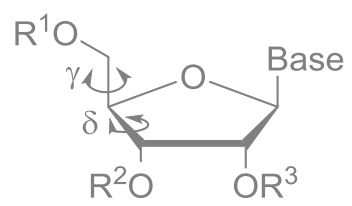

Table 6. Selected torsion angles for compound 6 and various modified polynucleotides determined by crystal data analysis.

\begin{tabular}{|c|c|c|c|c|c|c|c|c|c|}
\hline$\theta\left[{ }^{\circ}\right]$ & $\mathrm{HO} \mathrm{OH}$ & joe & 烈 & \multicolumn{2}{|c|}{$1\left(U_{1}\right)$} & \multicolumn{2}{|c|}{$2\left(U_{9}\right)$} & \multicolumn{2}{|c|}{$3\left(U_{12}\right)$} \\
\hline$r$ & 139.5 & 54.9 & 151 & 57.6 & 73.1 & 63.8 & 61.3 & 93.9 & 72.3 \\
\hline$\delta$ & 137.6 & 64.0 & 102 & 80.0 & 76.6 & 81.4 & 68.9 & 135.6 & 80.8 \\
\hline $\mathrm{pdb}$ & & $3 U K B$ & & \multicolumn{2}{|c|}{$1 S D R$} & \multicolumn{2}{|c|}{ 1SDR } & \multicolumn{2}{|c|}{ 1SDR } \\
\hline Rof & & & {$[5]$} & \multicolumn{2}{|c|}{$[6]$} & & {$[6$} & \\
\hline
\end{tabular}

1SDR: RNA dodecamer containing the Escherichia coli Shine-Dalgarno sequence.

$1 \quad 5^{\prime}-R\left(U_{1} A_{2} A_{3} G_{4} G_{5} A_{6} G_{7} G_{8} U_{9} G_{10} A_{11} U_{12}\right)-3^{\prime}$

$2 \quad 5^{\prime}-R\left(U_{1} A_{2} A_{3} G_{4} G_{5} A_{6} G_{7} G_{8} U_{9} G_{10} A_{11} U_{12}\right)-3^{\prime}$

$3 \quad 5^{\prime}-R\left(U_{1} A_{2} A_{3} G_{4} G_{5} A_{6} G_{7} G_{8} U_{9} G_{10} A_{11} U_{12}\right)-3^{\prime}$ 


\section{6. $\quad$ Literature}

[1] Panayides, J.-L.; Mathieu, V.; Banuls, L. M. Y.; Apostolellis, H.; Dahan-Farkas, N.; Davids, H.; Harmse, L.; Rey, M. E. C.; Green, I. R.; Pelly, S. C. et al. Synthesis and in vitro growth inhibitory activity of novel silyl- and trityl-modified nucleosides. Bioorg. Med. Chem. 2016, 24, 2716-2724.

[2] Wunderlich, C. H.; Spitzer, R.; Santner, T.; Fauster, K.; Tollinger, M.; Kreutz, C. Synthesis of $\left(6-{ }^{13} \mathrm{C}\right)$ Pyrimidine Nucleotides as Spin-Labels for RNA Dynamics. J. Am. Chem. Soc. 2012, 134, 7558-7569.

[3] Rameix-Welti, M.-A.; Le Goffic, R.; Hervé, P.-L.; Sourimant, J.; Rémot, A.; Riffault, S.; Yu, Q.; Galloux, M.; Gault, E.; Eléouët, J.-F. Visualizing the replication of respiratory syncytial virus in cells and in living mice. Nat. Commun. 2014, 5, 5104.

[4] Pallan, P. S.; Allerson, C. R.; Berdeja, A.; Seth, P. P.; Swayze, E. E.; Prakash, T. P.; Egli, M. Structure and nuclease resistance of 2',4'-constrained 2'-O-methoxyethyl (cMOE) and 2'-O-ethyl (cEt) modified DNAs. Chem. Commun. 2012, 48, 8195-8197.

[5] Pallan, P. S.; Ittig, D.; Héroux, A.; Wawrzak Z.; Leumann, C. J.; Egli, M. Crystal structure of tricyclo-DNA: an unusal compensatory change of two adjacent backbone torsion angles. Chem. Commun. 2008, 883-885.

[6] Schindelin, H.; Zhang, M.; Bald, R.; Fürste, J.-P.; Erdmann, V. A.; Heinemann, U. J. Mol. Biol. 1995, 249, 595-603. 